\title{
Design, Isolation, and Spectroscopic Analysis of a Tetravalent Terbium Complex
}

\author{
Supporting Information
}

Natalie T. Rice ${ }^{\dagger}$ Ivan A. Popov,${ }^{\ddagger}$ Dominic R. Russo, ${ }^{\dagger}$ John Bacsa,${ }^{\dagger}$ Enrique R. Batista,${ }^{\ddagger}$ Ping Yang,,$\neq$ Joshua Telser,$\$$ and Henry S. La Pierre ${ }^{\dagger, \phi *}$

+ School of Chemistry and Biochemistry, Georgia Institute of Technology, Atlanta, Georgia 30332-0400, United States.

¥ Theoretical Division, Los Alamos National Laboratory, Los Alamos, New Mexico 87545, United States.

$\S$ Department of Biological, Chemical and Physical Sciences, Roosevelt University, Chicago, Illinois 60605, United States.

$\phi$ Nuclear and Radiological Engineering and Medical Physics Program, School of Mechanical Engineering, Georgia Institute of Technology, Atlanta, Georgia 30332-0400, United States

\section{Table of Contents}

NMR Spectra of Reported Compounds .................................................................. 3

Crystallographic Information ............................................................................ 13

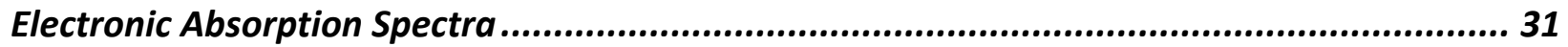

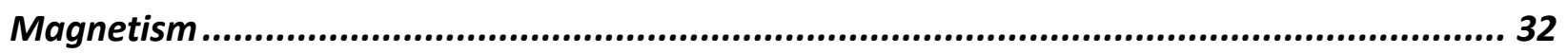

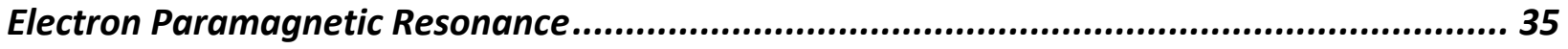

Theoretical Details................................................................................................ 36

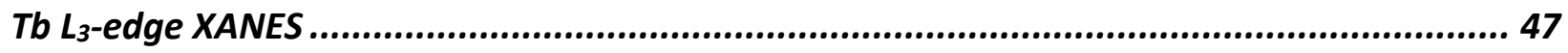

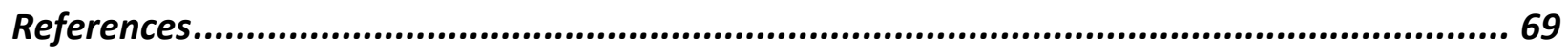


A

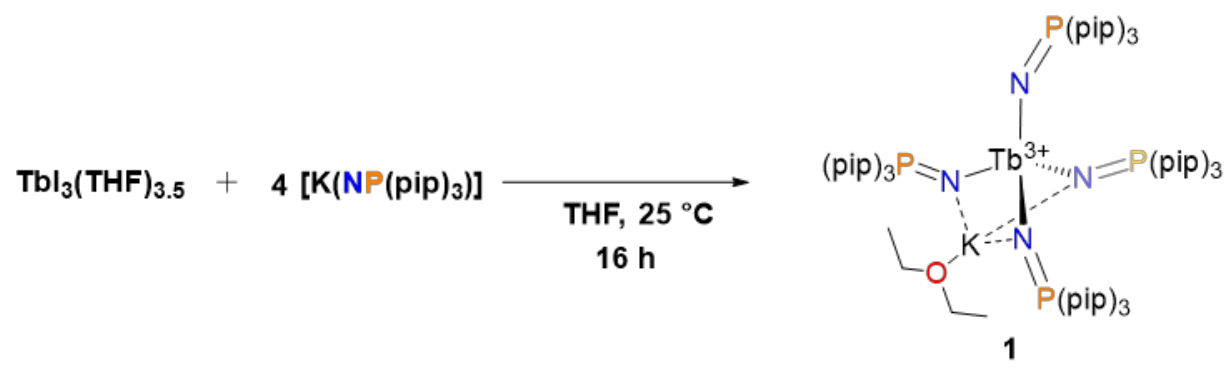

B
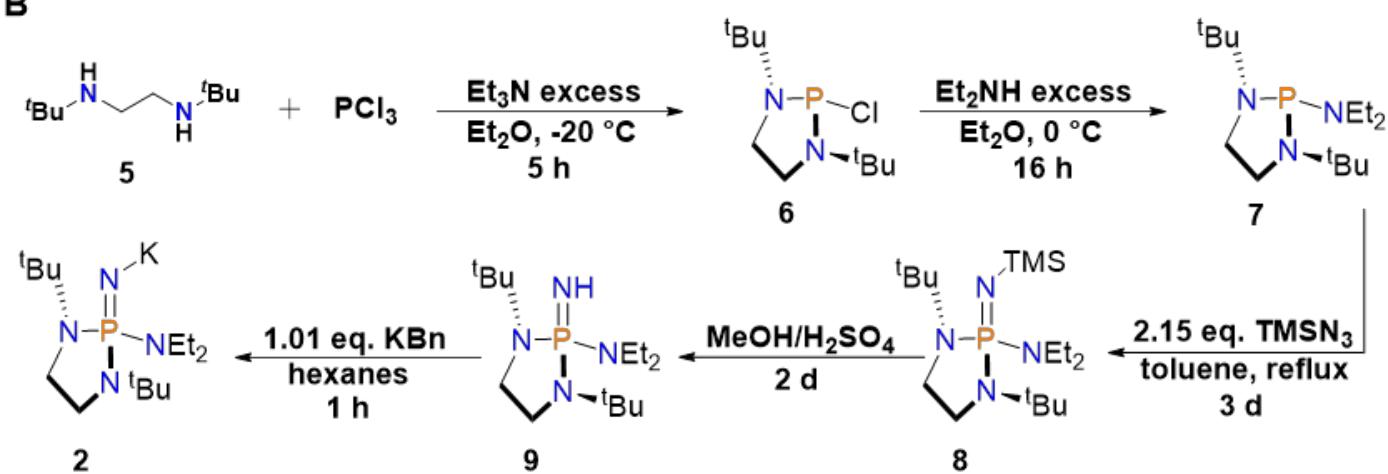

C

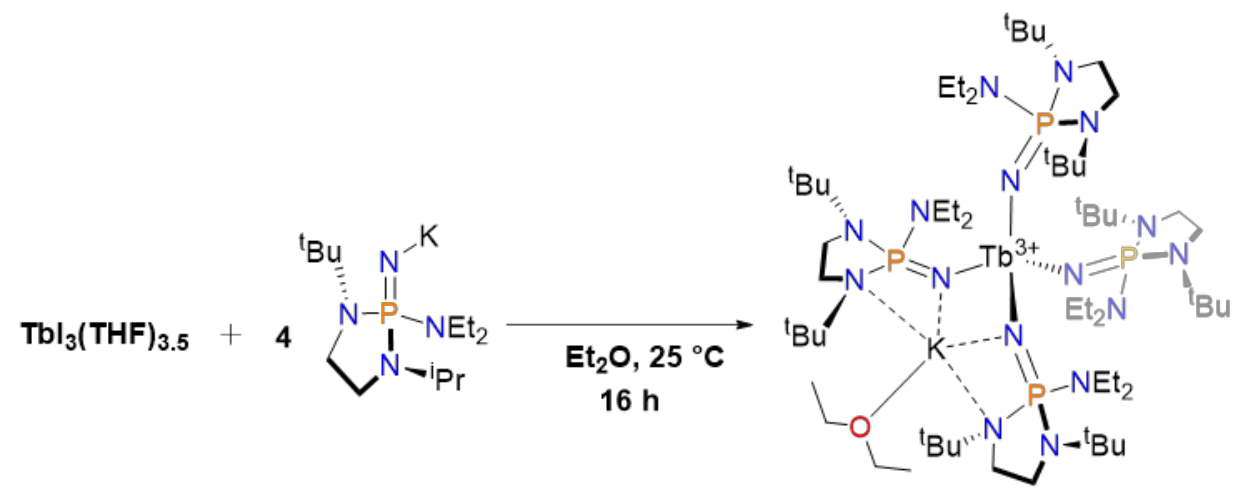

3

Figure S1. General synthetic route of the reported compounds. 
NMR Spectra of Reported Compounds

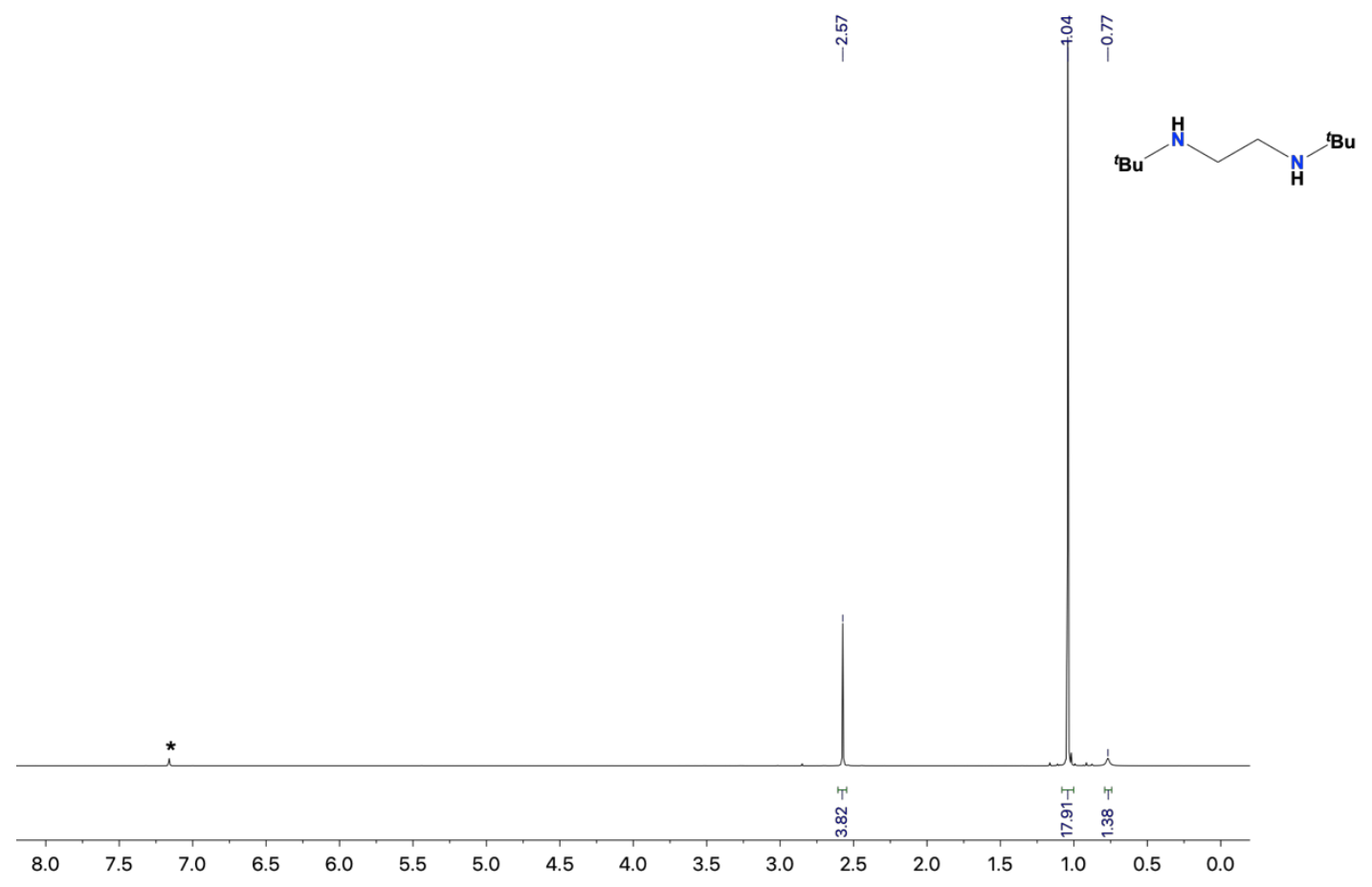

Figure S2. ${ }^{1} \mathrm{H}$ NMR of 5 in $\mathrm{C}_{6} \mathrm{D}_{6}$. Peak of $\mathrm{C}_{6} \mathrm{D}_{5} \mathrm{H}$ is noted as *.

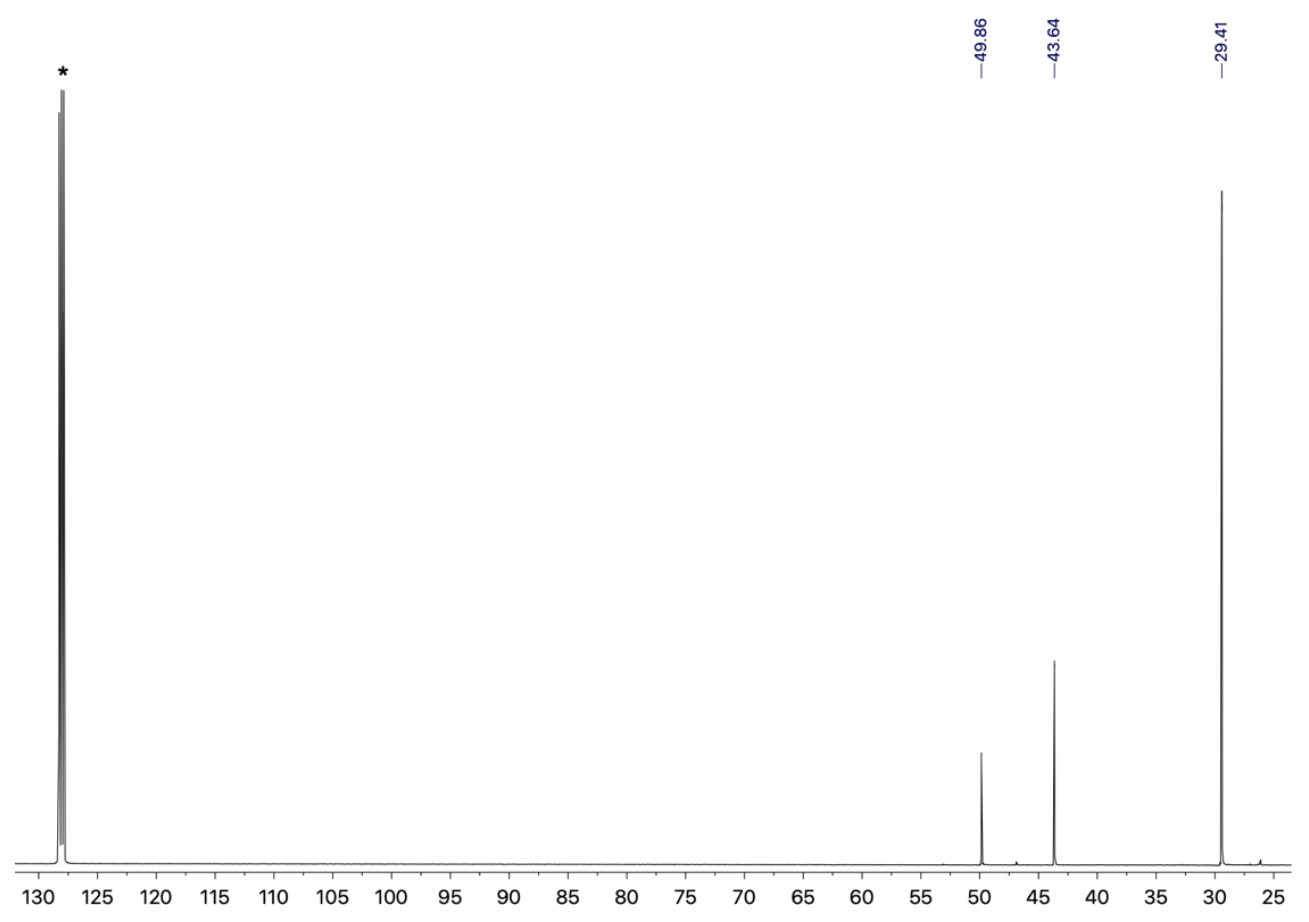

Figure S3. ${ }^{13} \mathrm{C}\left\{{ }^{1} \mathrm{H}\right\}$ NMR of 5 in $\mathrm{C}_{6} \mathrm{D}_{6}$. Peak of $\mathrm{C}_{6} \mathrm{D}_{5} \mathrm{H}$ is noted as *. 


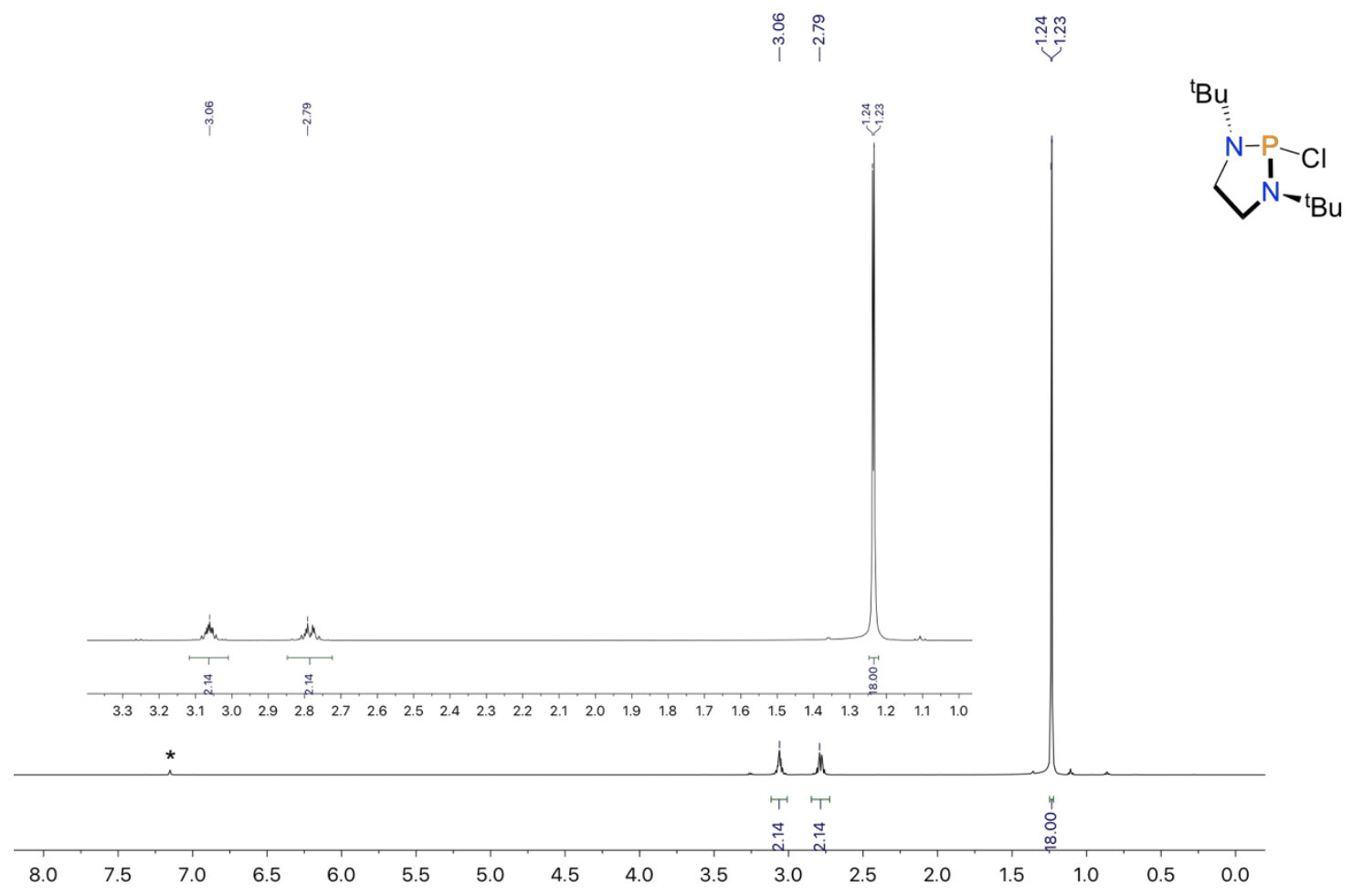

Figure S4. ${ }^{1} \mathrm{H}$ NMR of 6 in $\mathrm{C}_{6} \mathrm{D}_{6}$. Peak of $\mathrm{C}_{6} \mathrm{D}_{5} \mathrm{H}$ is noted as *.

Figure S5. ${ }^{31} \mathrm{P}\left\{{ }^{1} \mathrm{H}\right\}$ NMR of 6 in $\mathrm{C}_{6} \mathrm{D}_{6}$. 


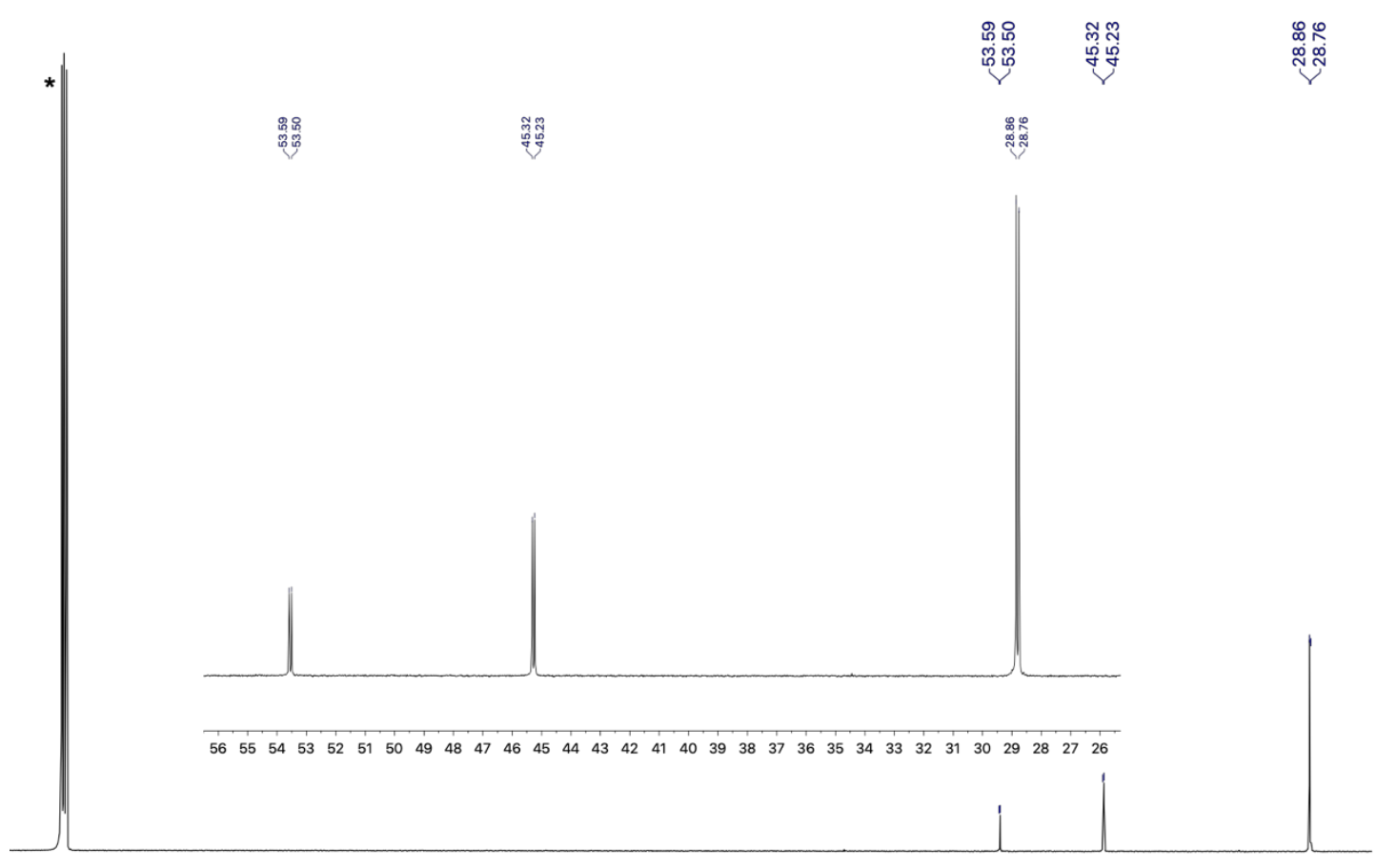

$\begin{array}{llllllllllllllllllllllllll}130 & 125 & 120 & 115 & 110 & 105 & 100 & 95 & 90 & 85 & 80 & 75 & 70 & 65 & 60 & 55 & 50 & 45 & 40 & 35 & 30 & 25\end{array}$

Figure S6. ${ }^{13} \mathrm{C}\left\{{ }^{1} \mathrm{H}\right\}$ NMR of 6 in $\mathrm{C}_{6} \mathrm{D}_{6}$. Peak of $\mathrm{C}_{6} \mathrm{D}_{5} \mathrm{H}$ is noted as *.

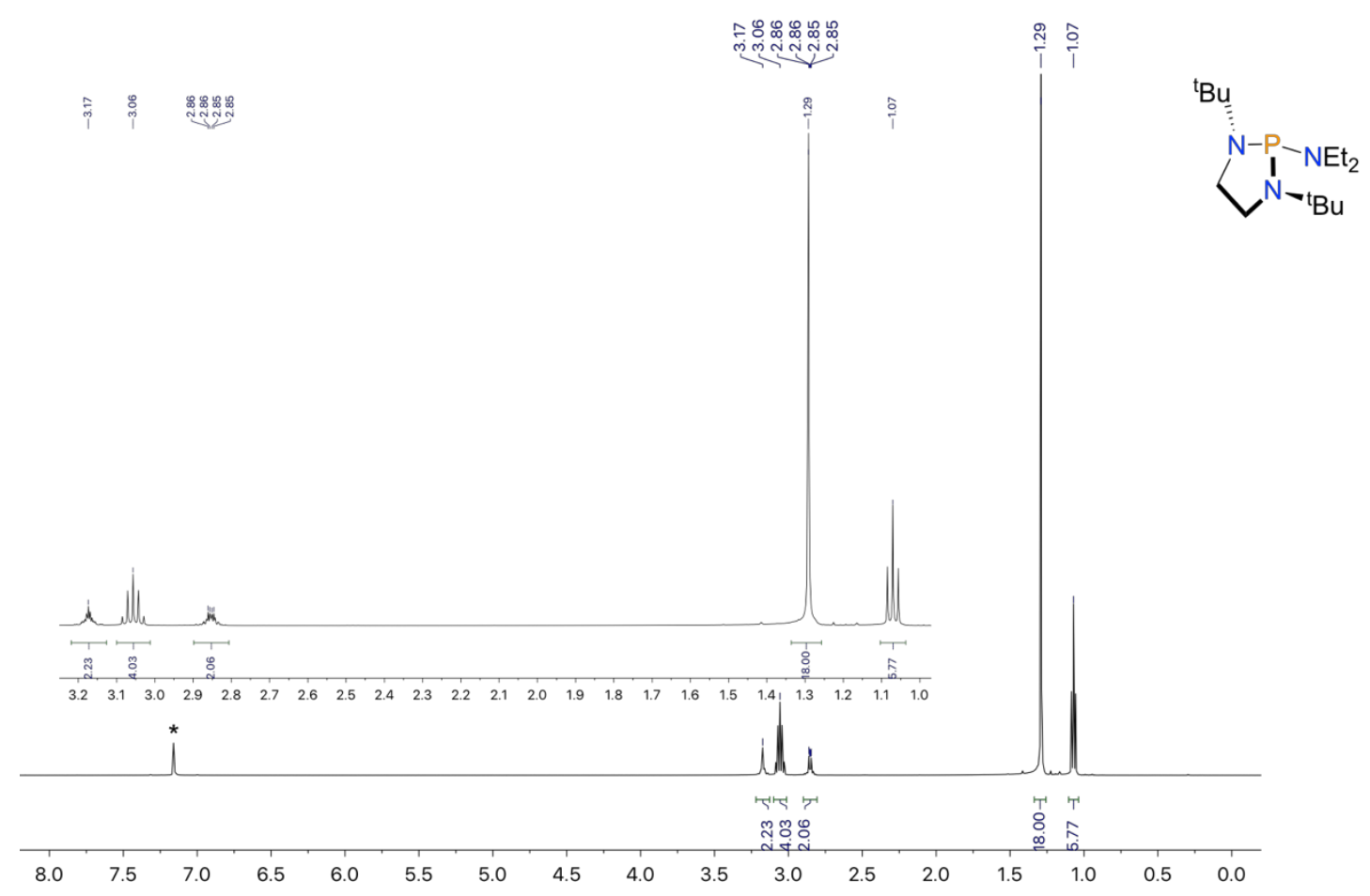

Figure S7. ${ }^{1} \mathrm{H}$ NMR of 7 in $\mathrm{C}_{6} \mathrm{D}_{6}$. Peak of $\mathrm{C}_{6} \mathrm{D}_{5} \mathrm{H}$ is noted as *. 


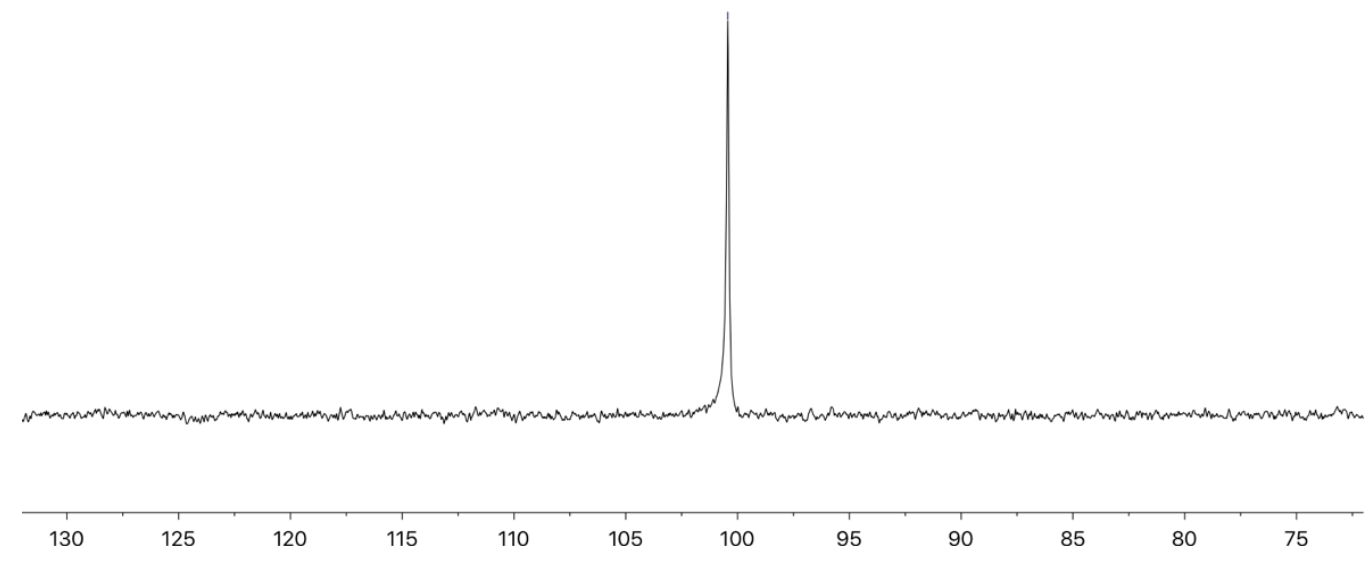

Figure S8. ${ }^{31} \mathrm{P}\left\{{ }^{1} \mathrm{H}\right\} \mathrm{NMR}$ of 7 in $\mathrm{C}_{6} \mathrm{D}_{6}$.

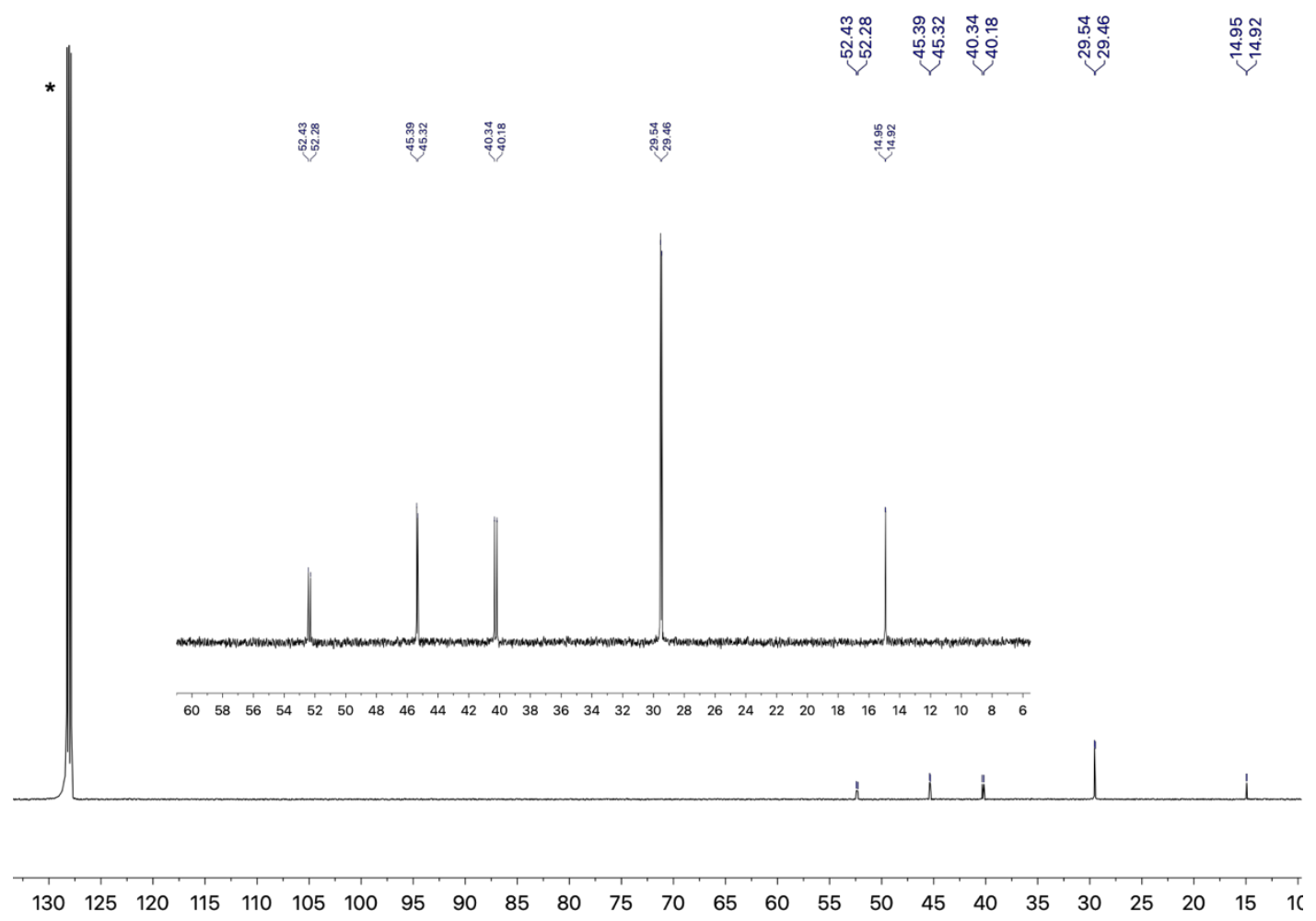

Figure S9. ${ }^{13} \mathrm{C}\left\{{ }^{1} \mathrm{H}\right\}$ NMR of 7 in $\mathrm{C}_{6} \mathrm{D}_{6}$. Peak of $\mathrm{C}_{6} \mathrm{D}_{5} \mathrm{H}$ is noted as *. 


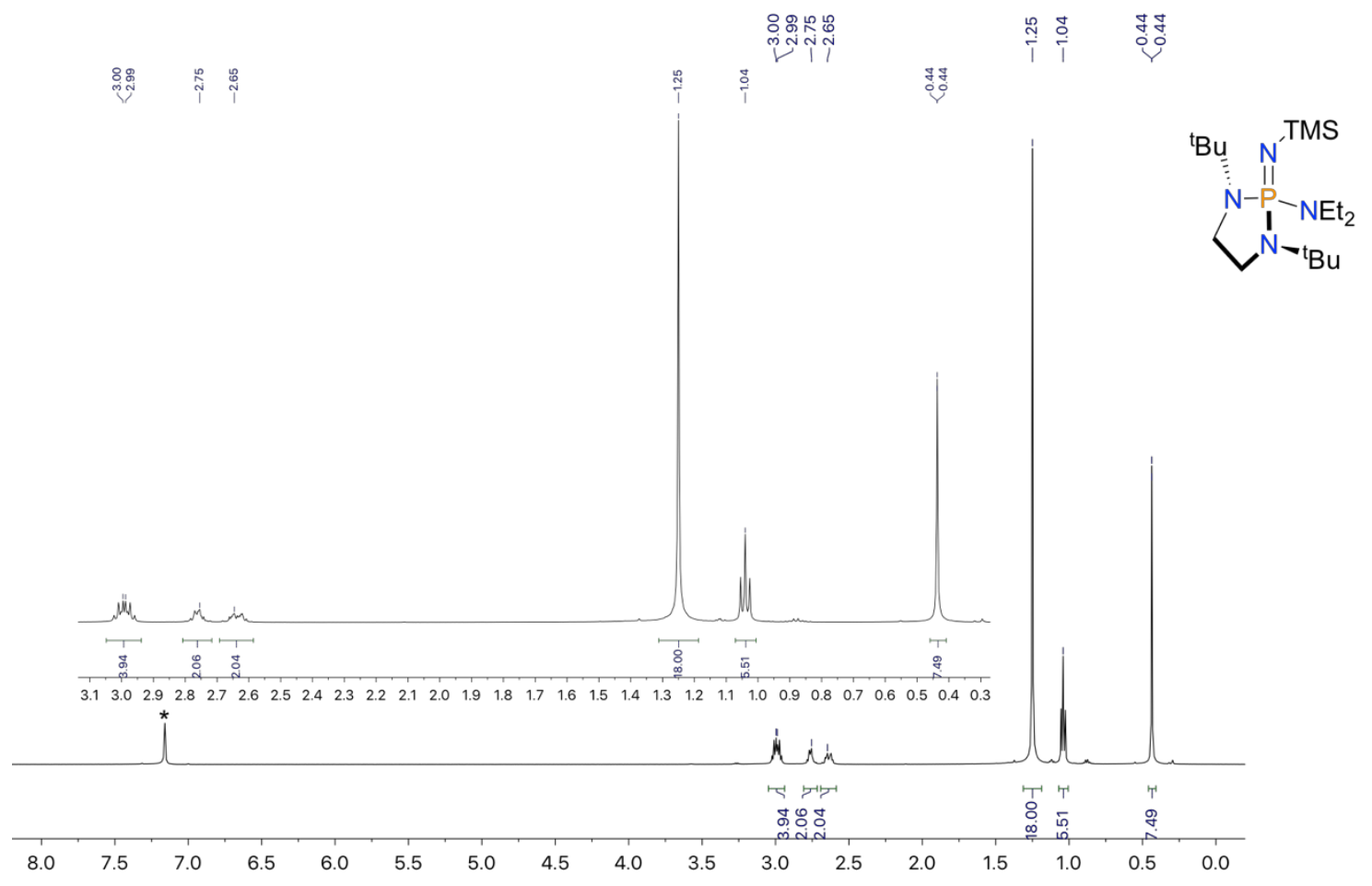

Figure S10. ${ }^{1} \mathrm{H}$ NMR of $\left[\mathrm{TMSN}=\mathrm{P}\left(1,2-\right.\right.$ bis- $^{t} \mathrm{Bu}$-diamidoethane $\left.)\left(\mathrm{NEt}_{2}\right)\right]$ in $\mathrm{C}_{6} \mathrm{D}_{6}$. Peak of $\mathrm{C}_{6} \mathrm{D}_{5} \mathrm{H}$ is noted as *.

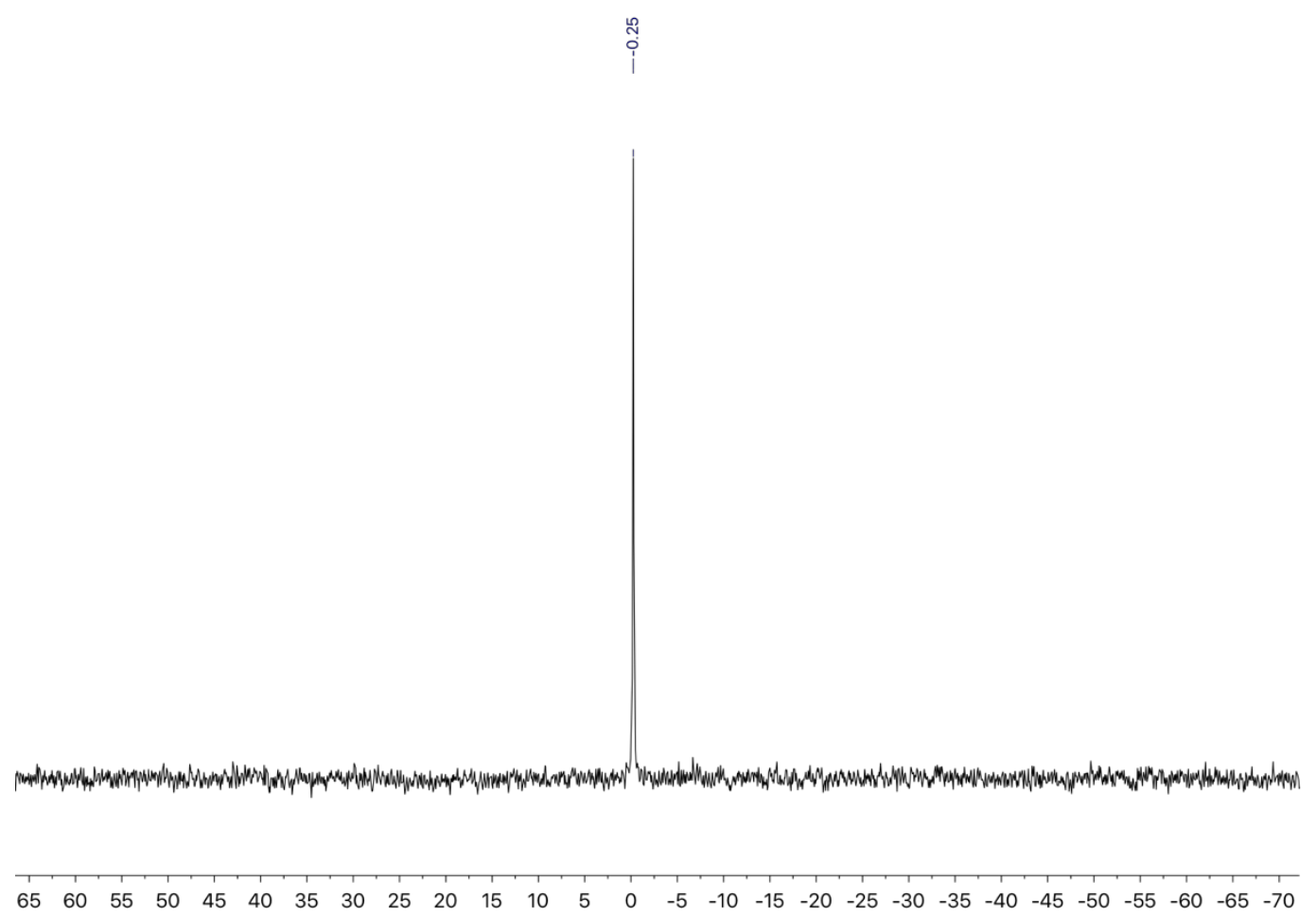

Figure S11. ${ }^{31} \mathrm{P}\left\{{ }^{1} \mathrm{H}\right\}$ NMR of $\left[\mathrm{TMSN}=\mathrm{P}\left(1,2-\right.\right.$ bis- $^{t} \mathrm{Bu}$-diamidoethane $\left.)\left(\mathrm{NEt}_{2}\right)\right]$ in $\mathrm{C}_{6} \mathrm{D}_{6}$. 


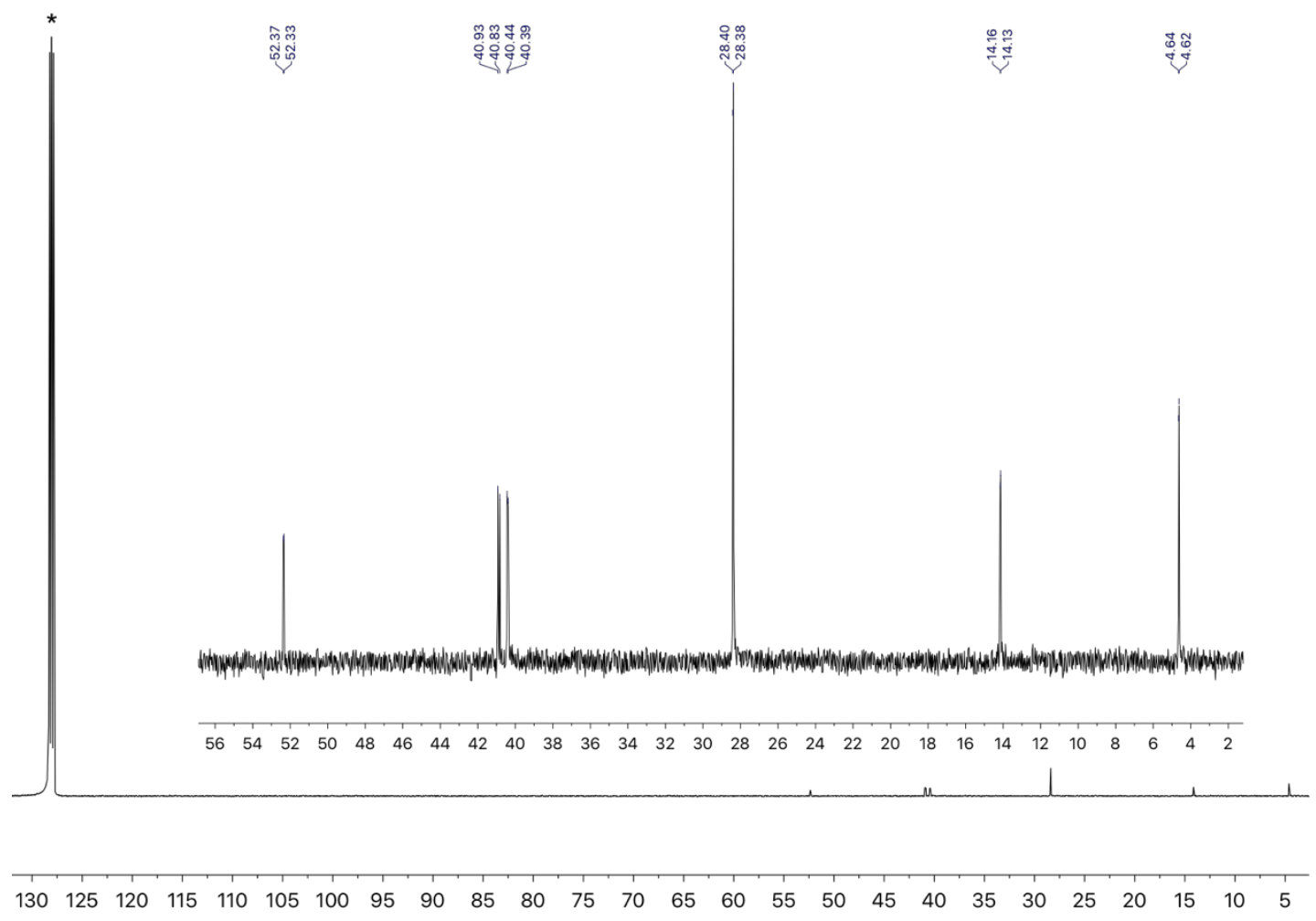

Figure S12. ${ }^{13} \mathrm{C}\left\{{ }^{1} \mathrm{H}\right\}$ NMR of $\left[\mathrm{TMSN}=\mathrm{P}\left(1,2-b i s-{ }^{t} \mathrm{Bu}\right.\right.$-diamidoethane $\left.)\left(\mathrm{NEt}_{2}\right)\right]$ in $\mathrm{C}_{6} \mathrm{D}_{6}$. Peak of $\mathrm{C}_{6} \mathrm{D}_{5} \mathrm{H}$ is noted as *.

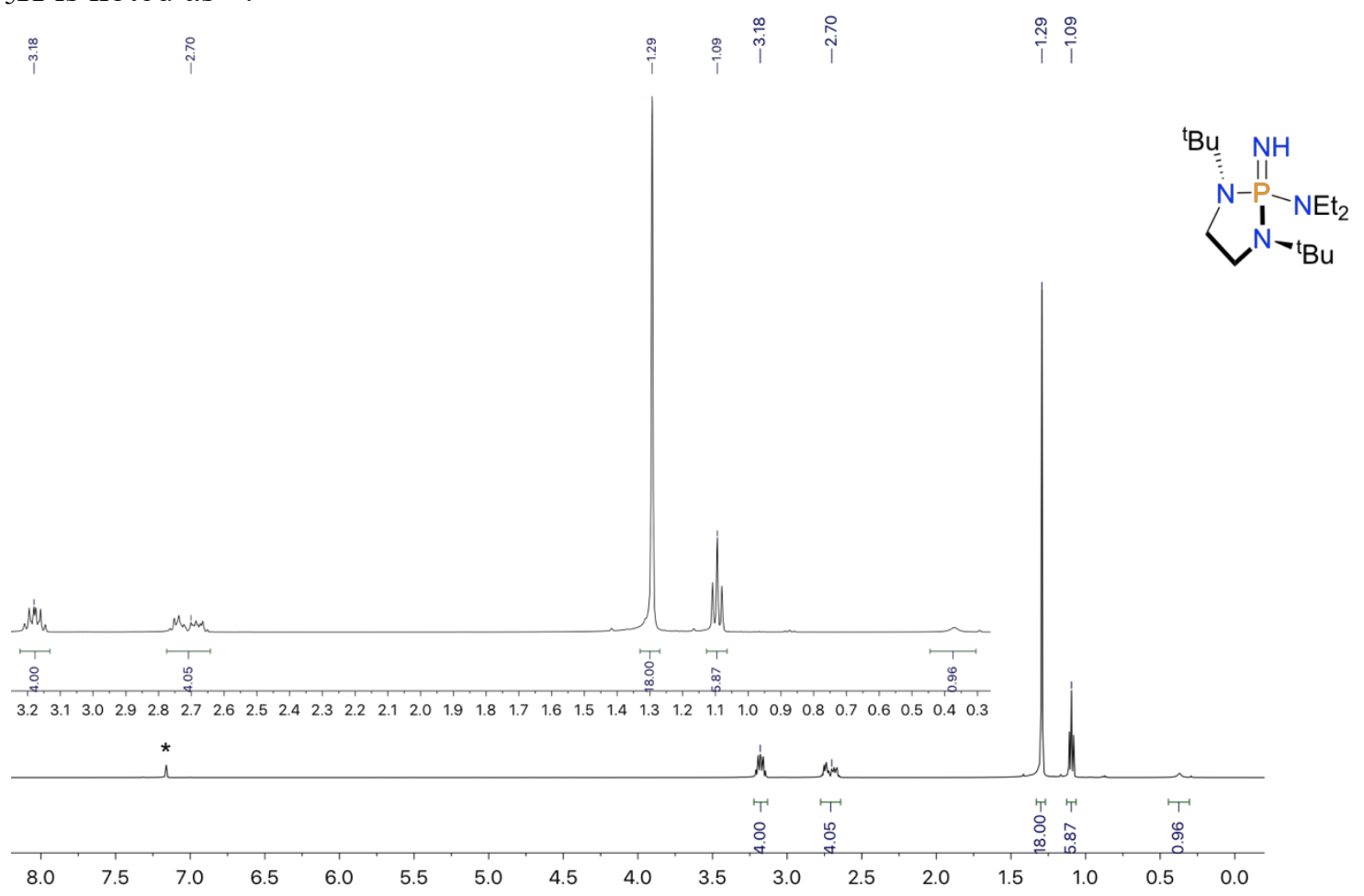

Figure S13. ${ }^{1} \mathrm{H}$ NMR of 8 in $\mathrm{C}_{6} \mathrm{D}_{6}$. Peak of $\mathrm{C}_{6} \mathrm{D}_{5} \mathrm{H}$ is noted as *. 


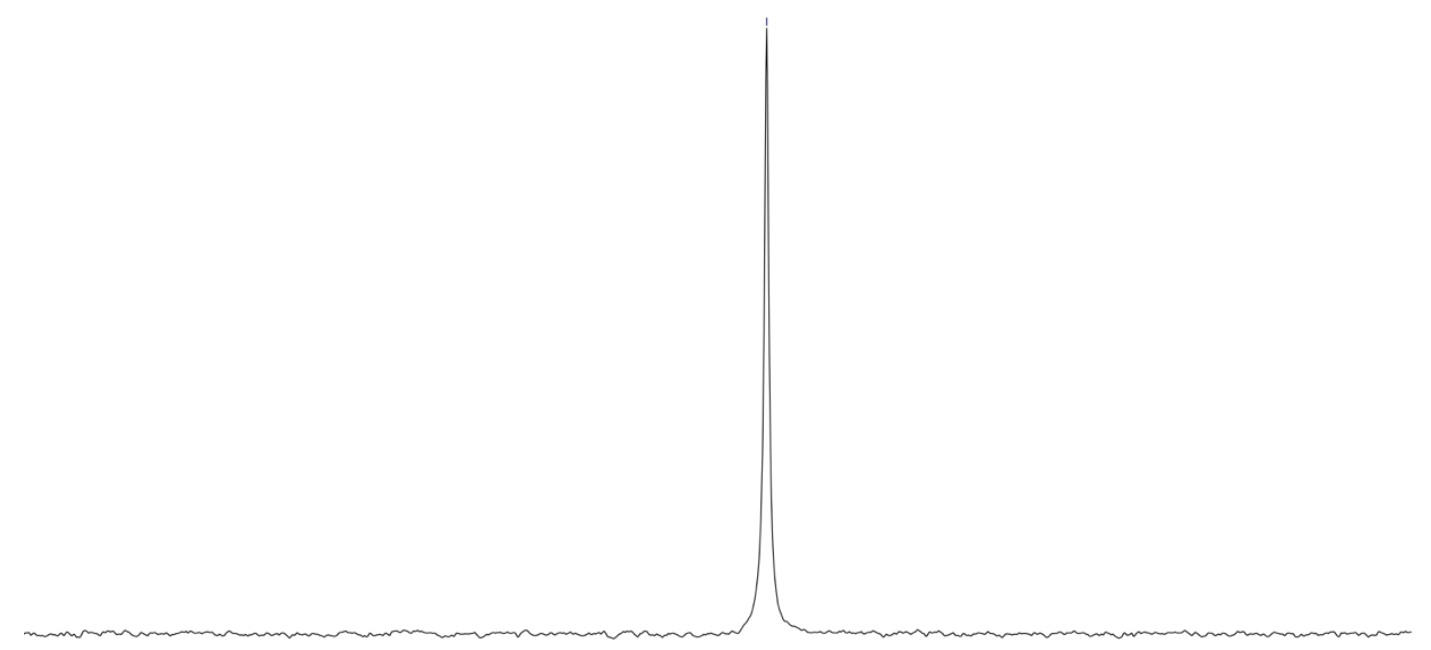

$\begin{array}{lllllllllllllllllllllllllllll}42 & 41 & 40 & 39 & 38 & 37 & 36 & 35 & 34 & 33 & 32 & 31 & 30 & 29 & 28 & 27 & 26 & 25 & 24 & 23 & 22 & 21\end{array}$

Figure S14. ${ }^{31} \mathrm{P}\left\{{ }^{1} \mathrm{H}\right\} \mathrm{NMR}$ of 8 in $\mathrm{C}_{6} \mathrm{D}_{6}$.

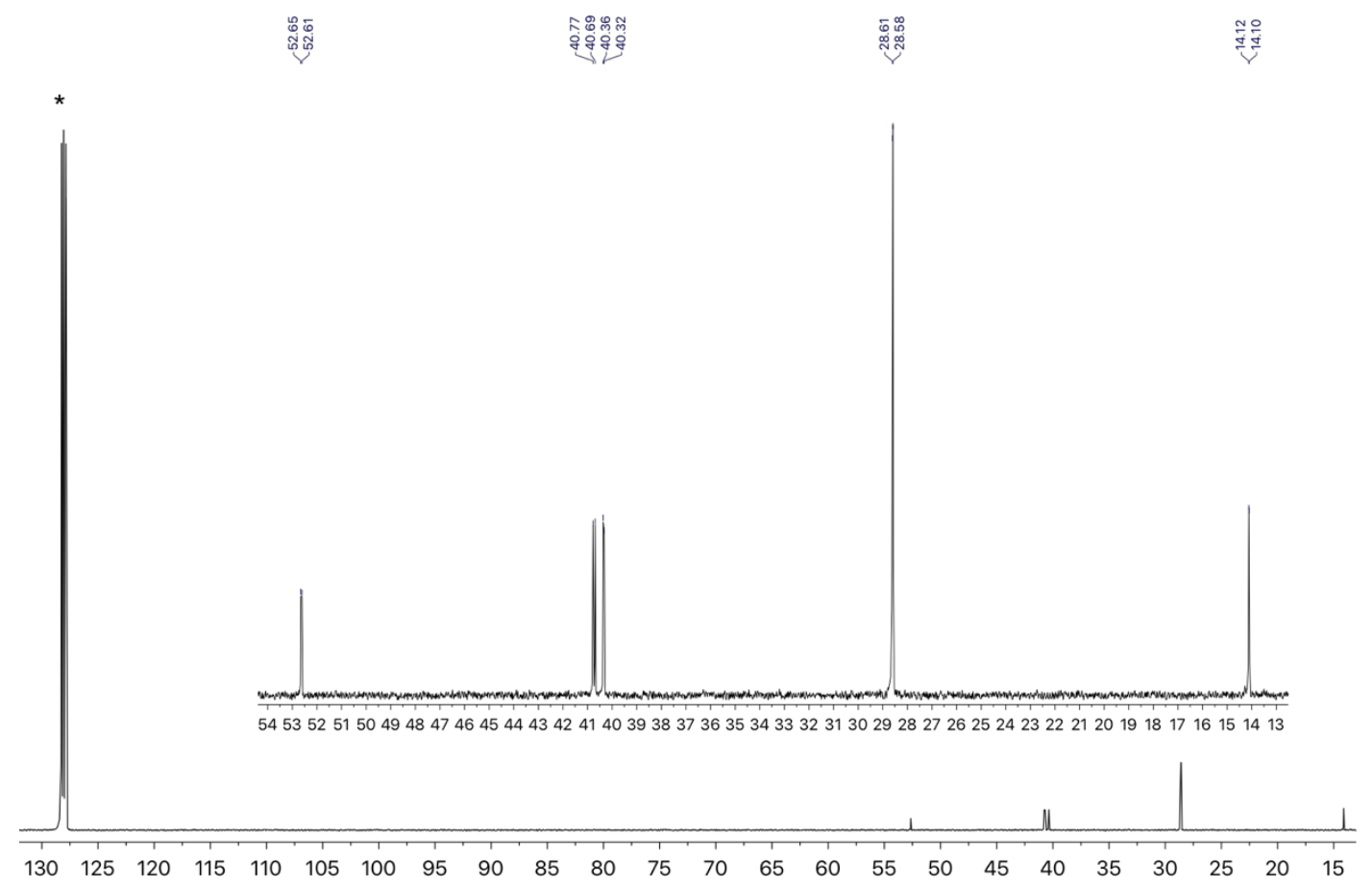

Figure S15. ${ }^{13} \mathrm{C}\left\{{ }^{1} \mathrm{H}\right\}$ NMR of 8 in $\mathrm{C}_{6} \mathrm{D}_{6}$. Peak of $\mathrm{C}_{6} \mathrm{D}_{5} \mathrm{H}$ is noted as *. 


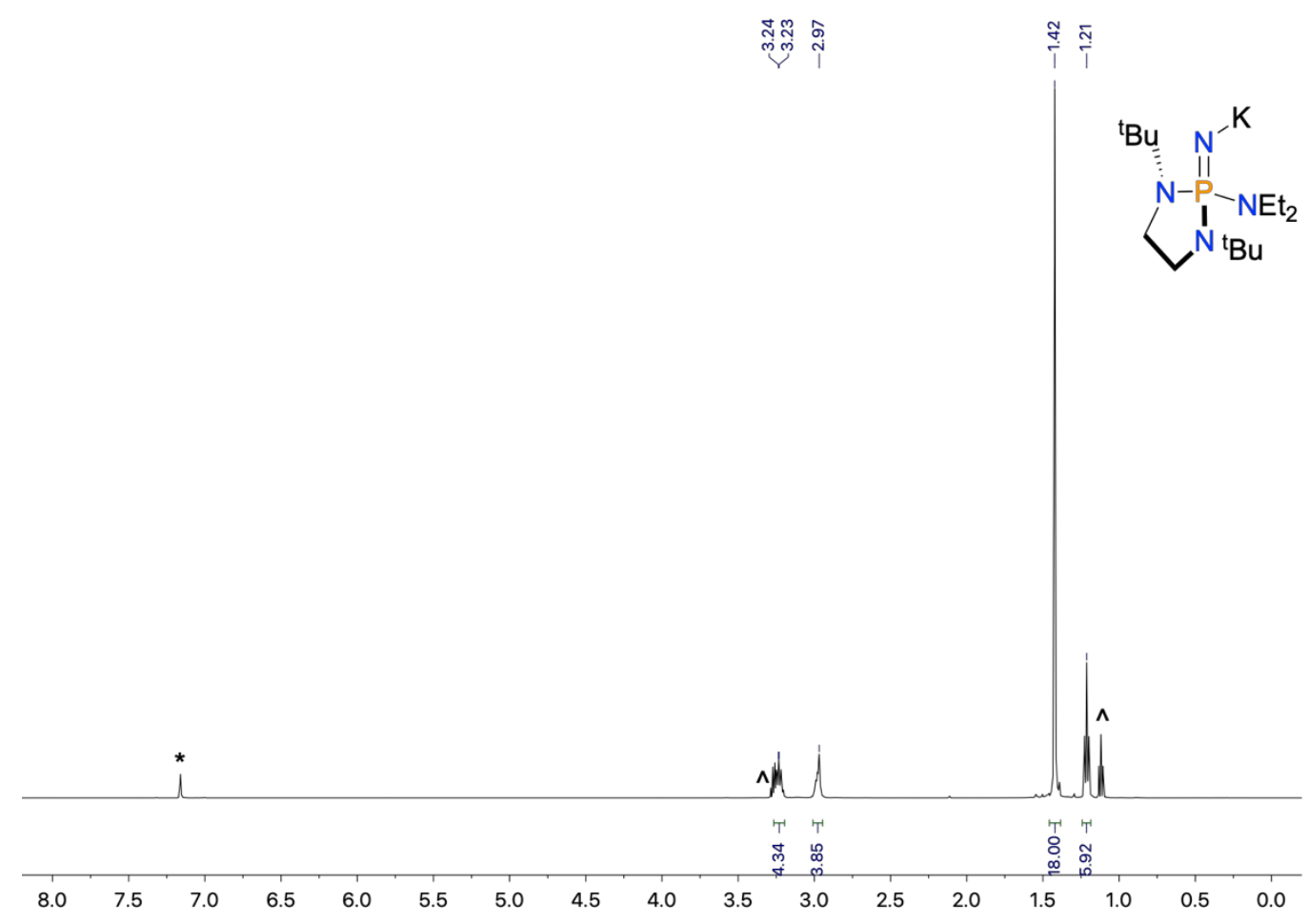

Figure S16. ${ }^{1} \mathrm{H}$ NMR of 2 in $\mathrm{C}_{6} \mathrm{D}_{6}$. Peak of $\mathrm{C}_{6} \mathrm{D}_{5} \mathrm{H}$ is noted as *. Residual diethyl ether is denoted as $\wedge$.

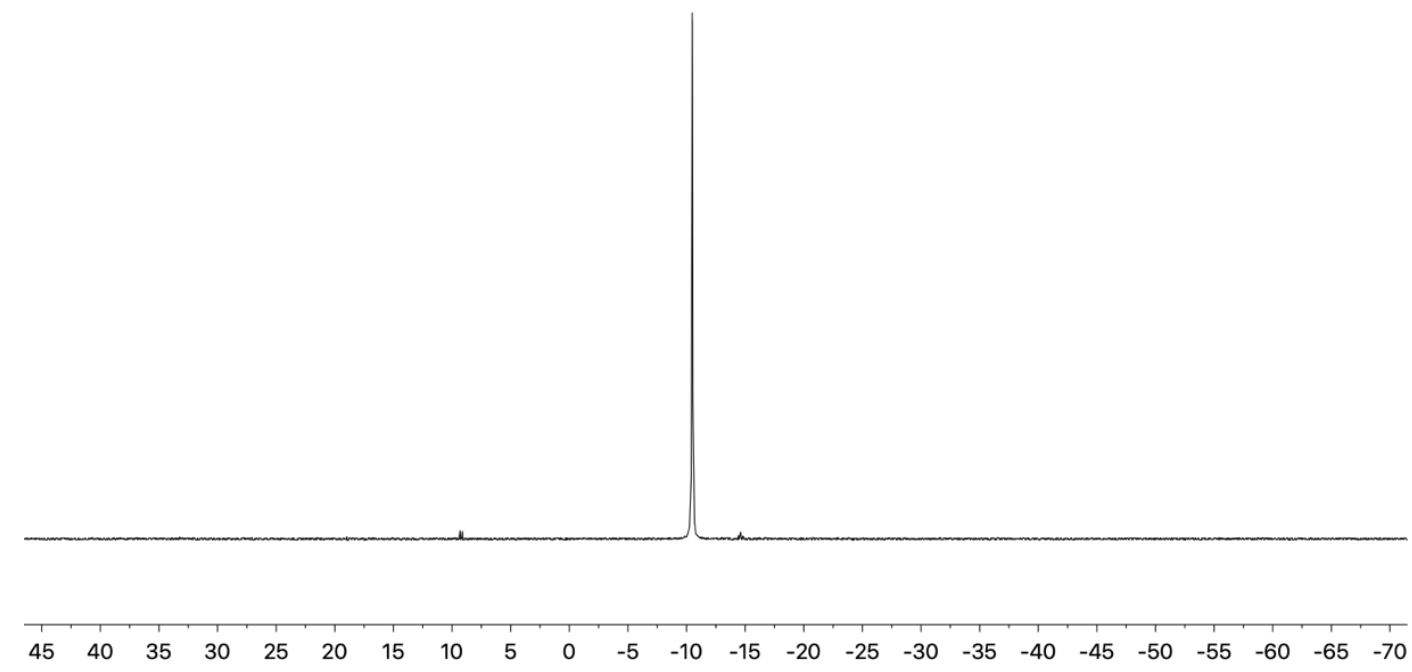

Figure S17. ${ }^{31} \mathrm{P}\left\{{ }^{1} \mathrm{H}\right\}$ NMR of 2 in $\mathrm{C}_{6} \mathrm{D}_{6}$. 


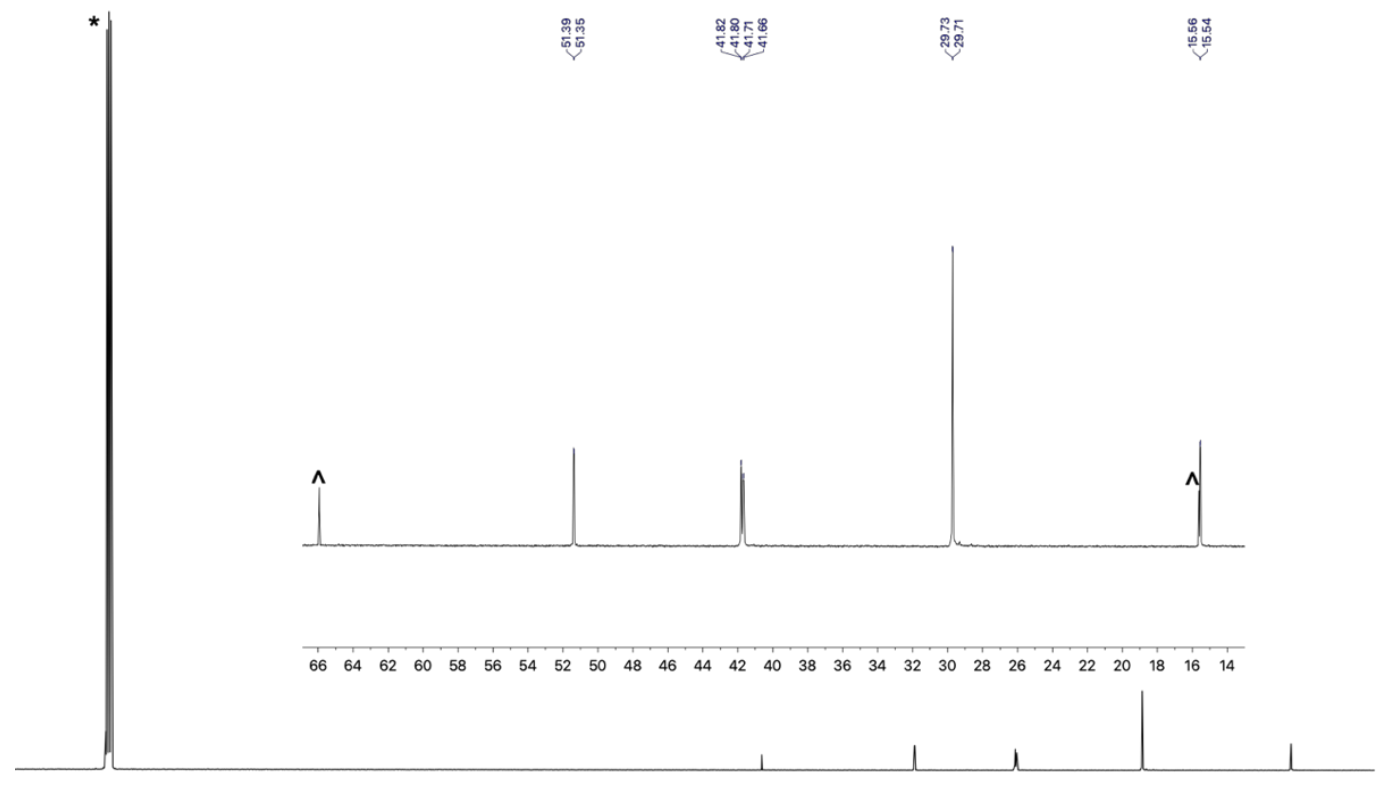

$\begin{array}{llllllllllllllllllllllllllllllll}135 & 130 & 125 & 120 & 115 & 110 & 105 & 100 & 95 & 90 & 85 & 80 & 75 & 70 & 65 & 60 & 55 & 50 & 45 & 40 & 35 & 30 & 25 & 20 & 15 & 10\end{array}$

Figure S18. ${ }^{13} \mathrm{C}\left\{{ }^{1} \mathrm{H}\right\}$ NMR of 2 in $\mathrm{C}_{6} \mathrm{D}_{6}$. Peak of $\mathrm{C}_{6} \mathrm{D}_{5} \mathrm{H}$ is noted as *. Residual diethyl ether is denoted as ${ }^{\wedge}$.

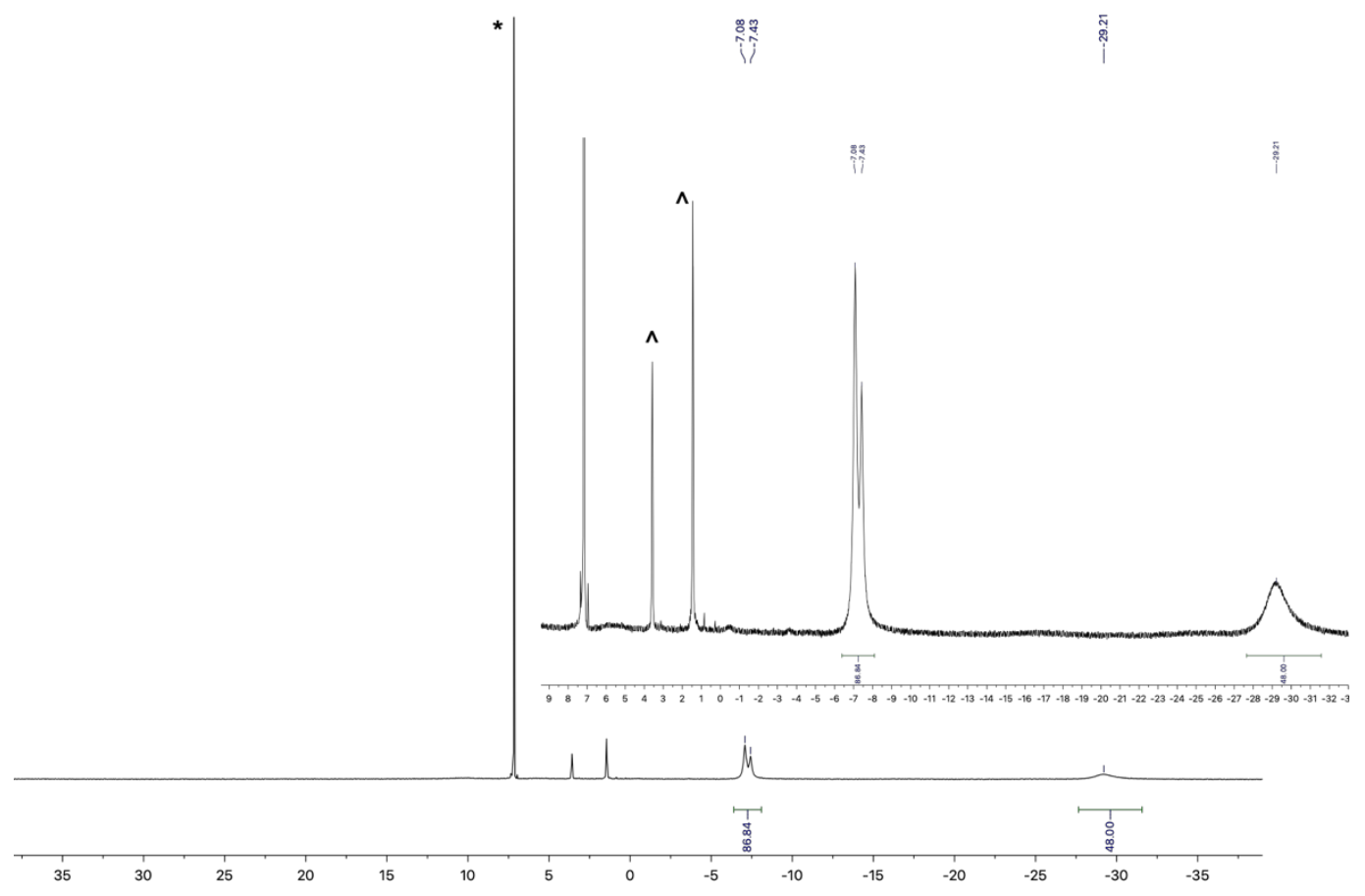

Figure S19. ${ }^{1} \mathrm{H}$ NMR of 1 in $\mathrm{C}_{6} \mathrm{D}_{6}$. Peak of $\mathrm{C}_{6} \mathrm{D}_{5} \mathrm{H}$ is noted as *. Residual THF is denoted as ${ }^{\wedge}$. 


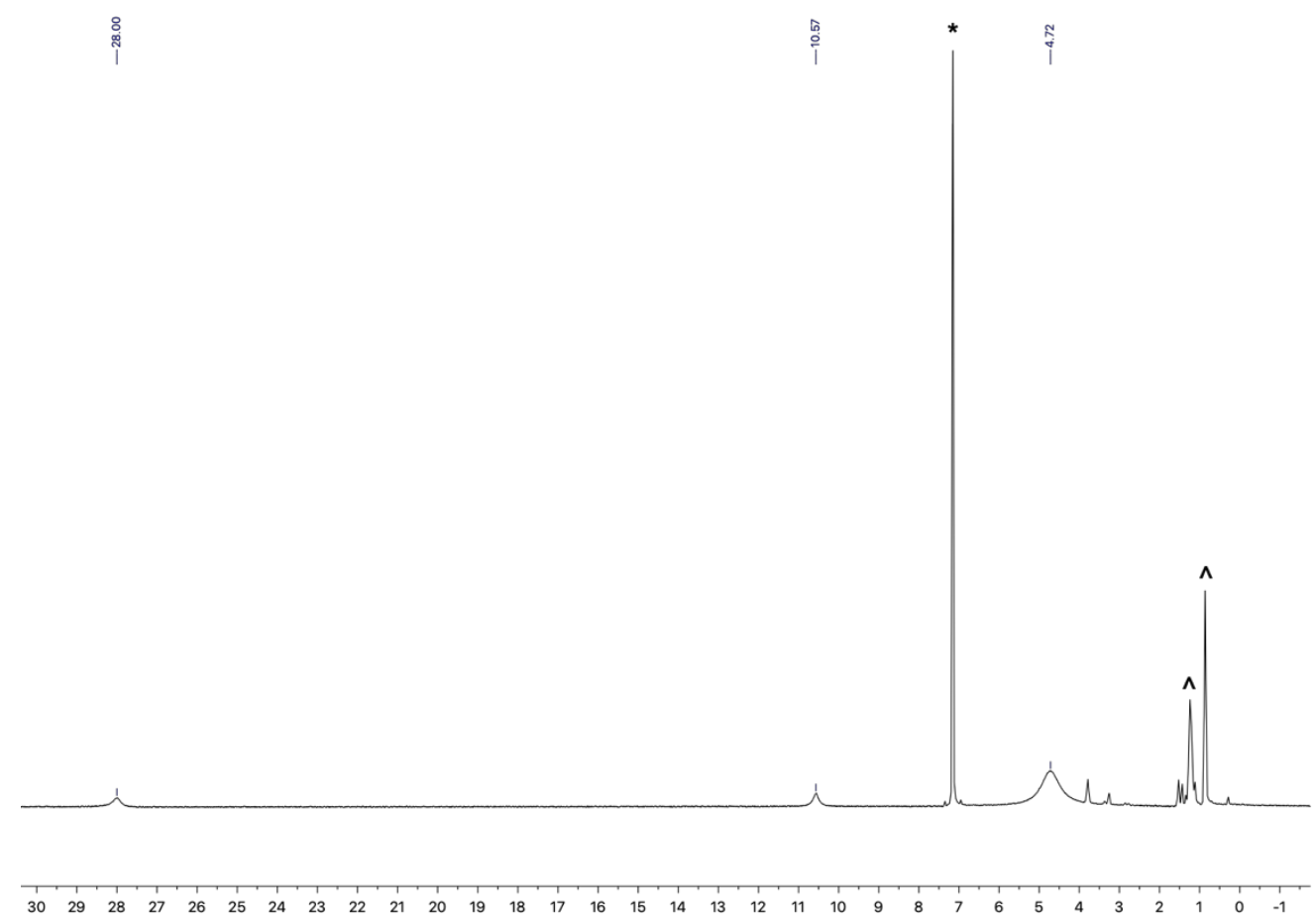

Figure S20. ${ }^{1} \mathrm{H}$ NMR of 3 in $\mathrm{C}_{6} \mathrm{D}_{6}$. Peak of $\mathrm{C}_{6} \mathrm{D}_{5} \mathrm{H}$ is noted as *. Residual $n$-pentane is denoted as ${ }^{\wedge}$. 


\section{Crystallographic Information}

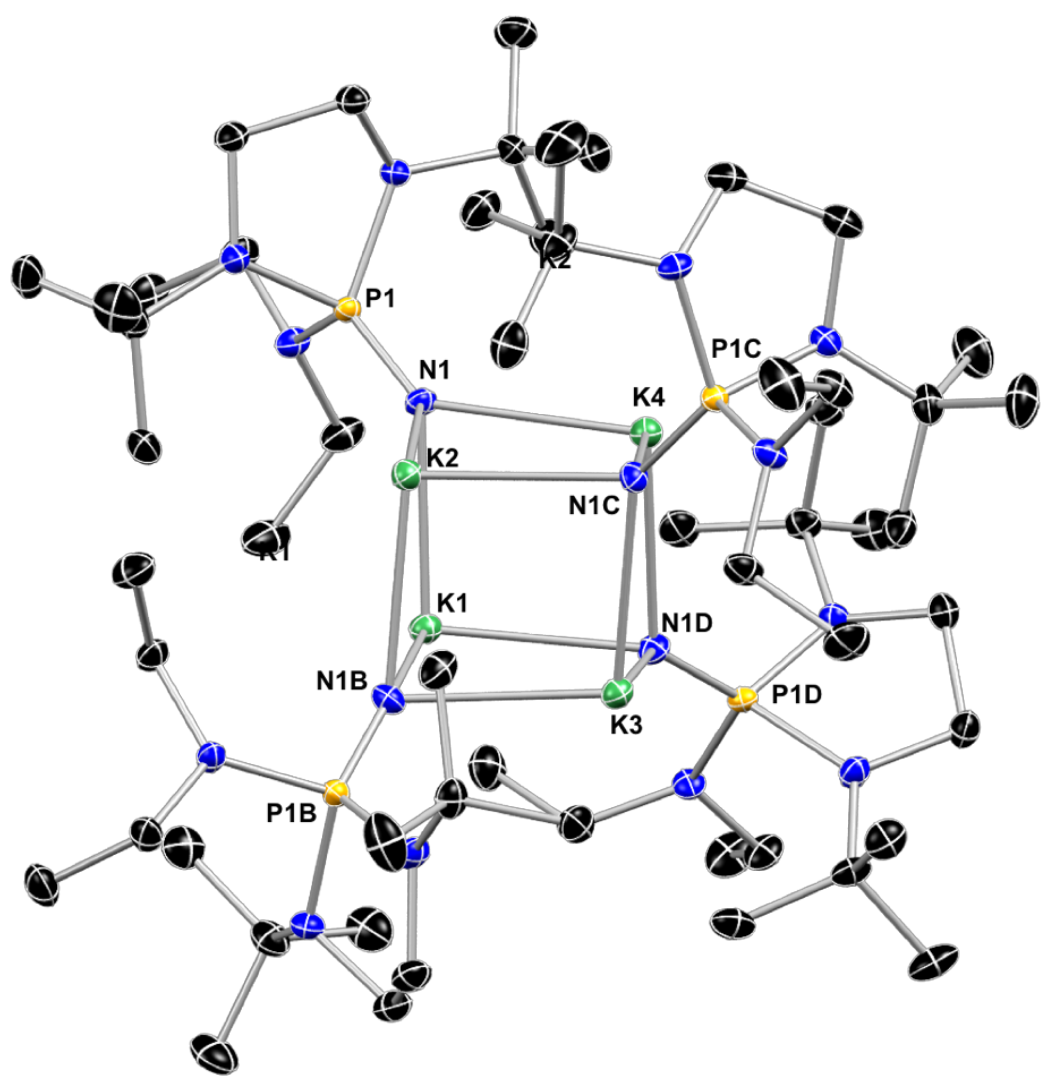

Figure S21. Molecular structure of 2 at with thermal ellipsoids shown at 50\% probability. H atoms and co-crystallized diethyl ether molecule are omitted for clarity. 


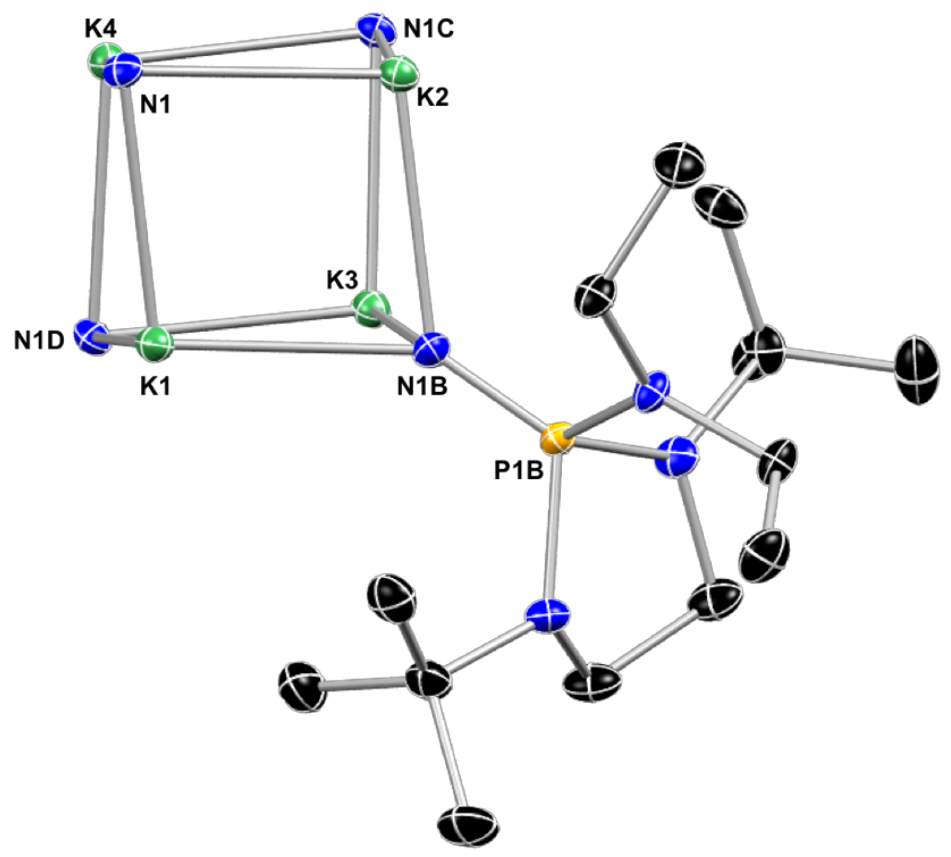

Figure S22. Molecular structure of 2 at with thermal ellipsoids shown at 50\% probability. H atoms, co-crystallized diethyl ether molecule, and all but one ligand arm are omitted for clarity.

Table S1. Comparison of crystallographic metrics for 2 and $\left[\mathrm{PN}(\mathrm{pip})_{3}\right] \mathrm{K}$.

\begin{tabular}{|c|c|c|}
\hline Metric Average & {$\left[\mathrm{PN}(\text { pip) })_{3}\right] \mathrm{K}$} & 2 \\
\hline $\mathbf{N}_{\text {imide }}-\mathbf{P}(\AA)$ & $1.519(4)$ & $1.522(1)$ \\
\hline P-N $\mathbf{N}_{\text {amido }}(\AA)$ & $1.707(4)$ & $1.703(1)$ \\
\hline P-N diamido $(\AA)$ & - & $1.722(1)$ \\
\hline K-N imide $(\AA ̊)$ & $2.728(4)$ & $2.714(1)$ \\
\hline N-P-N ${ }_{\text {amido }}\left({ }^{\circ}\right)$ & $116.8(2)$ & $113.76(5)$ \\
\hline N-P-N $\mathbf{d i a m i d o}\left({ }^{\circ}\right)$ & - & $121.00(4)$ \\
\hline
\end{tabular}




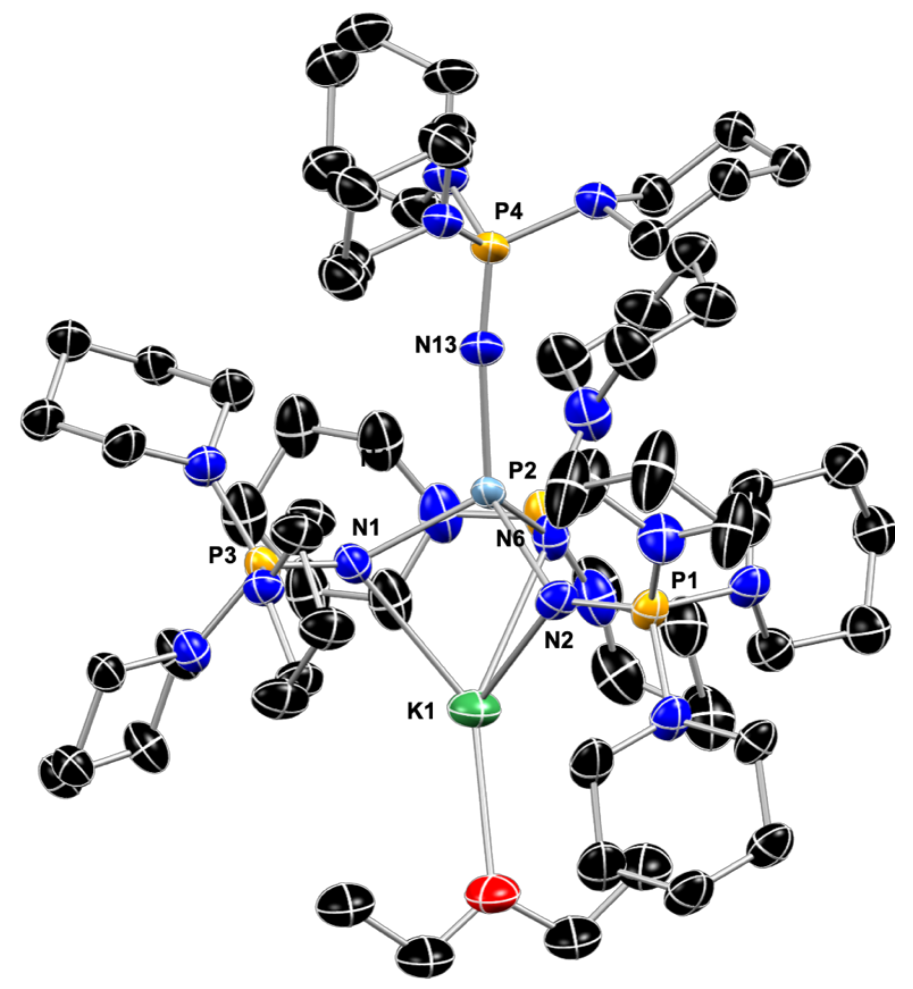

Figure S23. Molecular structure of 1 with thermal ellipsoids shown at 50\% probability. H atoms are omitted for clarity.

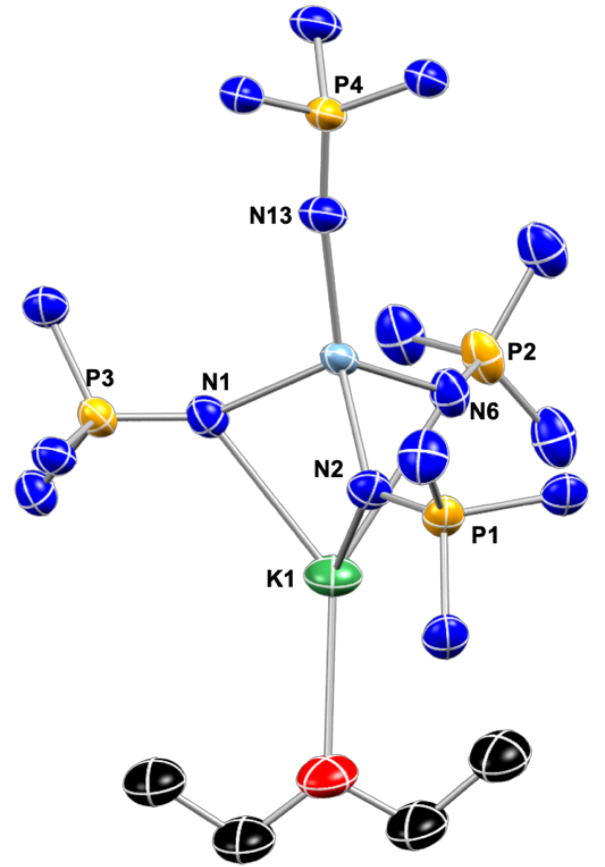

Figure S24. Molecular structure of 1 with thermal ellipsoids shown at 50\% probability. H atoms and carbon atoms of the ligand are omitted for clarity. 


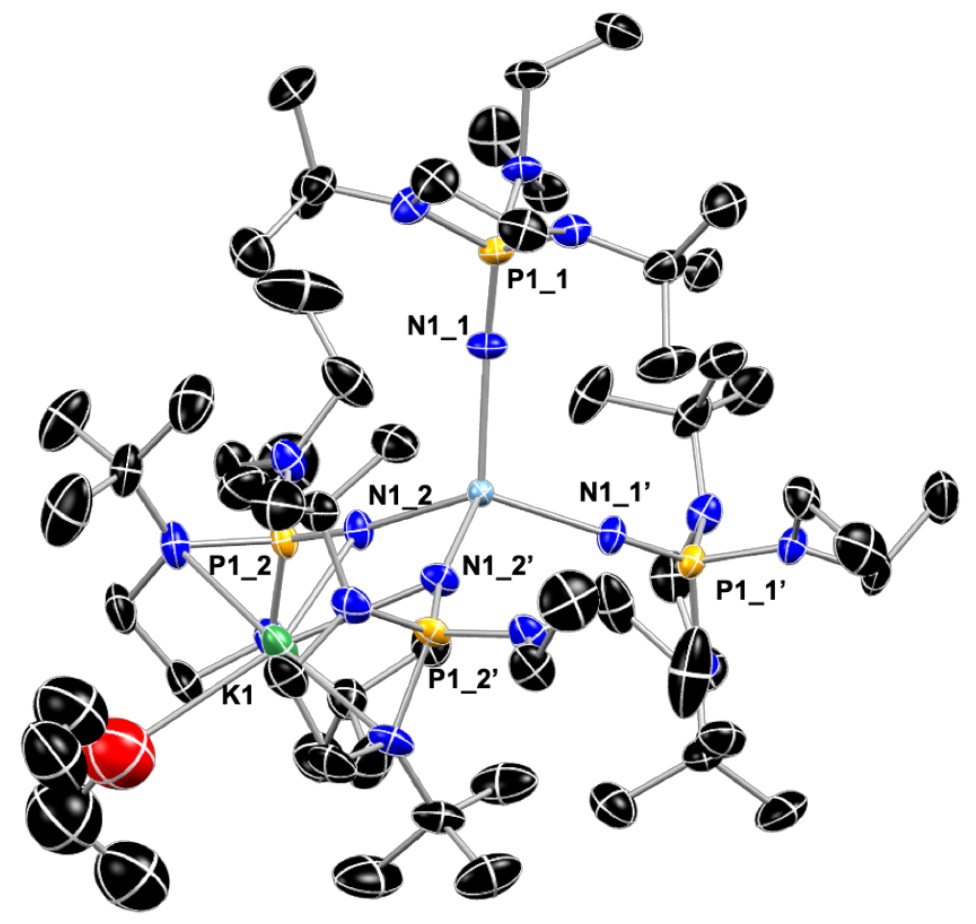

Figure S25. Molecular structure of 3 with thermal ellipsoids shown at 50\% probability. H atoms are omitted for clarity.

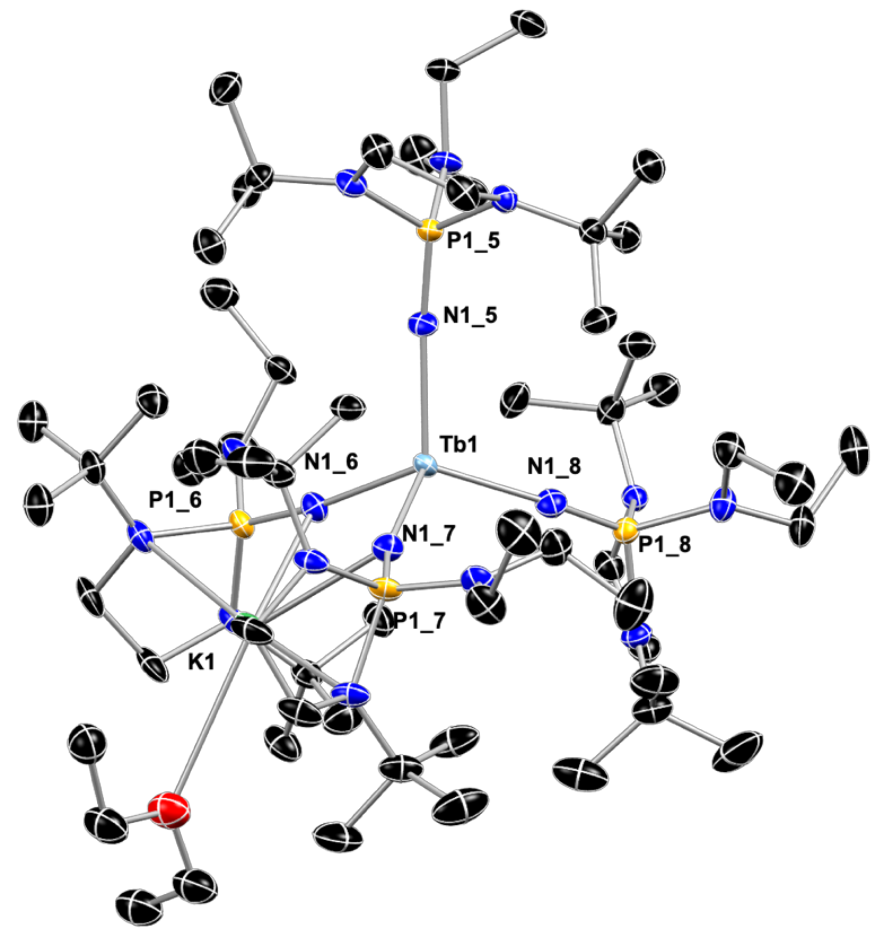

Figure S26. Molecular structure of 3-100K with thermal ellipsoids shown at 50\% probability. $\mathrm{H}$ atoms and one interstitial diethyl ether molecule as are omitted for clarity. 


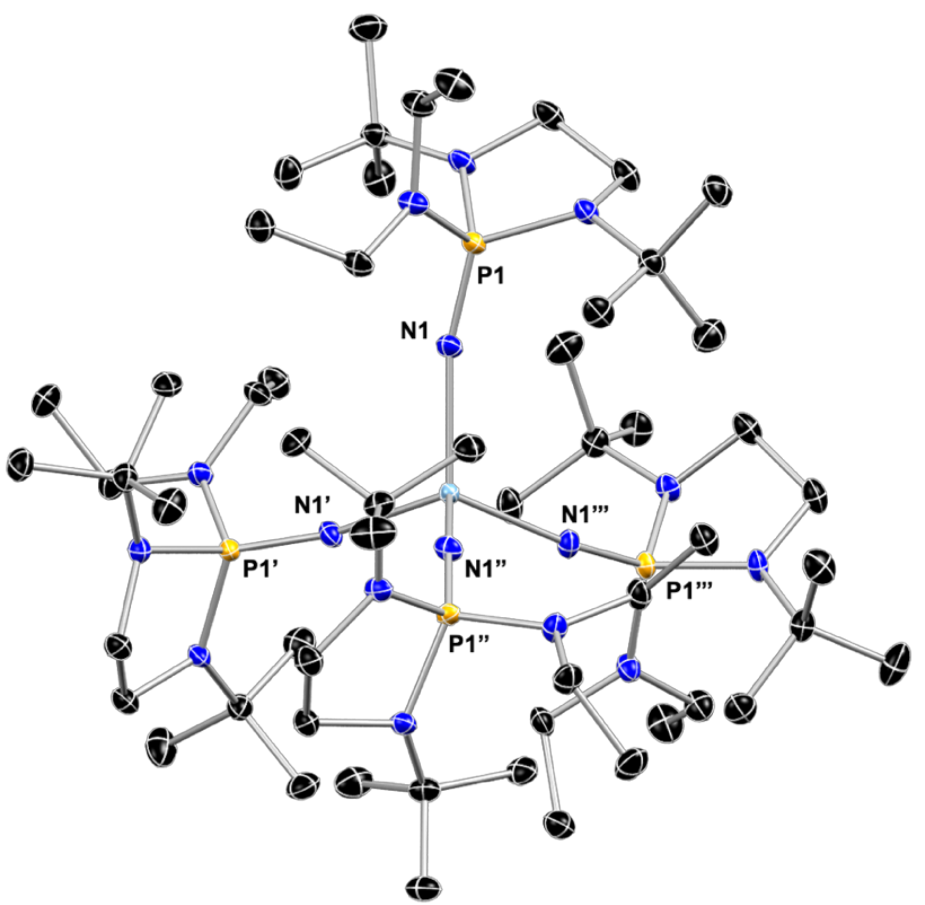

Figure S27. Molecular structure of 4 with thermal ellipsoids shown at 50\% probability. $\mathrm{H}$ atoms are omitted for clarity. 
Table S2. Crystallographic Data

\begin{tabular}{|c|c|c|c|c|c|}
\hline & 2 & 1 & 3 & $3-100 K$ & 4 \\
\hline Empirical Formula & $\mathrm{C}_{116} \mathrm{H}_{266} \mathrm{~K}_{8} \mathrm{~N}_{32} \mathrm{OP}_{8}$ & $\mathrm{C}_{65} \mathrm{H}_{129} \mathrm{KN}_{15} \mathrm{OP}_{4} \mathrm{~Tb}$ & $\mathrm{C}_{60} \mathrm{H}_{138} \mathrm{KN}_{16} \mathrm{OP}_{4} \mathrm{~Tb}$ & $\mathrm{C}_{64} \mathrm{H}_{148} \mathrm{~N}_{16} \mathrm{O}_{2} \mathrm{P}_{4} \mathrm{~Tb}$ & $\mathrm{C}_{66} \mathrm{H}_{152} \mathrm{~N}_{16} \mathrm{P} 4 \mathrm{~Tb}$ \\
\hline Formula Weight & 2686.15 & 1458.72 & 1421.76 & 1495.88 & 1452.83 \\
\hline Temperature (K) & $100(2)$ & $100(2)$ & $180(2)$ & $100(2)$ & $107(2)$ \\
\hline Crystal System & monoclinic & monoclinic & monoclinic & monoclinic & tetragonal \\
\hline Space Group & $\mathrm{P} 2{ }_{1} / c$ & $C c$ & $\mathrm{C} 2 / c$ & $\mathrm{P} 2_{1} / \mathrm{n}$ & I-4 \\
\hline $\mathbf{a} / \mathbf{A}$ & $22.6075(3)$ & $24.279(5)$ & $22.4833(11)$ & $22.587(3)$ & $13.2756(8)$ \\
\hline $\mathbf{b} / \AA$ & $14.13495(15)$ & $14.385(3)$ & $15.9381(8)$ & $15.454(2)$ & $13.2756(8)$ \\
\hline $\mathbf{c} / \AA \AA$ & $24.5014(3)$ & $23.002(5)$ & $25.5112(16)$ & $25.702(4)$ & 21.077(3) \\
\hline$\alpha /{ }^{\circ}$ & 90 & 90 & 90 & 90 & 90 \\
\hline$\beta / \circ$ & $105.3668(12)$ & $108.768(7)$ & $114.512(2)$ & $114.727(5)$ & 90 \\
\hline$\gamma / \circ$ & 90 & 90 & 90 & 90 & 90 \\
\hline Volume $/ \AA^{3}$ & $7549.65(16)$ & $7606(3)$ & $8317.8(8)$ & $3714.6(7)$ & $3714.6(7)$ \\
\hline $\mathbf{Z}$ & 2 & 4 & 4 & 4 & 2 \\
\hline $\mathbf{Z}^{\prime}$ & 0.5 & 1 & 0.5 & 1 & 0.25 \\
\hline$\rho\left(\mathrm{g} / \mathrm{cm}^{3}\right)$ & 1.182 & 1.276 & 1.135 & 1.219 & 1.299 \\
\hline$\mu\left(\mathrm{mm}^{-1}\right)$ & 0.367 & 1.118 & 1.020 & 1.045 & 1.088 \\
\hline$F(000)$ & 2932 & 3104 & 3048 & 3216 & 1570 \\
\hline Crystal Size/mm ${ }^{3}$ & $0.47 \times 0.43 \times 0.33$ & $0.47 \times 0.39 \times 0.20$ & $0.41 \times 0.33 \times 0.32$ & $0.38 \times 0.37 \times 0.28$ & $0.29 \times 0.18 \times 0.13$ \\
\hline Radiation & $\operatorname{MoK} \alpha(\lambda=0.71073)$ & $\operatorname{MoK} \alpha(\lambda=0.71073)$ & $\operatorname{MoK} \alpha(\lambda=0.71073)$ & $\operatorname{MoK} \alpha(\lambda=0.71073)$ & $\operatorname{MoK} \alpha(\lambda=0.71073)$ \\
\hline $\begin{array}{l}2 \theta \text { range for data collection }\left({ }^{\circ}\right) \\
\text { Index Ranges }\end{array}$ & $\begin{array}{c}1.868 \text { to } 36.318 \\
*-36 \leq \mathrm{h} \leq 37,-23 \leq \mathrm{k} \leq 23,-40 \leq 1 \\
\leq 40\end{array}$ & $\begin{array}{c}2.285 \text { to } 31.640 \\
*-35 \leq \mathrm{h} \leq 24,-21 \leq \mathrm{k} \leq \\
21,-33 \leq 1 \leq 33\end{array}$ & $\begin{array}{c}2.702 \text { to } 36.334 \\
*-34 \leq \mathrm{h} \leq 37,-26 \leq \mathrm{k} \leq 26,-40 \leq 1 \leq 42\end{array}$ & $\begin{array}{c}2.72 \text { to } 30.595 \\
*-32 \leq \mathrm{h} \leq 32,-22 \leq \\
\mathrm{k} \leq 21,-36 \leq 1 \leq 36\end{array}$ & $\begin{array}{c}2.906 \text { to } 35.502 \\
*-21 \leq \mathrm{h} \leq 20,-21 \leq \mathrm{k} \leq 16, \\
-33 \leq 1 \leq 34\end{array}$ \\
\hline Reflections Collected & 207068 & 96071 & 79238 & $\begin{array}{c}24862 \\
24826\left[\mathrm{R}_{\text {int }}=\right.\end{array}$ & 27869 \\
\hline Independent Reflections & $\begin{array}{c}36590\left[\mathrm{R}_{\text {int }}=0.0812\right. \\
\left.\mathrm{R}_{\text {sigma }}=0.0508\right]\end{array}$ & $\begin{array}{c}20674\left[\mathrm{R}_{\text {int }}=0.0905\right. \\
\left.\mathrm{R}_{\text {sigma }}=0.093\right]\end{array}$ & $20139\left[\mathrm{R}_{\mathrm{int}}=0.0572, \mathrm{R}_{\text {sigma }}=0.0449\right]$ & $\begin{array}{l}0.0539, \mathrm{R}_{\text {sigma }} \\
\quad=0.0406]\end{array}$ & $\begin{aligned} 8221\left[\mathrm{R}_{\text {int }}\right. & =0.0682, \mathrm{R}_{\text {sigma }} \\
& =0.0713]\end{aligned}$ \\
\hline Data/Restraints/Parameters & 207068/417/797 & $96071 / 607 / 788$ & $79238 / 370 / 410$ & $24826 / 152 / 855$ & $27869 / 34 / 208$ \\
\hline Goodness-of-Fit on $F^{2}$ & 1.048 & $\begin{array}{l}1.017 \\
\mathrm{R}_{l}=0.0576\end{array}$ & 1.042 & $\begin{array}{c}1.219 \\
\mathrm{R}_{l}=00846\end{array}$ & 1.052 \\
\hline Final R Indexes $[\mathrm{I}>=2 \sigma(\mathrm{I})]$ & $\mathrm{R}_{l}=0.0362, w \mathrm{R}_{2}=0.721$ & $\begin{array}{r}w \mathrm{R}_{2}=0.1112 \\
\mathrm{R}_{1}=0.0977, w \mathrm{R}_{2}=\end{array}$ & $\mathrm{R}_{l}=0.0 .0392, \quad w \mathrm{R}_{2}=0.0996$ & $\begin{array}{c}w \mathrm{R}_{2}=0.1845 \\
\mathrm{R}_{1}=0.1015, w \mathrm{R}_{2}=\end{array}$ & $\mathrm{R}_{l}=0.0327, w \mathrm{R}_{2}=0.0863$ \\
\hline Final R Indexes [all data] & $\mathrm{R}_{l}=0.0550, w \mathrm{R}_{2}=0.0392$ & 0.1303 & $\mathrm{R}_{l}=0.0381, w \mathrm{R}_{2}=0.0889$ & 0.1944 & $\mathrm{R}_{l}=0.0375, w \mathrm{R}_{2}=0.0730$ \\
\hline Largest Diff. Peak/Hole/ (e $\left.\AA^{3}\right)$ & $0.699 /-0.599$ & $1.054 /-1.571$ & $1.260 /-1.283$ & $4.30 /-3.65$ & $0.954 /-0.784$ \\
\hline Flack Parameter & - & $0.018(11)$ & - & - & - \\
\hline Completeness to $2 \theta$ & 99.8 & 99.0 & 99.8 & 99.1 & 99.7 \\
\hline
\end{tabular}


Table S3: Bond Lengths in Å for 2.

\begin{tabular}{|c|c|c|}
\hline Atom & Atom & Length/Å \\
\hline$\overline{\mathrm{C} 1}$ & $\mathrm{C} 2$ & $1.5093(13)$ \\
\hline $\mathrm{C} 1$ & $\mathrm{~N} 2$ & $1.4615(12)$ \\
\hline $\mathrm{C} 2$ & N3 & $1.4512(12)$ \\
\hline $\mathrm{C} 3$ & $\mathrm{C} 4$ & $1.5365(14)$ \\
\hline $\mathrm{C} 3$ & $\mathrm{C} 5$ & $1.5379(15)$ \\
\hline $\mathrm{C} 3$ & C6 & $1.5282(14)$ \\
\hline $\mathrm{C} 3$ & $\mathrm{~N} 2$ & $1.4728(12)$ \\
\hline $\mathrm{C} 5$ & K4 & $3.4170(11)$ \\
\hline $\mathrm{C} 7$ & $\mathrm{C} 8$ & $1.5308(15)$ \\
\hline $\mathrm{C} 7$ & $\mathrm{C} 9$ & $1.5322(14)$ \\
\hline $\mathrm{C} 7$ & $\mathrm{C} 10$ & $1.5245(15)$ \\
\hline $\mathrm{C} 7$ & N3 & $1.4709(12)$ \\
\hline C11 & $\mathrm{C} 12$ & $1.5236(14)$ \\
\hline C11 & N4 & $1.4625(11)$ \\
\hline C13 & $\mathrm{C} 14$ & $1.5264(16)$ \\
\hline C13 & N4 & $1.4598(12)$ \\
\hline C13 & K1 & $3.4987(10)$ \\
\hline C14 & K1 & $3.3593(12)$ \\
\hline N1 & P1 & $1.5224(7)$ \\
\hline N1 & K1 & $2.7168(8)$ \\
\hline N1 & $\mathrm{K} 2$ & $2.7308(8)$ \\
\hline N1 & K4 & $2.7263(8)$ \\
\hline N2 & $\mathrm{P} 1$ & $1.7355(8)$ \\
\hline N3 & $\mathrm{P} 1$ & $1.7056(8)$ \\
\hline N4 & $\mathrm{P} 1$ & $1.7127(8)$ \\
\hline P1 & $\mathrm{K} 2$ & $3.7988(3)$ \\
\hline C1D & C2D & $1.5143(14)$ \\
\hline C1D & N2D & $1.4498(12)$ \\
\hline C2D & N3D & $1.4597(12)$ \\
\hline C3D & C4D & $1.5365(14)$ \\
\hline C3D & C5D & $1.5389(14)$ \\
\hline C3D & C6D & $1.5314(15)$ \\
\hline C3D & N2D & $1.4686(12)$ \\
\hline C7D & C8D & $1.5315(14)$ \\
\hline C7D & C9D & $1.5379(13)$ \\
\hline C7D & C10D & $1.5301(14)$ \\
\hline C7D & N3D & $1.4798(12)$ \\
\hline C11D & C12D & $1.5224(14)$ \\
\hline C11D & N4D & $1.4584(11)$ \\
\hline C13D & C14D & $1.5278(14)$ \\
\hline C13D & N4D & $1.4536(12)$ \\
\hline C14D & $\mathrm{K} 1$ & $3.4174(11)$ \\
\hline N1D & P1D & $1.5221(7)$ \\
\hline N1D & K1 & $2.7112(8)$ \\
\hline N1D & $\mathrm{K} 3$ & $2.7299(8)$ \\
\hline N1D & K4 & $2.7100(8)$ \\
\hline N2D & P1D & $1.7132(8)$ \\
\hline N3D & P1D & $1.7177(8)$ \\
\hline N4D & P1D & $1.7001(8)$ \\
\hline P1D & K3 & $3.8658(3)$ \\
\hline P1D & K4 & $3.8110(3)$ \\
\hline $\mathrm{C} 1 \mathrm{C}$ & $\mathrm{C} 2 \mathrm{C}$ & $1.5102(15)$ \\
\hline $\mathrm{C} 1 \mathrm{C}$ & $\mathrm{N} 2 \mathrm{C}$ & $1.4546(12)$ \\
\hline $\mathrm{C} 2 \mathrm{C}$ & $\mathrm{N} 3 \mathrm{C}$ & $1.4583(12)$ \\
\hline $\mathrm{C} 3 \mathrm{C}$ & $\mathrm{C} 4 \mathrm{C}$ & $1.5340(15)$ \\
\hline $\mathrm{C} 3 \mathrm{C}$ & $\mathrm{C} 5 \mathrm{C}$ & $1.5368(15)$ \\
\hline $\mathrm{C} 3 \mathrm{C}$ & $\mathrm{C} 6 \mathrm{C}$ & $1.5311(15)$ \\
\hline $\mathrm{C} 3 \mathrm{C}$ & $\mathrm{N} 2 \mathrm{C}$ & $1.4805(12)$ \\
\hline
\end{tabular}

\begin{tabular}{|c|c|c|}
\hline Atom & Atom & Length/Å \\
\hline$\overline{\mathrm{C} 5 \mathrm{C}}$ & K2 & $3.4106(12)$ \\
\hline $\mathrm{C} 6 \mathrm{C}$ & $\mathrm{K} 2$ & $3.5283(11)$ \\
\hline $\mathrm{C} 7 \mathrm{C}$ & $\mathrm{C} 8 \mathrm{C}$ & $1.5325(15)$ \\
\hline $\mathrm{C} 7 \mathrm{C}$ & $\mathrm{C} 9 \mathrm{C}$ & $1.5377(14)$ \\
\hline $\mathrm{C} 7 \mathrm{C}$ & $\mathrm{C} 10 \mathrm{C}$ & $1.5316(15)$ \\
\hline $\mathrm{C} 7 \mathrm{C}$ & N3C & $1.4833(12)$ \\
\hline $\mathrm{C} 11 \mathrm{C}$ & $\mathrm{C} 12 \mathrm{C}$ & $1.5223(15)$ \\
\hline $\mathrm{C} 11 \mathrm{C}$ & N4C & $1.4566(12)$ \\
\hline $\mathrm{C} 13 \mathrm{C}$ & $\mathrm{C} 14 \mathrm{C}$ & $1.5222(15)$ \\
\hline $\mathrm{C} 13 \mathrm{C}$ & N4C & $1.4519(12)$ \\
\hline N1C & $\mathrm{P} 1 \mathrm{C}$ & $1.5223(8)$ \\
\hline $\mathrm{N} 1 \mathrm{C}$ & $\mathrm{K} 2$ & $2.7313(7)$ \\
\hline $\mathrm{N} 1 \mathrm{C}$ & $\mathrm{K} 3$ & $2.7056(8)$ \\
\hline $\mathrm{N} 1 \mathrm{C}$ & K4 & $2.6826(8)$ \\
\hline $\mathrm{N} 2 \mathrm{C}$ & $\mathrm{P} 1 \mathrm{C}$ & $1.7244(8)$ \\
\hline $\mathrm{N} 3 \mathrm{C}$ & $\mathrm{P} 1 \mathrm{C}$ & $1.7319(8)$ \\
\hline $\mathrm{N} 3 \mathrm{C}$ & K4 & $3.4258(8)$ \\
\hline $\mathrm{N} 4 \mathrm{C}$ & $\mathrm{P} 1 \mathrm{C}$ & $1.7009(8)$ \\
\hline $\mathrm{P} 1 \mathrm{C}$ & K4 & $3.4789(3)$ \\
\hline C1B & $\mathrm{C} 2 \mathrm{~B}$ & $1.5101(16)$ \\
\hline C1B & $\mathrm{N} 2 \mathrm{~B}$ & $1.4562(13)$ \\
\hline $\mathrm{C} 2 \mathrm{~B}$ & N3B & $1.4596(12)$ \\
\hline C3B & C4B & $1.5362(14)$ \\
\hline C3B & C5B & $1.5336(13)$ \\
\hline $\mathrm{C} 3 \mathrm{~B}$ & C6B & $1.5304(14)$ \\
\hline C3B & $\mathrm{N} 2 \mathrm{~B}$ & $1.4778(13)$ \\
\hline $\mathrm{C} 7 \mathrm{~B}$ & C8B & $1.5322(15)$ \\
\hline C7B & C9B & $1.5334(15)$ \\
\hline $\mathrm{C} 7 \mathrm{~B}$ & $\mathrm{C} 10 \mathrm{~B}$ & $1.5295(15)$ \\
\hline C7B & N3B & $1.4812(13)$ \\
\hline C11B & $\mathrm{C} 12 \mathrm{~B}$ & $1.5206(14)$ \\
\hline C11B & N4B & $1.4595(11)$ \\
\hline C13B & C14B & $1.5206(14)$ \\
\hline C13B & N4B & $1.4526(11)$ \\
\hline N1B & P1B & $1.5234(8)$ \\
\hline N1B & K1 & $2.7009(8)$ \\
\hline N1B & K2 & $2.7126(8)$ \\
\hline N1B & $\mathrm{K} 3$ & $2.7096(8)$ \\
\hline $\mathrm{N} 2 \mathrm{~B}$ & P1B & $1.7151(8)$ \\
\hline N3B & P1B & $1.7344(8)$ \\
\hline N4B & P1B & $1.6969(8)$ \\
\hline P1B & K3 & $3.6065(3)$ \\
\hline C1S & $\mathrm{C} 2 \mathrm{~S}$ & $1.519(2)$ \\
\hline C1S & O1S & $1.352(2)$ \\
\hline C3S & $\mathrm{C} 4 \mathrm{~S}$ & $1.516(2)$ \\
\hline $\mathrm{C} 3 \mathrm{~S}$ & O1S & $1.443(2)$ \\
\hline
\end{tabular}


Table S4: Bond Angles in ${ }^{\circ}$ for 2.

\begin{tabular}{|c|c|c|c|}
\hline Atom & Atom & Atom & Angle $/^{\circ}$ \\
\hline N2 & $\mathrm{C} 1$ & $\mathrm{C} 2$ & $104.02(7)$ \\
\hline N3 & $\mathrm{C} 2$ & $\mathrm{C} 1$ & $104.96(7)$ \\
\hline C4 & $\mathrm{C} 3$ & $\mathrm{C} 5$ & $109.41(9)$ \\
\hline C6 & $\mathrm{C} 3$ & $\mathrm{C} 4$ & $107.58(9)$ \\
\hline C6 & $\mathrm{C} 3$ & $\mathrm{C} 5$ & $110.04(8)$ \\
\hline N2 & $\mathrm{C} 3$ & $\mathrm{C} 4$ & $108.89(8)$ \\
\hline N2 & $\mathrm{C} 3$ & $\mathrm{C} 5$ & $111.03(8)$ \\
\hline N2 & $\mathrm{C} 3$ & C6 & $109.82(8)$ \\
\hline C3 & $\mathrm{C} 5$ & K4 & $104.69(6)$ \\
\hline C8 & C7 & C9 & $108.75(9)$ \\
\hline $\mathrm{C} 10$ & $\mathrm{C} 7$ & $\mathrm{C} 8$ & $108.80(9)$ \\
\hline $\mathrm{C} 10$ & C7 & C9 & $107.95(9)$ \\
\hline N3 & C7 & $\mathrm{C} 8$ & $109.35(9)$ \\
\hline N3 & C7 & C9 & $110.60(8)$ \\
\hline N3 & $\mathrm{C} 7$ & $\mathrm{C} 10$ & $111.34(8)$ \\
\hline N4 & C11 & $\mathrm{C} 12$ & $114.47(8)$ \\
\hline C14 & C13 & K1 & $72.08(6)$ \\
\hline N4 & C13 & $\mathrm{C} 14$ & $113.57(9)$ \\
\hline N4 & C13 & K1 & $103.90(5)$ \\
\hline $\mathrm{C} 13$ & C14 & K1 & $82.30(6)$ \\
\hline P1 & N1 & K1 & $131.40(4)$ \\
\hline P1 & N1 & $\mathrm{K} 2$ & $124.05(4)$ \\
\hline $\mathrm{P} 1$ & N1 & K4 & $135.82(4)$ \\
\hline K1 & N1 & K2 & $81.08(2)$ \\
\hline K1 & N1 & K4 & $82.81(2)$ \\
\hline K4 & N1 & K2 & $82.41(2)$ \\
\hline $\mathrm{C} 1$ & N2 & C3 & $115.96(7)$ \\
\hline $\mathrm{C} 1$ & N2 & P1 & $109.54(6)$ \\
\hline C3 & N2 & P1 & $121.44(6)$ \\
\hline $\mathrm{C} 2$ & N3 & $\mathrm{C} 7$ & $117.39(7)$ \\
\hline C2 & N3 & P1 & $114.18(6)$ \\
\hline C7 & N3 & P1 & $127.46(7)$ \\
\hline C11 & N4 & P1 & $119.46(6)$ \\
\hline $\mathrm{C} 13$ & N4 & $\mathrm{C} 11$ & $115.06(7)$ \\
\hline $\mathrm{C} 13$ & N4 & P1 & 119.11(6) \\
\hline N1 & $\mathrm{P} 1$ & $\mathrm{~N} 2$ & $122.78(4)$ \\
\hline N1 & P1 & N3 & $120.11(4)$ \\
\hline N1 & P1 & N4 & $113.65(4)$ \\
\hline N1 & P1 & $\mathrm{K} 2$ & $36.55(3)$ \\
\hline N2 & P1 & $\mathrm{K} 2$ & $122.66(3)$ \\
\hline N3 & P1 & N2 & $90.17(4)$ \\
\hline N3 & P1 & N4 & $104.16(4)$ \\
\hline N3 & P1 & $\mathrm{K} 2$ & $84.02(3)$ \\
\hline N4 & P1 & N2 & $102.05(4)$ \\
\hline N4 & P1 & $\mathrm{K} 2$ & $134.72(3)$ \\
\hline N2D & C1D & C2D & $105.41(7)$ \\
\hline N3D & C2D & C1D & $104.85(8)$ \\
\hline C4D & $\mathrm{C} 3 \mathrm{D}$ & C5D & $108.60(8)$ \\
\hline C6D & C3D & C4D & 107.61(9) \\
\hline C6D & C3D & C5D & $109.50(8)$ \\
\hline N2D & C3D & C4D & $110.22(8)$ \\
\hline N2D & C3D & C5D & $109.97(8)$ \\
\hline N2D & C3D & C6D & $110.89(8)$ \\
\hline C8D & C7D & C9D & $109.04(8)$ \\
\hline C10D & C7D & C8D & $108.27(8)$ \\
\hline C10D & C7D & C9D & $107.79(9)$ \\
\hline N3D & C7D & C8D & $111.75(8)$ \\
\hline N3D & C7D & C9D & $108.48(8)$ \\
\hline
\end{tabular}

\begin{tabular}{|c|c|c|c|}
\hline Atom & Atom & Atom & Angle $/^{\circ}$ \\
\hline N3D & C7D & C10D & $111.41(7)$ \\
\hline N4D & C11D & C12D & $113.79(8)$ \\
\hline N4D & C13D & C14D & $114.66(8)$ \\
\hline C13D & C14D & K1 & $82.09(5)$ \\
\hline P1D & N1D & K1 & $136.53(4)$ \\
\hline P1D & N1D & K3 & $128.51(4)$ \\
\hline P1D & N1D & K4 & $126.12(4)$ \\
\hline K1 & N1D & K3 & $81.78(2)$ \\
\hline K4 & N1D & K1 & $83.22(2)$ \\
\hline K4 & N1D & $\mathrm{K} 3$ & $81.99(2)$ \\
\hline C1D & $\mathrm{N} 2 \mathrm{D}$ & C3D & $117.57(7)$ \\
\hline C1D & N2D & P1D & $114.62(6)$ \\
\hline C3D & N2D & P1D & $127.81(6)$ \\
\hline C2D & N3D & C7D & $116.34(7)$ \\
\hline C2D & N3D & P1D & $109.73(6)$ \\
\hline C7D & N3D & P1D & $126.01(6)$ \\
\hline C11D & N4D & P1D & $120.32(6)$ \\
\hline C13D & N4D & C11D & $115.50(8)$ \\
\hline C13D & N4D & P1D & $119.25(6)$ \\
\hline N1D & P1D & $\mathrm{N} 2 \mathrm{D}$ & $119.82(4)$ \\
\hline N1D & P1D & N3D & $123.66(4)$ \\
\hline N1D & P1D & N4D & $113.73(4)$ \\
\hline N1D & P1D & K3 & $33.54(3)$ \\
\hline N1D & P1D & K4 & $35.06(3)$ \\
\hline N2D & P1D & N3D & $90.01(4)$ \\
\hline N2D & P1D & K3 & $87.13(3)$ \\
\hline N2D & P1D & K4 & $117.79(3)$ \\
\hline N3D & P1D & $\mathrm{K} 3$ & $137.49(3)$ \\
\hline N3D & P1D & K4 & $89.61(3)$ \\
\hline N4D & P1D & $\mathrm{N} 2 \mathrm{D}$ & $105.60(4)$ \\
\hline N4D & P1D & N3D & $99.99(4)$ \\
\hline N4D & P1D & K3 & $121.60(3)$ \\
\hline N4D & P1D & $\mathrm{K} 4$ & $135.55(3)$ \\
\hline K4 & P1D & K3 & $55.396(5)$ \\
\hline $\mathrm{N} 2 \mathrm{C}$ & $\mathrm{C} 1 \mathrm{C}$ & $\mathrm{C} 2 \mathrm{C}$ & $105.32(8)$ \\
\hline N3C & $\mathrm{C} 2 \mathrm{C}$ & $\mathrm{C} 1 \mathrm{C}$ & $104.92(8)$ \\
\hline $\mathrm{C} 4 \mathrm{C}$ & $\mathrm{C} 3 \mathrm{C}$ & $\mathrm{C} 5 \mathrm{C}$ & 108.91(9) \\
\hline $\mathrm{C} 6 \mathrm{C}$ & $\mathrm{C} 3 \mathrm{C}$ & $\mathrm{C} 4 \mathrm{C}$ & $107.75(9)$ \\
\hline C6C & $\mathrm{C} 3 \mathrm{C}$ & $\mathrm{C} 5 \mathrm{C}$ & $109.62(9)$ \\
\hline $\mathrm{N} 2 \mathrm{C}$ & $\mathrm{C} 3 \mathrm{C}$ & $\mathrm{C} 4 \mathrm{C}$ & 108.71(9) \\
\hline $\mathrm{N} 2 \mathrm{C}$ & $\mathrm{C} 3 \mathrm{C}$ & $\mathrm{C} 5 \mathrm{C}$ & $111.34(8)$ \\
\hline $\mathrm{N} 2 \mathrm{C}$ & $\mathrm{C} 3 \mathrm{C}$ & $\mathrm{C} 6 \mathrm{C}$ & $110.42(8)$ \\
\hline $\mathrm{C} 3 \mathrm{C}$ & $\mathrm{C} 5 \mathrm{C}$ & $\mathrm{K} 2$ & $99.08(6)$ \\
\hline $\mathrm{C} 3 \mathrm{C}$ & C6C & K2 & $94.54(6)$ \\
\hline $\mathrm{C} 8 \mathrm{C}$ & C7C & C9C & $108.52(9)$ \\
\hline $\mathrm{C} 10 \mathrm{C}$ & C7C & $\mathrm{C} 8 \mathrm{C}$ & $108.00(9)$ \\
\hline $\mathrm{C} 10 \mathrm{C}$ & $\mathrm{C} 7 \mathrm{C}$ & C9C & 108.11(9) \\
\hline N3C & $\mathrm{C} 7 \mathrm{C}$ & $\mathrm{C} 8 \mathrm{C}$ & $108.80(9)$ \\
\hline $\mathrm{N} 3 \mathrm{C}$ & C7C & C9C & $112.48(8)$ \\
\hline $\mathrm{N} 3 \mathrm{C}$ & C7C & $\mathrm{C} 10 \mathrm{C}$ & $110.81(8)$ \\
\hline $\mathrm{N} 4 \mathrm{C}$ & $\mathrm{C} 11 \mathrm{C}$ & $\mathrm{C} 12 \mathrm{C}$ & $114.78(8)$ \\
\hline $\mathrm{N} 4 \mathrm{C}$ & $\mathrm{C} 13 \mathrm{C}$ & $\mathrm{C} 14 \mathrm{C}$ & $114.07(8)$ \\
\hline $\mathrm{P} 1 \mathrm{C}$ & $\mathrm{N} 1 \mathrm{C}$ & K2 & $133.98(4)$ \\
\hline $\mathrm{P} 1 \mathrm{C}$ & $\mathrm{N} 1 \mathrm{C}$ & K3 & $141.14(4)$ \\
\hline $\mathrm{P} 1 \mathrm{C}$ & $\mathrm{N} 1 \mathrm{C}$ & K4 & $108.48(4)$ \\
\hline K3 & $\mathrm{N} 1 \mathrm{C}$ & K2 & $83.21(2)$ \\
\hline K4 & $\mathrm{N} 1 \mathrm{C}$ & $\mathrm{K} 2$ & $83.21(2)$ \\
\hline K4 & $\mathrm{N} 1 \mathrm{C}$ & $\mathrm{K} 3$ & $82.95(2)$ \\
\hline $\mathrm{C} 1 \mathrm{C}$ & $\mathrm{N} 2 \mathrm{C}$ & $\mathrm{C} 3 \mathrm{C}$ & $115.40(8)$ \\
\hline
\end{tabular}




\begin{tabular}{|c|c|c|c|}
\hline Atom & Atom & Atom & Angle $/^{\circ}$ \\
\hline$\overline{\mathrm{C} 1 \mathrm{C}}$ & $\mathrm{N} 2 \mathrm{C}$ & $\mathrm{P} 1 \mathrm{C}$ & $111.32(6)$ \\
\hline $\mathrm{C} 3 \mathrm{C}$ & $\mathrm{N} 2 \mathrm{C}$ & $\mathrm{P} 1 \mathrm{C}$ & $121.62(6)$ \\
\hline $\mathrm{C} 2 \mathrm{C}$ & $\mathrm{N} 3 \mathrm{C}$ & C7C & $115.86(8)$ \\
\hline $\mathrm{C} 2 \mathrm{C}$ & $\mathrm{N} 3 \mathrm{C}$ & $\mathrm{P} 1 \mathrm{C}$ & $112.34(6)$ \\
\hline $\mathrm{C} 2 \mathrm{C}$ & $\mathrm{N} 3 \mathrm{C}$ & K4 & $121.88(6)$ \\
\hline C7C & $\mathrm{N} 3 \mathrm{C}$ & $\mathrm{P} 1 \mathrm{C}$ & $124.88(6)$ \\
\hline $\mathrm{C} 7 \mathrm{C}$ & $\mathrm{N} 3 \mathrm{C}$ & K4 & $98.13(5)$ \\
\hline $\mathrm{P} 1 \mathrm{C}$ & $\mathrm{N} 3 \mathrm{C}$ & K4 & $77.18(3)$ \\
\hline $\mathrm{C} 11 \mathrm{C}$ & $\mathrm{N} 4 \mathrm{C}$ & $\mathrm{P} 1 \mathrm{C}$ & $123.83(6)$ \\
\hline $\mathrm{C} 13 \mathrm{C}$ & $\mathrm{N} 4 \mathrm{C}$ & $\mathrm{C} 11 \mathrm{C}$ & $116.14(7)$ \\
\hline $\mathrm{C} 13 \mathrm{C}$ & $\mathrm{N} 4 \mathrm{C}$ & P1C & 119.71(6) \\
\hline N1C & $\mathrm{P} 1 \mathrm{C}$ & $\mathrm{N} 2 \mathrm{C}$ & $122.40(4)$ \\
\hline $\mathrm{N} 1 \mathrm{C}$ & $\mathrm{P} 1 \mathrm{C}$ & $\mathrm{N} 3 \mathrm{C}$ & $117.73(4)$ \\
\hline $\mathrm{N} 1 \mathrm{C}$ & $\mathrm{P} 1 \mathrm{C}$ & $\mathrm{N} 4 \mathrm{C}$ & $114.00(4)$ \\
\hline $\mathrm{N} 1 \mathrm{C}$ & $\mathrm{P} 1 \mathrm{C}$ & K4 & $47.00(3)$ \\
\hline $\mathrm{N} 2 \mathrm{C}$ & $\mathrm{P} 1 \mathrm{C}$ & $\mathrm{N} 3 \mathrm{C}$ & $90.98(4)$ \\
\hline $\mathrm{N} 2 \mathrm{C}$ & $\mathrm{P} 1 \mathrm{C}$ & K4 & $110.14(3)$ \\
\hline $\mathrm{N} 3 \mathrm{C}$ & $\mathrm{P} 1 \mathrm{C}$ & K4 & $73.78(3)$ \\
\hline $\mathrm{N} 4 \mathrm{C}$ & $\mathrm{P} 1 \mathrm{C}$ & $\mathrm{N} 2 \mathrm{C}$ & $101.24(4)$ \\
\hline $\mathrm{N} 4 \mathrm{C}$ & $\mathrm{P} 1 \mathrm{C}$ & $\mathrm{N} 3 \mathrm{C}$ & $107.14(4)$ \\
\hline $\mathrm{N} 4 \mathrm{C}$ & $\mathrm{P} 1 \mathrm{C}$ & K4 & $148.61(3)$ \\
\hline N2B & $\mathrm{C} 1 \mathrm{~B}$ & $\mathrm{C} 2 \mathrm{~B}$ & $104.76(8)$ \\
\hline N3B & $\mathrm{C} 2 \mathrm{~B}$ & $\mathrm{C} 1 \mathrm{~B}$ & $104.44(8)$ \\
\hline $\mathrm{C} 5 \mathrm{~B}$ & $\mathrm{C} 3 \mathrm{~B}$ & C4B & $108.53(8)$ \\
\hline C6B & C3B & C4B & $108.75(9)$ \\
\hline C6B & C3B & C5B & $107.67(8)$ \\
\hline $\mathrm{N} 2 \mathrm{~B}$ & C $3 B$ & $\mathrm{C} 4 \mathrm{~B}$ & $111.54(8)$ \\
\hline N2B & C3B & C5B & $109.02(8)$ \\
\hline N2B & C3B & C6B & $111.23(7)$ \\
\hline C8B & C7B & C9B & $109.02(9)$ \\
\hline C10B & C7B & $\mathrm{C} 8 \mathrm{~B}$ & $109.09(9)$ \\
\hline $\mathrm{C} 10 \mathrm{~B}$ & C7B & C9B & $108.18(9)$ \\
\hline N3B & C7B & C8B & $111.51(9)$ \\
\hline N3B & C7B & C9B & $109.09(9)$ \\
\hline N3B & C7B & $\mathrm{C} 10 \mathrm{~B}$ & $109.88(8)$ \\
\hline N4B & C11B & $\mathrm{C} 12 \mathrm{~B}$ & $115.47(8)$ \\
\hline N4B & C13B & C14B & $113.96(8)$ \\
\hline P1B & N1B & K1 & $143.68(4)$ \\
\hline P1B & N1B & K2 & $130.36(4)$ \\
\hline P1B & N1B & K3 & $113.90(4)$ \\
\hline K1 & N1B & K2 & $81.70(2)$ \\
\hline K1 & N1B & K3 & $82.35(2)$ \\
\hline K3 & N1B & K2 & $83.48(2)$ \\
\hline $\mathrm{C} 1 \mathrm{~B}$ & $\mathrm{~N} 2 \mathrm{~B}$ & C3B & $117.18(8)$ \\
\hline C1B & $\mathrm{N} 2 \mathrm{~B}$ & P1B & $113.27(7)$ \\
\hline C3B & N2B & P1B & $127.45(6)$ \\
\hline $\mathrm{C} 2 \mathrm{~B}$ & N3B & C7B & $115.71(8)$ \\
\hline $\mathrm{C} 2 \mathrm{~B}$ & N3B & P1B & $109.67(6)$ \\
\hline C7B & N3B & P1B & $121.02(6)$ \\
\hline C11B & N4B & P1B & $122.82(6)$ \\
\hline C13B & N4B & C11B & $115.79(7)$ \\
\hline C13B & N4B & P1B & $120.33(6)$ \\
\hline N1B & P1B & N2B & $119.53(4)$ \\
\hline N1B & P1B & $\mathrm{N} 3 \mathrm{~B}$ & $121.98(4)$ \\
\hline N1B & P1B & N4B & $113.66(4)$ \\
\hline N1B & P1B & K3 & $43.38(3)$ \\
\hline N2B & P1B & N3B & $90.71(4)$ \\
\hline N2B & P1B & K3 & 78.33(3) \\
\hline N3B & P1B & K3 & $113.56(3)$ \\
\hline
\end{tabular}

\begin{tabular}{llll}
\hline Atom & Atom & Atom & \multicolumn{1}{|c}{ Angle ${ }^{\circ}$} \\
\hline N4B & P1B & N2B & $104.73(4)$ \\
N4B & P1B & N3B & $102.61(4)$ \\
N4B & P1B & K3 & $143.72(3)$ \\
O1S & C1S & C2S & $106.7(2)$ \\
O1S & C3S & C4S & $108.29(19)$ \\
C1S & O1S & C3S & $115.33(14)$ \\
N1 & K1 & C14 & $78.32(3)$ \\
N1D & K1 & C14 & $126.04(3)$ \\
N1D & K1 & N1 & $96.28(2)$ \\
N1B & K1 & C14 & $136.13(3)$ \\
N1B & K1 & N1 & $98.62(2)$ \\
N1B & K1 & N1D & $97.83(2)$ \\
N1 & K2 & C5C & $88.91(3)$ \\
N1 & K2 & N1C & $96.12(2)$ \\
N1C & K2 & C5C & $72.78(2)$ \\
N1B & K2 & N1 & $97.99(2)$ \\
N1B & K2 & C5C & $167.28(3)$ \\
N1B & K2 & N1C & $95.76(2)$ \\
N1D & K3 & K1 & $48.882(16)$ \\
N1C & K3 & N1D & $96.69(2)$ \\
N1C & K3 & N1B & $96.44(2)$ \\
N1C & K3 & K1 & $94.613(17)$ \\
N1B & K3 & N1D & $97.17(2)$ \\
N1B & K3 & K1 & $48.723(17)$ \\
N1 & K4 & C5 & $70.85(2)$ \\
N1D & K4 & C5 & $150.86(3)$ \\
N1D & K4 & N1 & $96.08(2)$ \\
N1C & K4 & C5 & $109.50(3)$ \\
N1C & K4 & N1 & $97.38(2)$ \\
N1C & K4 & N1D & $97.72(2)$ \\
& & &
\end{tabular}


Table S5: Bond Lengths in $\AA$ for 1.

\begin{tabular}{|c|c|c|}
\hline Atom & Atom & Length/Å \\
\hline $\mathrm{Tb} 1$ & $\mathrm{~K} 1$ & $3.2285(18)$ \\
\hline Tb1 & N6 & $2.235(8)$ \\
\hline Tb1 & N2 & $2.239(6)$ \\
\hline Tb1 & N1 & $2.241(6)$ \\
\hline Tb1 & $\mathrm{N} 12$ & $2.200(7)$ \\
\hline K1 & $\mathrm{O} 1$ & $2.771(7)$ \\
\hline K1 & N6 & $3.015(7)$ \\
\hline K1 & N2 & $2.780(7)$ \\
\hline K1 & N1 & $2.797(8)$ \\
\hline P3 & N9 & $1.677(7)$ \\
\hline P3 & N10 & $1.697(6)$ \\
\hline P3 & N1 & $1.534(8)$ \\
\hline P3 & N11 & $1.698(7)$ \\
\hline P1 & N3 & $1.701(8)$ \\
\hline P1 & N4 & $1.689(8)$ \\
\hline P1 & N2 & $1.532(7)$ \\
\hline P1 & N5 & $1.693(7)$ \\
\hline P4 & N13 & $1.667(9)$ \\
\hline P4 & N14 & $1.683(7)$ \\
\hline P4 & N15 & $1.703(7)$ \\
\hline P4 & N12 & $1.523(7)$ \\
\hline $\mathrm{P} 2$ & N6 & $1.516(7)$ \\
\hline P2 & N7 & $1.680(10)$ \\
\hline $\mathrm{P} 2$ & N8 & $1.705(8)$ \\
\hline $\mathrm{P} 2$ & $\mathrm{C} 30$ & $1.686(9)$ \\
\hline $\mathrm{O} 1$ & $\mathrm{C} 1$ & $1.444(14)$ \\
\hline O1 & $\mathrm{C} 3$ & $1.417(15)$ \\
\hline N13 & C51 & $1.480(12)$ \\
\hline N13 & C55 & $1.476(11)$ \\
\hline N3 & $\mathrm{C} 5$ & $1.483(10)$ \\
\hline N3 & C9 & $1.460(12)$ \\
\hline N14 & C56 & $1.434(12)$ \\
\hline N14 & $\mathrm{C} 60$ & $1.459(10)$ \\
\hline N7 & $\mathrm{C} 20$ & $1.444(13)$ \\
\hline N7 & $\mathrm{C} 24$ & $1.486(12)$ \\
\hline N9 & $\mathrm{C} 36$ & $1.438(14)$ \\
\hline N9 & $\mathrm{C} 40$ & $1.462(10)$ \\
\hline N10 & $\mathrm{C} 41$ & $1.459(11)$ \\
\hline N10 & $\mathrm{C} 45$ & $1.456(10)$ \\
\hline N4 & $\mathrm{C} 10$ & $1.456(12)$ \\
\hline N4 & $\mathrm{C} 14$ & $1.467(14)$ \\
\hline N15 & C61 & $1.481(10)$ \\
\hline N15 & C65 & $1.455(12)$ \\
\hline C39 & C38 & $1.520(13)$ \\
\hline C39 & $\mathrm{C} 40$ & $1.513(11)$ \\
\hline N8 & $\mathrm{C} 25$ & $1.485(15)$ \\
\hline N8 & $\mathrm{C} 29$ & $1.454(12)$ \\
\hline N11 & $\mathrm{C} 46$ & $1.462(10)$ \\
\hline N11 & $\mathrm{C} 50$ & $1.484(10)$ \\
\hline N5 & $\mathrm{C} 15$ & $1.459(10)$ \\
\hline N5 & C19 & $1.473(12)$ \\
\hline C34 & C33 & $1.517(12)$ \\
\hline C34 & $\mathrm{C} 35$ & $1.469(13)$ \\
\hline $\mathrm{C} 15$ & $\mathrm{C} 16$ & $1.523(11)$ \\
\hline C19 & C18 & $1.502(13)$ \\
\hline C49 & $\mathrm{C} 50$ & $1.508(12)$ \\
\hline C49 & $\mathrm{C} 48$ & $1.522(12)$ \\
\hline
\end{tabular}

\begin{tabular}{lll}
\hline Atom & Atom & Length/A \\
\hline C42 & C43 & $1.513(12)$ \\
C42 & C41 & $1.499(11)$ \\
C46 & C47 & $1.509(12)$ \\
C64 & C65 & $1.532(11)$ \\
C64 & C63 & $1.506(11)$ \\
C43 & C44 & $1.512(13)$ \\
C7 & C8 & $1.534(13)$ \\
C7 & C6 & $1.528(16)$ \\
C61 & C62 & $1.501(11)$ \\
C38 & C37 & $1.521(11)$ \\
C36 & C37 & $1.533(13)$ \\
C31 & C30 & $1.473(11)$ \\
C31 & C32 & $1.468(14)$ \\
C33 & C32 & $1.474(13)$ \\
C56 & C57 & $1.475(14)$ \\
C45 & C44 & $1.497(12)$ \\
C8 & C9 & $1.513(14)$ \\
C60 & C59 & $1.493(14)$ \\
C27 & C26 & $1.514(14)$ \\
C27 & C28 & $1.526(17)$ \\
C47 & C48 & $1.506(12)$ \\
C6 & C5 & $1.513(14)$ \\
C10 & C11 & $1.459(15)$ \\
C25 & C26 & $1.518(12)$ \\
C30 & C35 & $1.503(13)$ \\
C16 & C17 & $1.524(14)$ \\
C20 & C21 & $1.528(15)$ \\
C51 & C52 & $1.492(15)$ \\
C55 & C54 & $1.524(14)$ \\
C59 & C58 & $1.484(15)$ \\
C54 & C53 & $1.532(15)$ \\
C28 & C29 & $1.525(12)$ \\
C14 & C13 & $1.407(17)$ \\
C57 & C58 & $1.500(13)$ \\
C53 & C52 & $1.529(14)$ \\
C13 & C12 & $1.516(14)$ \\
C1 & C2 & $1.468(18)$ \\
C11 & C12 & $1.513(18)$ \\
C4 & C3 & $1.496(16)$ \\
C23 & C24 & $1.440(16)$ \\
C23 & C22 & $1.502(17)$ \\
C63 & C62 & $1.513(13)$ \\
C18 & C17 & $1.516(13)$ \\
C21 & C22 & $1.564(15)$ \\
& &
\end{tabular}

Table S6: Bond Angles in ${ }^{\circ} \mathbf{1 .}$

\begin{tabular}{llll}
\hline Atom & Atom & Atom & \multicolumn{1}{c}{ Angle ${ }^{\circ}$} \\
\hline N6 & Tb1 & K1 & $64.01(17)$ \\
N6 & Tb1 & N2 & $95.8(3)$ \\
N6 & Tb1 & N1 & $98.5(3)$ \\
N2 & Tb1 & K1 & $57.78(17)$ \\
N2 & Tb1 & N1 & $97.1(2)$ \\
N1 & Tb1 & K1 & $58.21(19)$ \\
N12 & Tb1 & K1 & $172.1(2)$ \\
N12 & Tb1 & N6 & $123.3(3)$ \\
N12 & Tb1 & N2 & $121.2(2)$ \\
N12 & Tb1 & N1 & $115.5(3)$
\end{tabular}




\begin{tabular}{|c|c|c|c|c|c|c|c|}
\hline Atom & Atom & Atom & Angle ${ }^{\circ}$ & Atom & Atom & Atom & Angle $~^{\circ}$ \\
\hline $\mathrm{O} 1$ & K1 & N6 & $149.2(2)$ & $\bar{C} 65$ & N15 & C61 & $110.2(7)$ \\
\hline $\mathrm{O} 1$ & K1 & N2 & $111.38(19)$ & Tb1 & N2 & K1 & $79.27(18)$ \\
\hline $\mathrm{O} 1$ & K1 & N1 & $139.5(2)$ & P1 & N2 & Tb1 & $135.2(4)$ \\
\hline N2 & K1 & N6 & $69.76(18)$ & P1 & N2 & K1 & $130.7(4)$ \\
\hline N2 & $\mathrm{K} 1$ & N1 & $74.0(2)$ & $\mathrm{C} 40$ & C39 & C38 & $110.9(7)$ \\
\hline N1 & K1 & N6 & $71.2(2)$ & Tb1 & N1 & $\mathrm{K} 1$ & $78.9(2)$ \\
\hline N9 & P3 & N10 & $108.5(4)$ & P3 & N1 & Tb1 & $135.8(4)$ \\
\hline N9 & P3 & N11 & $100.8(4)$ & P3 & N1 & K1 & $128.0(3)$ \\
\hline N10 & P3 & N11 & $99.7(3)$ & $\mathrm{C} 25$ & N8 & $\mathrm{P} 2$ & 111.7(6) \\
\hline N1 & P3 & N9 & $113.8(4)$ & $\mathrm{C} 29$ & N8 & P2 & $115.9(6)$ \\
\hline N1 & P3 & N10 & $112.5(4)$ & C29 & N8 & $\mathrm{C} 25$ & $109.2(8)$ \\
\hline N1 & P3 & N11 & $120.0(3)$ & P4 & N12 & Tb1 & $172.5(5)$ \\
\hline N4 & P1 & N3 & $100.7(4)$ & C46 & N11 & P3 & $114.2(5)$ \\
\hline N4 & P1 & N5 & 108.1(4) & C46 & N11 & C50 & $109.6(6)$ \\
\hline N2 & P1 & N3 & 121.1(3) & C50 & N11 & P3 & $114.4(6)$ \\
\hline N2 & P1 & N4 & $113.0(4)$ & $\mathrm{C} 15$ & N5 & P1 & 121.1(5) \\
\hline N2 & P1 & N5 & $112.1(3)$ & $\mathrm{C} 15$ & N5 & C19 & $110.8(6)$ \\
\hline N5 & P1 & N3 & $100.4(4)$ & C19 & N5 & P1 & $114.3(6)$ \\
\hline N13 & P4 & N14 & $107.3(4)$ & C35 & C34 & C33 & $113.4(8)$ \\
\hline N13 & P4 & N15 & $98.9(4)$ & N5 & $\mathrm{C} 15$ & $\mathrm{C} 16$ & $111.0(7)$ \\
\hline N14 & P4 & N15 & $101.6(3)$ & N5 & C19 & $\mathrm{C} 18$ & $111.8(9)$ \\
\hline N12 & P4 & N13 & $114.4(4)$ & $\mathrm{C} 50$ & C49 & C48 & $111.7(8)$ \\
\hline N12 & $\mathrm{P} 4$ & N14 & $113.0(4)$ & $\mathrm{C} 41$ & $\mathrm{C} 42$ & $\mathrm{C} 43$ & $111.8(7)$ \\
\hline N12 & P4 & N15 & $119.8(4)$ & N11 & C46 & C47 & $111.5(7)$ \\
\hline N6 & $\mathrm{P} 2$ & N7 & $112.6(4)$ & C63 & C64 & C65 & $110.6(7)$ \\
\hline N6 & P2 & N8 & $120.6(4)$ & C44 & C43 & C42 & $110.3(7)$ \\
\hline N6 & P2 & C30 & $113.4(4)$ & C6 & C7 & $\mathrm{C} 8$ & 108.2(9) \\
\hline N7 & $\mathrm{P} 2$ & N8 & $99.2(4)$ & N15 & C61 & C62 & $110.4(6)$ \\
\hline N7 & P2 & C30 & $108.1(5)$ & C39 & C38 & C37 & $109.4(7)$ \\
\hline C30 & P2 & N8 & $101.3(4)$ & N9 & C36 & C37 & $112.0(9)$ \\
\hline $\mathrm{C} 1$ & $\mathrm{O} 1$ & $\mathrm{~K} 1$ & $128.6(7)$ & N10 & $\mathrm{C} 41$ & $\mathrm{C} 42$ & $110.7(8)$ \\
\hline $\mathrm{C} 3$ & $\mathrm{O} 1$ & K1 & $116.4(6)$ & $\mathrm{C} 32$ & $\mathrm{C} 31$ & $\mathrm{C} 30$ & $112.7(8)$ \\
\hline $\mathrm{C} 3$ & $\mathrm{O} 1$ & $\mathrm{C} 1$ & $113.9(8)$ & C38 & C37 & C36 & $110.7(7)$ \\
\hline C51 & N13 & P4 & $117.9(6)$ & C32 & C33 & C34 & $112.4(7)$ \\
\hline C55 & N13 & P4 & $125.5(6)$ & N14 & C56 & C57 & $113.7(10)$ \\
\hline C55 & N13 & $\mathrm{C} 51$ & $109.9(8)$ & N10 & $\mathrm{C} 45$ & $\mathrm{C} 44$ & $111.8(7)$ \\
\hline $\mathrm{C} 5$ & N3 & P1 & $111.5(6)$ & C9 & C8 & C7 & $112.5(9)$ \\
\hline C9 & N3 & P1 & 115.2(6) & N14 & C60 & C59 & $111.5(8)$ \\
\hline $\mathrm{C} 9$ & N3 & $\mathrm{C} 5$ & $109.3(7)$ & $\mathrm{C} 26$ & $\mathrm{C} 27$ & $\mathrm{C} 28$ & 110.1(9) \\
\hline C56 & N14 & P4 & $122.5(6)$ & C48 & $\mathrm{C} 47$ & $\mathrm{C} 46$ & $112.5(9)$ \\
\hline $\mathrm{C} 56$ & N14 & C60 & $112.6(7)$ & $\mathrm{C} 5$ & C6 & $\mathrm{C} 7$ & $111.2(8)$ \\
\hline C60 & N14 & P4 & $123.6(6)$ & N4 & $\mathrm{C} 10$ & $\mathrm{C} 11$ & $111.0(10)$ \\
\hline Tb1 & N6 & $\mathrm{K} 1$ & $74.23(19)$ & N8 & $\mathrm{C} 25$ & $\mathrm{C} 26$ & $110.3(9)$ \\
\hline P2 & N6 & Tb1 & $140.2(4)$ & $\mathrm{C} 31$ & $\mathrm{C} 30$ & P2 & $125.3(7)$ \\
\hline P2 & N6 & K1 & $122.7(3)$ & C31 & $\mathrm{C} 30$ & C35 & $110.9(8)$ \\
\hline $\mathrm{C} 20$ & N7 & $\mathrm{P} 2$ & $114.4(6)$ & $\mathrm{C} 35$ & $\mathrm{C} 30$ & $\mathrm{P} 2$ & $120.7(6)$ \\
\hline $\mathrm{C} 20$ & N7 & $\mathrm{C} 24$ & 110.2(9) & $\mathrm{C} 31$ & $\mathrm{C} 32$ & $\mathrm{C} 33$ & 113.1(9) \\
\hline $\mathrm{C} 24$ & N7 & P2 & $119.3(7)$ & $\mathrm{C} 15$ & $\mathrm{C} 16$ & $\mathrm{C} 17$ & $110.3(8)$ \\
\hline C36 & N9 & P3 & $125.6(5)$ & N3 & C5 & C6 & $111.3(8)$ \\
\hline C36 & N9 & $\mathrm{C} 40$ & $112.4(7)$ & N7 & $\mathrm{C} 20$ & $\mathrm{C} 21$ & $110.5(8)$ \\
\hline $\mathrm{C} 40$ & N9 & P3 & $122.0(7)$ & N13 & C51 & C52 & $111.2(8)$ \\
\hline C41 & N10 & P3 & $122.1(6)$ & $\mathrm{C} 27$ & $\mathrm{C} 26$ & $\mathrm{C} 25$ & $110.9(9)$ \\
\hline C45 & N10 & P3 & 114.1(5) & N13 & C55 & C54 & $110.3(7)$ \\
\hline $\mathrm{C} 45$ & N10 & $\mathrm{C} 41$ & $111.0(6)$ & C58 & C59 & C60 & $112.3(10)$ \\
\hline $\mathrm{C} 10$ & N4 & $\mathrm{P} 1$ & $126.1(7)$ & C55 & C54 & $\mathrm{C} 53$ & $111.5(9)$ \\
\hline $\mathrm{C} 10$ & N4 & $\mathrm{C} 14$ & $112.7(8)$ & $\mathrm{C} 29$ & $\mathrm{C} 28$ & $\mathrm{C} 27$ & $110.2(9)$ \\
\hline C14 & N4 & P1 & $120.9(6)$ & $\mathrm{C} 13$ & $\mathrm{C} 14$ & N4 & $113.4(10)$ \\
\hline C61 & N15 & P4 & $115.4(5)$ & N9 & $\mathrm{C} 40$ & C39 & $110.8(7)$ \\
\hline C65 & N15 & P4 & $114.8(5)$ & C56 & C57 & C58 & $110.8(8)$ \\
\hline
\end{tabular}




\begin{tabular}{llll}
\hline Atom & Atom & Atom & \multicolumn{1}{c}{ Angle $^{\circ}$} \\
\hline C52 & C53 & C54 & $107.7(10)$ \\
C14 & C13 & C12 & $111.1(12)$ \\
O1 & C1 & C2 & $109.6(9)$ \\
C34 & C35 & C30 & $110.5(9)$ \\
C10 & C11 & C12 & $110.6(10)$ \\
C45 & C44 & C43 & $111.5(9)$ \\
C3 & C4 & K1 & $86.8(7)$ \\
N3 & C9 & C8 & $110.4(8)$ \\
C51 & C52 & C53 & $112.3(8)$ \\
C24 & C23 & C22 & $114.1(9)$ \\
N8 & C29 & C28 & $110.2(8)$ \\
C23 & C24 & N7 & $109.1(9)$ \\
N11 & C50 & C49 & $109.4(8)$ \\
N15 & C65 & C64 & $109.8(7)$ \\
C64 & C63 & C62 & $110.7(7)$ \\
C19 & C18 & C17 & $109.9(8)$ \\
C20 & C21 & C22 & $108.5(9)$ \\
C59 & C58 & C57 & $111.5(8)$ \\
C47 & C48 & C49 & $108.2(7)$ \\
C61 & C62 & C63 & $111.3(7)$ \\
C11 & C12 & C13 & $111.2(10)$ \\
C23 & C22 & C21 & $108.9(11)$ \\
C18 & C17 & C16 & $108.8(7)$ \\
O1 & C3 & C4 & $110.0(9)$ \\
& & &
\end{tabular}

\begin{tabular}{|c|c|c|}
\hline Atom & Atom & Length/Å \\
\hline $\begin{array}{l}\text { P1 } 2 \\
\end{array}$ & N3 2 & $1.7034(12)$ \\
\hline $\mathrm{P} 1$ & $\mathrm{~N}_{4}^{-} 2$ & $1.6771(12)$ \\
\hline N2_2 & $\mathrm{C} 1 \_2$ & $1.4498(18)$ \\
\hline $\mathrm{N} 2 \_2$ & C7_2 & $1.4814(19)$ \\
\hline N3 2 & $\mathrm{C} 2-2$ & $1.4495(18)$ \\
\hline N3_2 & C3_2 & $1.4815(19)$ \\
\hline N4_-2 & $\mathrm{C} 1 \overline{2} 2$ & $1.4601(17)$ \\
\hline $\mathrm{N} 4-2$ & $\mathrm{C} 13^{-} 2$ & $1.4569(18)$ \\
\hline $\mathrm{C} 1 \_2$ & C2 $2 \overline{2}$ & $1.507(2)$ \\
\hline C3 3 & $\mathrm{C} 4 \_2$ & $1.536(2)$ \\
\hline C3_2 & C5_2 & $1.533(2)$ \\
\hline C3_2 & C6_2 & $1.524(2)$ \\
\hline $\mathrm{C} 72$ & $\mathrm{C} 82$ & $1.553(2)$ \\
\hline C7_2 & C9_2 & $1.524(2)$ \\
\hline $\mathrm{C}^{-}{ }^{2}$ & $\mathrm{C} 1 \overline{0} 2$ & $1.517(2)$ \\
\hline $\mathrm{C} 1 \overline{1} 2$ & $\mathrm{C} 12-2$ & $1.509(2)$ \\
\hline $\mathrm{C} 13 \_2$ & $\mathrm{C} 14 \_2$ & $1.523(3)$ \\
\hline O1_3 & C1_3 & $1.430(3)$ \\
\hline O1_3 & C3_3 & $1.399(9)$ \\
\hline C1_3 & $\mathrm{C} 2 \_3$ & $1.483(5)$ \\
\hline C3_3 & $\mathrm{C} 4 \_3$ & $1.496(5)$ \\
\hline
\end{tabular}

Table S7: Bond Lengths in $\AA$ for 3.

\begin{tabular}{lll}
\hline Atom & Atom & Length/Å \\
\hline Tb1 & K1 & $3.7858(5)$ \\
Tb1 & N1_1 & $2.2307(11)$ \\
Tb1 & N1_1 & $2.2307(11)$ \\
Tb1 & N1_2 & $2.2637(11)$ \\
Tb1 & N1_21 & $2.2637(10)$ \\
K1 & N1_21 & $2.8057(14)$ \\
K1 & N1_2 & $2.8058(14)$ \\
K1 & N2_21 & $3.1793(15)$ \\
K1 & O1_3 & $2.939(5)$ \\
P1_1 & N1_1 & $1.5283(11)$ \\
P1_1 & N2_1 & $1.7165(12)$ \\
P1_1 & N3_1 & $1.6955(12)$ \\
P1_1 & N4_1 & $1.6826(11)$ \\
N2_1 & C1_1 & $1.4537(18)$ \\
N2_1 & C7_1 & $1.4817(18)$ \\
N3_1 & C2_1 & $1.4463(18)$ \\
N3_1 & C3_1 & $1.4718(19)$ \\
N4_1 & C12_1 & $1.4568(17)$ \\
N4_1 & C13_1 & $1.4519(17)$ \\
C1_1 & C2_1 & $1.508(2)$ \\
C3_1 & C4_1 & $1.536(2)$ \\
C3_1 & C5_1 & $1.535(2)$ \\
C3_1 & C6_1 & $1.522(2)$ \\
C7_1 & C8_1 & $1.546(2)$ \\
C7_1 & C9 1 1 & $1.527(2)$ \\
C7_1 & C10_1 & $1.515(2)$ \\
C11_1 & C12_1 & $1.510(2)$ \\
C13_1 & C14_1 & $1.523(2)$ \\
P1_2 & N1_2 & $1.5318(11)$ \\
P1_2 & N2_2 & $1.7173(12)$ \\
& &
\end{tabular}


Table S8: Bond Angles in ${ }^{\circ}$ for 3.

\begin{tabular}{|c|c|c|c|}
\hline Atom & Atom & Atom & Angle $/^{\circ}$ \\
\hline$\overline{N 11} 1^{1}$ & $\mathrm{~Tb} 1$ & K1 & $125.39(3)$ \\
\hline N1_-1 & $\mathrm{Tb} 1$ & K1 & $125.39(3)$ \\
\hline N1_-1 & Tb1 & $\mathrm{N} 1 \_1^{1}$ & $109.22(7)$ \\
\hline N1_1 & Tb1 & N1_2 & $113.17(5)$ \\
\hline N1_-1 1 & Tb1 & N1_2 & $112.91(5)$ \\
\hline N1_-1 1 & $\mathrm{~Tb} 1$ & N1_2 ${ }^{1}$ & $113.18(5)$ \\
\hline N1_1 & $\mathrm{Tb} 1$ & N1_2 1 & $112.91(5)$ \\
\hline N1_2 & $\mathrm{Tb} 1$ & $\mathrm{~K} 1^{-}$ & $47.48(3)$ \\
\hline $\mathrm{N} 12^{1}$ & Tb1 & K1 & $47.48(3)$ \\
\hline N1_2 $2^{1}$ & Tb1 & N1_2 & $94.96(7)$ \\
\hline N1_2 $2^{1}$ & K1 & N1_2 & $72.97(4)$ \\
\hline $\mathrm{N} 12^{1}$ & K1 & $\mathrm{N} 2-2^{1}$ & $54.41(3)$ \\
\hline N1_2 & K1 & $\mathrm{N} 2{ }^{-} 2^{1}$ & $123.01(4)$ \\
\hline N1_2 & $\mathrm{K} 1$ & O1_3 & $144.2(2)$ \\
\hline N1_2 $2^{1}$ & K1 & $\mathrm{O}^{-}{ }_{-} 3$ & $142.3(2)$ \\
\hline $\mathrm{O} 1 \_3$ & K1 & $\mathrm{N} 2{ }^{-}{ }^{1}$ & $91.8(2)$ \\
\hline N1_-1 & $\mathrm{P} 1 \_1$ & $\mathrm{~N} 2-1$ & $120.75(7)$ \\
\hline N1_1 & $\mathrm{P} 1 \_1$ & N3_1 & $120.52(7)$ \\
\hline N1_1 & $\mathrm{P} 1 \_1$ & N4_1 & $113.38(7)$ \\
\hline N3_1 & $\mathrm{P} 1 \_1$ & N2_1 & $90.74(6)$ \\
\hline N4_1 & $\mathrm{P} 1-1$ & N2_1 & $103.72(6)$ \\
\hline N4_1 & $\mathrm{P} 1 \_1$ & N3_1 & $104.27(7)$ \\
\hline $\mathrm{P} 1 \_1$ & $\mathrm{~N} 1 \_1$ & $\mathrm{~Tb} \overline{1}$ & 168.78(9) \\
\hline $\mathrm{C} 1 \_1$ & $\mathrm{~N} 2-1$ & $\mathrm{P} 1 \_1$ & $109.65(10)$ \\
\hline $\mathrm{C} 1 \_1$ & $\mathrm{~N} 2 \_1$ & C7_1 & $117.06(12)$ \\
\hline $\mathrm{C}^{-}{ }_{-}^{-} 1$ & $\mathrm{~N} 2-1$ & $\mathrm{P} 1 \_-1$ & $123.22(9)$ \\
\hline $\mathrm{C} 2 \_1$ & N3_1 & $\mathrm{P} 1 \_1$ & $114.36(11)$ \\
\hline $\mathrm{C} 2 \_1$ & N3_1 & C3_1 & $117.61(12)$ \\
\hline C3_1 & N3_1 & $\mathrm{P} 1 \_1$ & $127.99(10)$ \\
\hline $\mathrm{C} 1 \overline{2} \_1$ & N4_1 & $\mathrm{P} 1 \_1$ & $120.77(10)$ \\
\hline C13_1 & N4_1 & P1_1 & $122.03(10)$ \\
\hline C13_1 & N4_1 & C12_1 & $117.06(12)$ \\
\hline N2_- & $\mathrm{Cl}_{-}^{-} 1$ & C2 $2 \overline{1}$ & $105.13(13)$ \\
\hline N3_1 & $\mathrm{C} 2 \_1$ & $\mathrm{C} 1 \_1$ & $105.86(12)$ \\
\hline N3_1 & C3_1 & $\mathrm{C} 4 \_1$ & $109.57(15)$ \\
\hline N3_1 & C3_1 & $\mathrm{C} 5$ & $109.08(14)$ \\
\hline N3_1 & C3_1 & C6_1 & $110.94(13)$ \\
\hline C5_1 & C3_1 & $\mathrm{C} 4 \_1$ & $109.51(15)$ \\
\hline C6_1 & C3_1 & $\mathrm{C} 4 \_1$ & $108.68(16)$ \\
\hline C6_1 & C3_1 & $\mathrm{C} 5$ & $109.03(16)$ \\
\hline N2_1 & C7_1 & C8_1 & $110.25(15)$ \\
\hline N2_-1 & $\mathrm{C}^{-}-1$ & C9_1 & $108.70(13)$ \\
\hline N2_1 & $\mathrm{C}^{-1} 1$ & C10_1 & $110.48(12)$ \\
\hline C9_1 & $\mathrm{C}^{-}-1$ & C8_- & $109.65(15)$ \\
\hline C10_1 & C7_1 & C8_1 & $108.02(15)$ \\
\hline C10_1 & C7_1 & C9_1 & $109.73(17)$ \\
\hline N4_1 & $\mathrm{C} 1 \overline{2} \_1$ & C11_1 & $115.72(15)$ \\
\hline N4_1 & C13_1 & C14_1 & $113.49(15)$ \\
\hline N1_2 & $\mathrm{P} 1 \_\overline{2}$ & $\mathrm{~K} 1{ }^{-}$ & $56.65(5)$ \\
\hline N1_2 & P1_2 & N2_2 & $115.93(7)$ \\
\hline N1_2 & $\mathrm{P} 1 \_2$ & N3_2 & $120.88(7)$ \\
\hline N1_2 & $\mathrm{P} 1 \_2$ & N4_2 & $113.79(7)$ \\
\hline N2_2 & $\mathrm{P} 1 \_2$ & $\mathrm{~K} 1^{-}$ & $69.63(5)$ \\
\hline N3_2 & $\mathrm{P} 1 \_2$ & $\mathrm{~K} 1$ & $92.42(5)$ \\
\hline N3_2 & $\mathrm{P} 1 \_2$ & N2_2 & $91.45(6)$ \\
\hline
\end{tabular}

\begin{tabular}{|c|c|c|c|}
\hline Atom & Atom & Atom & Angle $/^{\circ}$ \\
\hline$\overline{\mathrm{N} 4 \_2}$ & P1_2 & K1 & $163.94(5)$ \\
\hline N4_2 & $\mathrm{P} 1 \_2$ & $\mathrm{~N} 2 \_2$ & $108.47(7)$ \\
\hline N4_2 & $\mathrm{P} 1 \_2$ & N3_2 & $103.62(7)$ \\
\hline $\mathrm{Tb} \overline{1}$ & N1_2 & $\mathrm{K} 1{ }^{-}$ & $96.04(4)$ \\
\hline P1_2 & $\mathrm{N} 1 \_2$ & $\mathrm{~Tb} 1$ & $167.67(8)$ \\
\hline P1_2 & N1_2 & $\mathrm{K} 1$ & $96.22(6)$ \\
\hline $\mathrm{P} 1 \_2$ & $\mathrm{~N} 2 \_2$ & $\mathrm{~K} 1$ & $79.94(5)$ \\
\hline $\mathrm{C} 1 \_2$ & $\mathrm{~N} 2 \_2$ & K1 & $110.66(12)$ \\
\hline $\mathrm{C} 1 \_2$ & $\mathrm{~N} 2 \_2$ & $\mathrm{P} 1 \_2$ & $111.73(10)$ \\
\hline $\mathrm{C} 1 \_2$ & $\mathrm{~N} 2 \_2$ & $\mathrm{C} 72_{2}$ & $116.58(12)$ \\
\hline $\mathrm{C} 7 \_2$ & $\mathrm{~N} 2 \_2$ & $\mathrm{~K} 1$ & 104.70(9) \\
\hline $\mathrm{C} 7{ }^{-} 2$ & $\mathrm{~N} 2 \_2$ & $\mathrm{P} 1 \_2$ & $125.28(11)$ \\
\hline $\mathrm{C} 2 \_2$ & N3_2 & $\mathrm{P} 1 \_2$ & $113.51(11)$ \\
\hline $\mathrm{C} 2 \_2$ & N3_2 & C3_2 & $115.70(13)$ \\
\hline C3_2 & N3_2 & $\mathrm{P} 1 \_2$ & $123.55(10)$ \\
\hline $\mathrm{C} 1 \overline{2} \_2$ & $\mathrm{~N} 4 \_2$ & $\mathrm{P} 1 \_2$ & $122.67(11)$ \\
\hline C13_2 & N4_2 & P1_2 & $122.00(11)$ \\
\hline C13_2 & $\mathrm{N} 4 \_2$ & $\mathrm{C} 1 \overline{2} \_2$ & $115.34(14)$ \\
\hline N2_2 & $\mathrm{C} 1 \_2$ & C2_2 & $105.96(13)$ \\
\hline N3_2 & $\mathrm{C} 2 \_2$ & $\mathrm{C} 1 \_2$ & $106.16(13)$ \\
\hline N3_2 & C3_2 & $\mathrm{C} 4 \_2$ & $108.78(14)$ \\
\hline N3_2 & C3_2 & C5_2 & $110.17(15)$ \\
\hline N3_2 & C3_2 & C6_2 & $110.70(13)$ \\
\hline C5_2 & C3_2 & $\mathrm{C} 4 \_2$ & $109.58(17)$ \\
\hline C6_2 & C3_2 & $\mathrm{C} 4 \_2$ & $108.88(15)$ \\
\hline C6_2 & C3_2 & C5_2 & $108.71(15)$ \\
\hline $\mathrm{N} 2 \_2$ & C7_2 & C8_2 & $108.84(16)$ \\
\hline $\mathrm{N} 2 \_2$ & C7_2 & C9_2 & $111.35(14)$ \\
\hline N2_2 & C7_2 & C10_2 & $111.18(13)$ \\
\hline C9_2 & C7_2 & C8_2 & $108.63(17)$ \\
\hline C10_2 & C7_2 & C8_2 & $106.75(17)$ \\
\hline C10_2 & C7_2 & C9_2 & $109.95(18)$ \\
\hline N4_2 & C12_2 & C11_2 & $115.52(16)$ \\
\hline N4_2 & C13_2 & C14_2 & $113.72(19)$ \\
\hline C1_3 & O1_3 & $\mathrm{K} 1$ & $133.1(5)$ \\
\hline C3_3 & O1_3 & K1 & $108.3(4)$ \\
\hline C3_3 & $\mathrm{O} 1{ }^{-} 3$ & C1_3 & $114.9(6)$ \\
\hline O1_3 & C1_3 & C2_3 & $104.1(4)$ \\
\hline O1_3 & C3_3 & C4_3 & $122.9(11)$ \\
\hline
\end{tabular}


Table S9: Bond Lengths in $\AA$ for 3-100K.

\begin{tabular}{|c|c|c|}
\hline Atom & Atom & Length/Å \\
\hline$\overline{\mathrm{Tb} 1}$ & N1_5 & $2.239(5)$ \\
\hline Tb1 & N1_6 & $2.256(5)$ \\
\hline $\mathrm{Tb} 1$ & N1_7 & $2.285(5)$ \\
\hline $\mathrm{Tb} 1$ & N1_8 & $2.235(5)$ \\
\hline K1 & O1S__1 & $3.146(6)$ \\
\hline K1 & O1S_2 & $3.148(6)$ \\
\hline K1 & N1_6 & $2.829(5)$ \\
\hline K1 & N6_6 & $3.098(6)$ \\
\hline K1 & N1_7 & $2.749(5)$ \\
\hline K1 & N6_7 & $3.208(6)$ \\
\hline O1S_1 & C2S__1 & $1.402(6)$ \\
\hline O1S_1 & $\mathrm{C} 4 \mathrm{~S}_{-}{ }^{-1}$ & $1.402(6)$ \\
\hline C2S_1 & C3S_1 & $1.505(10)$ \\
\hline C4S_1 & C5S_1 & $1.484(11)$ \\
\hline O1S_2 & C2S_2 & $1.402(6)$ \\
\hline O1S_2 & C4S_2 & $1.403(6)$ \\
\hline C2S_2 & C3S_2 & $1.504(10)$ \\
\hline C4S_2 & C5S_2 & $1.484(11)$ \\
\hline O1S_3 & C2S_3 & $1.402(6)$ \\
\hline O1S_3 & $\mathrm{C} 4 \mathrm{~S} \_3$ & $1.402(6)$ \\
\hline C2S_3 & C3S_3 & $1.504(10)$ \\
\hline C4S_3 & C5S_3 & $1.484(11)$ \\
\hline O1S_4 & C2S_4 & $1.402(6)$ \\
\hline O1S_4 & C4S_4 & $1.402(6)$ \\
\hline $\mathrm{C} 2 \mathrm{~S}_{-} 4$ & C3S_4 & $1.504(10)$ \\
\hline $\mathrm{C} 4 \mathrm{~S}_{-}^{-} 4$ & $\mathrm{C}^{2} \mathrm{~S}_{-}^{-} 4$ & $1.484(11)$ \\
\hline $\mathrm{P} 1 \_\overline{5}$ & N1_ 5 & $1.530(5)$ \\
\hline $\mathrm{P} 1 \_5$ & N5_5 & $1.680(5)$ \\
\hline $\mathrm{P} 1 \_5$ & N6_5 & $1.726(5)$ \\
\hline P1_5 & N7_5 & $1.687(5)$ \\
\hline N5_5 & C12_5 & $1.460(7)$ \\
\hline N5_5 & C13_5 & $1.455(8)$ \\
\hline N6_5 & C1_ $\overline{5}$ & $1.462(8)$ \\
\hline N6_5 & C7_5 & $1.483(8)$ \\
\hline N7_5 & $\mathrm{C} 2 \_5$ & $1.454(7)$ \\
\hline N7_5 & C3_5 & $1.483(8)$ \\
\hline $\mathrm{C} 1 \_5$ & $\mathrm{C} 2-5$ & $1.508(9)$ \\
\hline C3_5 & C4 5 & $1.530(9)$ \\
\hline C3_5 & C5_5 & $1.538(9)$ \\
\hline C3_5 & C6_5 & $1.527(9)$ \\
\hline C7_5 & C8_5 & $1.534(9)$ \\
\hline $\mathrm{C}^{-}{ }^{-} 5$ & C9_5 & $1.544(9)$ \\
\hline C7_5 & C10_5 & $1.535(9)$ \\
\hline C11_5 & C12_5 & $1.540(9)$ \\
\hline C13_5 & C14_5 & $1.501(9)$ \\
\hline P1_6 & N1_6 & $1.530(5)$ \\
\hline P1_6 & N5_6 & $1.687(5)$ \\
\hline P1_6 & N6_6 & $1.743(5)$ \\
\hline P1_6 & N7_6 & $1.707(5)$ \\
\hline N5_6 & C12_6 & $1.460(8)$ \\
\hline N5_6 & C13_6 & $1.469(7)$ \\
\hline N6_6 & C1_6 & $1.469(8)$ \\
\hline N6_6 & C7_6 & $1.463(8)$ \\
\hline N7_6 & C2_6 & $1.464(7)$ \\
\hline N7_6 & C3_6 & $1.466(8)$ \\
\hline
\end{tabular}

\begin{tabular}{|c|c|c|}
\hline Atom & Atom & Length/Å \\
\hline$\overline{\mathrm{C} 1 \_6}$ & C2_6 & $1.500(10)$ \\
\hline $\mathrm{C} 36$ & $\mathrm{C} 4-6$ & $1.537(8)$ \\
\hline $\mathrm{C} 3^{-} 6$ & $\mathrm{C} 5-6$ & $1.543(9)$ \\
\hline $\mathrm{C} 3{ }_{-}^{-} 6$ & C6_6 & $1.537(9)$ \\
\hline C7_6 & C8_6 & $1.536(9)$ \\
\hline C7_6 & C9_6 & $1.531(9)$ \\
\hline C7_6 & C10_6 & $1.528(8)$ \\
\hline $\mathrm{C} 1 \overline{1} \_6$ & $\mathrm{C} 12-6$ & $1.523(9)$ \\
\hline C13_6 & C14_6 & $1.532(9)$ \\
\hline P1 $\overline{7}$ & $\mathrm{~N} 1 \overline{7}$ & $1.526(5)$ \\
\hline $\mathrm{P} 1{ }^{-} 7$ & $\mathrm{~N} 5^{-} 7$ & $1.678(6)$ \\
\hline P1_7 & N6_7 & $1.738(5)$ \\
\hline P1_7 & N7_7 & $1.701(5)$ \\
\hline N5_7 & $\mathrm{C} 1 \overline{2} \_7$ & $1.460(8)$ \\
\hline N5 7 & $\mathrm{C} 13-7$ & $1.464(9)$ \\
\hline N6_7 & $\mathrm{C} 1 \_\overline{7}$ & $1.458(8)$ \\
\hline N6_7 & C7_7 & $1.482(8)$ \\
\hline N7 7 & $\mathrm{C} 22^{-} 7$ & $1.468(8)$ \\
\hline N7 7 & $\mathrm{C} 37$ & $1.474(8)$ \\
\hline $\mathrm{C} 1 \_7$ & $\mathrm{C} 2 \_7$ & $1.504(10)$ \\
\hline C3_7 & C4_7 & $1.523(9)$ \\
\hline C3_7 & C5_7 & $1.533(10)$ \\
\hline $\mathrm{C} 37$ & C6 7 & $1.535(8)$ \\
\hline C7_7 & C8_7 & $1.539(10)$ \\
\hline C7_-7 & $\mathrm{C} 9-7$ & $1.526(10)$ \\
\hline $\mathrm{C} 7^{-} 7$ & $\mathrm{C} 1 \overline{0} 7$ & $1.518(10)$ \\
\hline C11_7 & $\mathrm{C} 12 \_7$ & $1.524(11)$ \\
\hline C13_7 & C14_7 & $1.527(10)$ \\
\hline P1_ $\overline{8}$ & N1_ $\_$ & $1.526(5)$ \\
\hline $\mathrm{P} 1 \_8$ & $\mathrm{~N} 58$ & $1.698(5)$ \\
\hline P1_8 & N6_8 & $1.710(5)$ \\
\hline P1_8 & N7_8 & $1.707(5)$ \\
\hline N5_8 & $\mathrm{C} 1 \overline{2} \_8$ & $1.452(8)$ \\
\hline $\mathrm{N} 58$ & $\mathrm{C} 13{ }_{-}^{-}$ & $1.462(8)$ \\
\hline N6_8 & $\mathrm{C} 1 \_\overline{8}$ & $1.461(7)$ \\
\hline N6_8 & C7_8 & $1.492(7)$ \\
\hline N7_8 & $\mathrm{C} 2 \_8$ & $1.442(8)$ \\
\hline N7_-8 & $\mathrm{C} 3$ & $1.474(8)$ \\
\hline C1_8 & $\mathrm{C} 2 \_8$ & $1.513(9)$ \\
\hline $\mathrm{C} 3 \_8$ & $\mathrm{C} 4 \_8$ & $1.536(11)$ \\
\hline $\mathrm{C} 38$ & $\mathrm{C} 58$ & $1.535(11)$ \\
\hline $\mathrm{C} 3{ }_{-}^{-}$ & C6 8 & $1.513(10)$ \\
\hline C7_8 & C8_8 & $1.535(9)$ \\
\hline C7_8 & C9_8 & $1.537(9)$ \\
\hline $\mathrm{C}^{-} 8$ & $\mathrm{C} 1 \overline{0} 8$ & $1.535(9)$ \\
\hline C1표 8 & $\mathrm{C} 12-8$ & $1.524(11)$ \\
\hline C13_8 & C14_8 & $1.531(10)$ \\
\hline
\end{tabular}


Table S10: Bond Angles in ${ }^{\circ}$ for $\mathbf{3 - 1 0 0 K}$.

\begin{tabular}{|c|c|c|c|}
\hline Atom & Atom & Atom & Angle $/^{\circ}$ \\
\hline N1_5 & Tb1 & N1_6 & $112.39(18)$ \\
\hline N1_5 & Tb1 & N1_7 & $114.23(17)$ \\
\hline N1_6 & $\mathrm{Tb} 1$ & N1_7 & $94.61(18)$ \\
\hline N1_8 & $\mathrm{Tb} 1$ & N1_5 & $110.25(18)$ \\
\hline N1_8 & Tb1 & N1_6 & $112.15(18)$ \\
\hline N1_8 & Tb1 & N1_7 & $112.46(19)$ \\
\hline O1) & K1 & N6_7 & $89.4(2)$ \\
\hline O1S_2 & K1 & N6_7 7 & $96.8(3)$ \\
\hline N1_ $\overline{6}$ & K1 & O1S_1 & $132.70(19)$ \\
\hline N1_6 & K1 & O1S_2 & $128.8(2)$ \\
\hline N1_6 6 & K1 & N6_ 6 & $55.35(14)$ \\
\hline N1_6 & K1 & N6_-7 & $125.91(14)$ \\
\hline N6_6 & K1 & O1S_1 & $96.7(2)$ \\
\hline N6_6 & K1 & O1S_2 & $88.8(3)$ \\
\hline N6_6 & K1 & N6_ $\overline{7}$ & $168.25(14)$ \\
\hline N1_-7 & K1 & $\mathrm{O} 1 \overline{\mathrm{S}} \_1$ & $141.1(2)$ \\
\hline N1_7 & K1 & O1S_2 & $149.3(3)$ \\
\hline N1_7 & $\mathrm{K} 1$ & N1_6 & $73.49(15)$ \\
\hline N1_7 & K1 & N6_6 & $121.46(14)$ \\
\hline N1_7 & K1 & N6_-7 & $54.47(14)$ \\
\hline $\mathrm{C} 2 \overline{\mathrm{S}} \_1$ & O1S_1 & $\mathrm{K} 1{ }^{-}$ & $111.3(5)$ \\
\hline $\mathrm{C} 2 \mathrm{~S}_{-}^{-} 1$ & O1S_1 & C4S_1 & $112.7(6)$ \\
\hline C4S_1 & O1S_1 & $\mathrm{K} 1$ & $133.6(5)$ \\
\hline O1S_1 & $\mathrm{C} 2 \mathrm{~S}_{-}^{-} 1$ & C3S_1 & $110.5(6)$ \\
\hline $\mathrm{C} 2 \mathrm{~S}_{-}^{-} 1$ & C3S_-1 & $\mathrm{K} 1{ }^{-}$ & $95.3(5)$ \\
\hline O1S_1 & C4S_1 & C5S_1 & $111.8(6)$ \\
\hline C2S_2 & O1S_2 & $\mathrm{K} 1$ & $108.7(6)$ \\
\hline C2S_2 & O1S_2 & C4S_2 & $112.6(6)$ \\
\hline $\mathrm{C} 4 \mathrm{~S}^{-} 2$ & O1S_2 & $\mathrm{K} 1{ }^{-}$ & $138.6(7)$ \\
\hline O1S_2 & C2S_2 & C3S_2 & $110.5(6)$ \\
\hline $\mathrm{C} 2 \mathrm{~S} \_2$ & C3S_2 & $\mathrm{K} 1{ }^{-}$ & $97.7(5)$ \\
\hline O1S_2 & C4S_2 & C5S_2 & $111.8(7)$ \\
\hline $\mathrm{C} 2 \mathrm{~S} \_3$ & O1S_3 & C4S_3 & $112.7(6)$ \\
\hline O1S_3 & C2S_3 & C3S_3 & $110.5(6)$ \\
\hline O1S_3 & C4S_3 & C5S_3 & $111.8(7)$ \\
\hline $\mathrm{C} 2 \mathrm{~S} \_4$ & $\mathrm{O} 1 \mathrm{~S} \_4$ & $\mathrm{C} 4 \mathrm{~S}_{-}-4$ & $112.6(6)$ \\
\hline O1S_4 & $\mathrm{C} 2 \mathrm{~S}_{-}^{-} 4$ & C3S_4 & $110.5(6)$ \\
\hline O1S_4 & $\mathrm{C} 4 \mathrm{~S}_{-}^{-} 4$ & $\mathrm{C} \mathrm{S}_{-}^{-} 4$ & $111.8(7)$ \\
\hline N1_ $\overline{5}$ & $\mathrm{P} 1 \_\overline{5}$ & N5_ $\overline{5}$ & $113.5(3)$ \\
\hline N1_5 & $\mathrm{P} 1 \_5$ & N6_5 & $120.7(3)$ \\
\hline N1_5 & $\mathrm{P} 1 \_5$ & N7_5 & $121.0(3)$ \\
\hline N5_5 & $\mathrm{P} 1 \_5$ & N6_5 & $104.2(2)$ \\
\hline N5_5 & $\mathrm{P} 1 \_5$ & N7_5 & $103.6(3)$ \\
\hline N7_5 & $\mathrm{P} 1 \_5$ & N6_5 & $90.4(2)$ \\
\hline $\mathrm{P} 1 \_5$ & N1_5 & $\mathrm{Tb} \overline{1}$ & $172.5(3)$ \\
\hline C12_5 & N5_5 & P1_5 & $121.2(4)$ \\
\hline C13_5 & N5_5 & $\mathrm{P} 1 \_5$ & $122.3(4)$ \\
\hline $\mathrm{C} 13{ }^{-} 5$ & N5_5 & C12_5 & $116.3(5)$ \\
\hline $\mathrm{C} 1 \_\overline{5}$ & N6_5 & $\mathrm{P} 1 \_\overline{5}$ & $109.2(4)$ \\
\hline $\mathrm{C} 1 \_5$ & N6_5 & $\mathrm{C} 7{ }_{-}^{-5}$ & $116.4(5)$ \\
\hline C7_5 & N6_5 & $\mathrm{P} 1 \_5$ & $122.0(4)$ \\
\hline $\mathrm{C} 2 \_5$ & N7_5 & $\mathrm{P} 1 \_5$ & $114.4(4)$ \\
\hline $\mathrm{C} 2 \_5$ & N7_5 & $\mathrm{C} 3{ }^{-} 5$ & $118.1(5)$ \\
\hline C3_5 & N7_5 & $\mathrm{P} 1 \_5$ & $127.3(4)$ \\
\hline N6_5 & $\mathrm{Cl}^{-} 5$ & $\mathrm{C} 2 \_5$ & $104.2(5)$ \\
\hline N7_5 & $\mathrm{C} 2-5$ & $\mathrm{Cl}_{-}^{-} 5$ & $106.1(5)$ \\
\hline N7_5 & C3_5 & $\mathrm{C} 4{ }^{-} 5$ & $109.6(5)$ \\
\hline
\end{tabular}

\begin{tabular}{|c|c|c|c|}
\hline Atom & Atom & Atom & Angle $/^{\circ}$ \\
\hline N7_5 & C3_5 & C5_5 & $108.8(5)$ \\
\hline N7_5 & C3_5 & C6_5 & $110.7(5)$ \\
\hline $\mathrm{C}^{-} 5$ & C3 35 & C5 5 & $109.4(5)$ \\
\hline C6_5 & C3_5 & $\mathrm{C} 4{ }^{-} 5$ & $108.7(5)$ \\
\hline C6_5 & C3 35 & C5 5 & $109.6(5)$ \\
\hline N6_5 & C7 ${ }^{-5}$ & C8_5 & $111.2(5)$ \\
\hline N6_5 & $\mathrm{C}^{-}{ }_{-}^{5}$ & C9-5 & $107.6(5)$ \\
\hline N6_5 & $\mathrm{C}^{-}{ }_{-}^{-5}$ & $\mathrm{C} 1 \overline{0} 5$ & $109.9(5)$ \\
\hline C8_5 & $\mathrm{C}^{-}{ }^{-5}$ & C9 $\overline{5}$ & $110.2(5)$ \\
\hline $\mathrm{C} 8{ }^{-} 5$ & $\mathrm{C}^{-}{ }^{-5}$ & $\mathrm{C} 1 \overline{0} 5$ & $109.2(5)$ \\
\hline $\mathrm{C} 1 \overline{0} 55$ & $\mathrm{C} 7{ }^{-} 5$ & C9 $\overline{5}$ & $108.6(5)$ \\
\hline N5 $\overline{5}$ & $\mathrm{C} 1 \overline{2} \_5$ & C1ㄴ_s 5 & $114.8(5)$ \\
\hline N5_5 & C13_5 & C14_5 & $115.1(6)$ \\
\hline N1_6 6 & P1_ $\overline{6}$ & N5_ $\overline{6}$ & $113.5(3)$ \\
\hline N1_6 6 & $\mathrm{P} 1 \_6$ & N6_6 & $115.0(3)$ \\
\hline N1_6 6 & $\mathrm{P} 1 \_6$ & N7_6 & $121.0(3)$ \\
\hline N5_6 & $\mathrm{P} 1{ }^{-} 6$ & N6_6 & $110.7(3)$ \\
\hline N5_6 & $\mathrm{P} 1 \_6$ & N7_6 & $102.8(3)$ \\
\hline N7 ${ }^{-} 6$ & $\mathrm{P} 1{ }^{-} 6$ & N6_6 6 & $91.5(2)$ \\
\hline $\mathrm{Tb} \overline{1}$ & N1_6 & $\mathrm{K} 1$ & $95.06(17)$ \\
\hline P1_6 & N1_6 & $\mathrm{Tb} 1$ & $171.6(3)$ \\
\hline P1_6 & N1_6 & K1 & $93.0(2)$ \\
\hline C12_6 & N5_6 & P1_6 & $123.0(4)$ \\
\hline C12_6 & N5_6 & C15_6 & $116.0(5)$ \\
\hline C13_6 & N5_6 & $\mathrm{P} 1 \_\overline{6}$ & $120.9(4)$ \\
\hline P1_ 6 & N6_6 & $\mathrm{K} 1^{-}$ & $80.21(19)$ \\
\hline C1_6 & N6_6 & K1 & $112.1(4)$ \\
\hline $\mathrm{C} 1 \_6$ & N6_6 & P1_6 & $109.2(4)$ \\
\hline C7_6 & N6_6 & $\mathrm{K} 1$ & 109.7(3) \\
\hline C7_6 6 & N6_6 & P1_6 & $124.5(4)$ \\
\hline C7_6 6 & N6_6 & $\mathrm{Cl}^{-}{ }_{-} 6$ & $115.6(5)$ \\
\hline $\mathrm{C} 2 \_6$ & N7_6 & P1_6 & $113.0(4)$ \\
\hline $\mathrm{C} 2 \_6$ & N7_6 & C3_6 & $116.5(5)$ \\
\hline C3_6 & N7_6 & P1_6 & $122.3(4)$ \\
\hline N6_6 & C1_6 & $\mathrm{C} 2 \_6$ & $105.2(5)$ \\
\hline N7_6 6 & $\mathrm{C} 2{ }^{-} 6$ & $\mathrm{Cl}^{-}{ }^{-} 6$ & $106.0(5)$ \\
\hline N7_6 & C3_6 & C4_6 & $110.1(5)$ \\
\hline N7_6 & C3_6 & C5_6 & $112.5(5)$ \\
\hline N7-6 & C3_6 & C6_6 & $110.0(5)$ \\
\hline C4_6 & C3_6 & C5_6 & $108.1(6)$ \\
\hline C4_6 & C3_6 & C6_6 & $108.0(5)$ \\
\hline C6_6 6 & C3_6 & C5_6 & $108.1(6)$ \\
\hline C3_6 & C5_6 & $\mathrm{K}^{-}$ & $108.2(4)$ \\
\hline N6_6 & C7-6 & C8_6 & $109.0(5)$ \\
\hline N6_6 & C7-6 & C9_6 & $112.4(5)$ \\
\hline N6_6 & C7_6 & C10_6 & $110.8(5)$ \\
\hline C9_6 & C7_6 & C8_ 6 & $109.2(5)$ \\
\hline C10_6 & C7-6 & C8_6 & $106.8(5)$ \\
\hline C10_6 & C7_6 & C9_6 & $108.5(6)$ \\
\hline N5_6 & C12_6 & C1ㅎ﹎6 & $114.2(6)$ \\
\hline N5_6 & C13_6 & C14_6 & $113.1(5)$ \\
\hline N1_7 & $\mathrm{P} 1 \_\overline{7}$ & N5_ $\overline{7}$ & $113.7(3)$ \\
\hline N1_7 & $\mathrm{P} 1{ }^{-} 7$ & N6_7 7 & $115.1(3)$ \\
\hline N1_7 & $\mathrm{P} 1 \_7$ & N7_7 & $121.9(3)$ \\
\hline N5_7 & $\mathrm{P} 1{ }^{-} 7$ & N6_7 & $109.4(3)$ \\
\hline N5_7 & $\mathrm{P} 1 \_7$ & N7 7 & $102.8(3)$ \\
\hline N7 ${ }_{-7} 7$ & $\mathrm{P} 1-7$ & N6_7 7 & $91.4(3)$ \\
\hline $\mathrm{Tb} \overline{1}$ & N1_7 & $\mathrm{K}^{-}$ & $96.57(17)$ \\
\hline P1_7 & N1_7 & $\mathrm{Tb} 1$ & $162.8(3)$ \\
\hline
\end{tabular}




\begin{tabular}{|c|c|c|c|}
\hline Atom & Atom & Atom & Angle $/^{\circ}$ \\
\hline P1_7 & N1_7 & K1 & $100.6(2)$ \\
\hline $\mathrm{C} 1 \overline{2} 7$ & $\mathrm{~N} 5^{-} 7$ & P1 7 & $122.8(5)$ \\
\hline $\mathrm{C} 12-7$ & N5 7 & $\mathrm{C} 1 \overline{3} \_7$ & $115.8(5)$ \\
\hline C13_7 & N5_7 & P1_7 & $121.4(4)$ \\
\hline P1_7 & N6_7 & K1 & $80.30(18)$ \\
\hline $\mathrm{Cl}^{--} 7$ & N6 7 & $\mathrm{~K} 1$ & $117.6(4)$ \\
\hline $\mathrm{Cl}^{-}{ }_{-7}$ & N6_7 & P1_7 & $110.8(4)$ \\
\hline C1_7 & N6_7 & C7_7 & 115.3(5) \\
\hline $\mathrm{C} 7{ }_{-7}$ & N6_7 & $\mathrm{K} 1^{-}$ & $103.0(3)$ \\
\hline $\mathrm{C} 7^{-} 7$ & N6 7 & P1 7 & $124.6(4)$ \\
\hline $\mathrm{C} 2-7$ & N7_7 & $\mathrm{P}_{1-7}^{-} 7$ & $113.5(4)$ \\
\hline $\mathrm{C} 2 \_7$ & N7_7 & C3_7 & $115.9(5)$ \\
\hline C3_7 & N7_7 & P1_7 & 124.2(4) \\
\hline N6-7 & $\mathrm{C} 1_{-7}^{-7}$ & $\mathrm{C} 2-7$ & $105.9(5)$ \\
\hline N7_7 & $\mathrm{C} 2-7$ & $\mathrm{C} 1 \_7$ & $105.5(6)$ \\
\hline N7-7 & $\mathrm{C} 3-7$ & $\mathrm{C} 4 \_7$ & $109.0(5)$ \\
\hline N7 7 & $\mathrm{C} 3^{-} 7$ & $\mathrm{C} 5^{-} 7$ & $109.8(6)$ \\
\hline N7-7 & $\mathrm{C} 3-7$ & C6_7 & $110.5(5)$ \\
\hline C4_7 & C3_7 & C5_7 & $110.2(7)$ \\
\hline C4_7 & C3_7 & C6_7 & $108.9(6)$ \\
\hline C5_7 & C3_7 & C6_7 & $108.4(5)$ \\
\hline N6_7 & $\mathrm{C} 7{ }_{-7}^{-7}$ & C8 7 & $108.2(6)$ \\
\hline N6_7 & C7_7 & C9_7 & $112.7(6)$ \\
\hline N6_7 & $\mathrm{C} 7{ }_{-7}^{-7}$ & $\mathrm{C} 1 \overline{0} 7$ & $110.3(5)$ \\
\hline $\mathrm{C} 9^{-} 7$ & $\mathrm{C}^{-}{ }^{-7}$ & $\mathrm{C} 8 \overline{7}$ & $109.9(6)$ \\
\hline $\mathrm{C} 10 \_7$ & $\mathrm{C} 7{ }_{-}^{-7}$ & C8 8 & $107.2(6)$ \\
\hline C10_7 & C7_7 & C9_7 & $108.5(7)$ \\
\hline N5_7 & C12_7 & C11_7 & $114.7(6)$ \\
\hline $\mathrm{N} 57$ & $\mathrm{C} 13^{-7} 7$ & $\mathrm{C} 14 \_7$ & $113.5(6)$ \\
\hline N1_8 & P1_z & N5_ $\overline{8}$ & $113.2(3)$ \\
\hline N1_8 & P1_8 & N6_8 & $121.6(3)$ \\
\hline N1_8 & P1_8 & N7_8 & $119.8(3)$ \\
\hline $\mathrm{N} 58$ & $\mathrm{P} 1-8$ & N6_8 & $103.1(3)$ \\
\hline N5_8 & P1_8 & N7_8 & $104.7(3)$ \\
\hline N7_8 & P1_8 & N6_8 & $91.1(3)$ \\
\hline P1_8 & N1_8 & Tb1 & $167.6(3)$ \\
\hline $\mathrm{C} 1 \overline{2} \_8$ & N5_8 & P1_8 & $121.2(4)$ \\
\hline C12_8 & N5_8 & C13_8 & $117.4(5)$ \\
\hline C13_8 & N5_8 & P1_8 & $121.0(4)$ \\
\hline $\mathrm{C} 1 \_\overline{8}$ & N6 8 & $\mathrm{P}^{-} 8$ & $110.1(4)$ \\
\hline $\mathrm{Cl} 18$ & N6_8 & C7_8 & $115.2(5)$ \\
\hline C7_8 & N6_8 & P1_8 & $122.8(4)$ \\
\hline C2_8 & N7_8 & P1_8 & $113.8(4)$ \\
\hline $\mathrm{C} 2-8$ & N7 8 & $\mathrm{C} 3-8$ & $118.0(5)$ \\
\hline $\mathrm{C} 38$ & N7_8 & $\mathrm{P} 1 \_8$ & $127.2(4)$ \\
\hline N6_8 & C1_8 & $\mathrm{C} 2 \_8$ & $104.7(5)$ \\
\hline N7_8 & $\mathrm{C} 2 \_8$ & $\mathrm{C} 1 \_8$ & $105.9(5)$ \\
\hline N7 8 & $\mathrm{C} 3-8$ & $\mathrm{C}^{-}-8$ & $109.8(6)$ \\
\hline N7_8 & C3_8 & $\mathrm{C} 5 \_8$ & $108.8(6)$ \\
\hline N7_8 & C3_8 & C6_8 & $110.9(5)$ \\
\hline C5_8 & C3_8 & C4_8 & $108.5(7)$ \\
\hline $\mathrm{C} 68$ & $\mathrm{C} 3{ }_{8} 8$ & $\mathrm{C} 4 \_8$ & $109.8(7)$ \\
\hline C6_8 & C3_8 & C5_8 & $109.0(7)$ \\
\hline N6_8 & C7_8 & C8_8 & $112.5(5)$ \\
\hline $\mathrm{N}^{-} 8$ & $\mathrm{C}^{-} 8$ & $\mathrm{C} 9{ }^{-} 8$ & $108.0(5)$ \\
\hline N6_8 & $\mathrm{C} 7 \_8$ & $\mathrm{C} 1 \overline{0} 8$ & 109.1(5) \\
\hline $\mathrm{C} 8 \_8$ & C7_8 & C9_8 & $110.3(5)$ \\
\hline C8_8 & C7_8 & C10_8 & $108.2(5)$ \\
\hline C10_8 & C7_8 & C9_8 & $108.8(5)$ \\
\hline
\end{tabular}

\begin{tabular}{lllc}
\hline Atom & Atom & Atom & Angle $^{\circ}$ \\
\hline N5_8 & C12_8 & C11_8 & $115.6(6)$ \\
N5_8 & C13_8 & C14_8 & $112.8(6)$
\end{tabular}


Table S11: Bond Lengths in $\AA$ for 4.

\begin{tabular}{|c|c|c|}
\hline Atom & Atom & Length/Å \\
\hline Tb1 & N1 & $2.106(3)$ \\
\hline Tb1 & $\mathrm{N} 1^{1}$ & $2.106(3)$ \\
\hline Tb1 & $\mathrm{N} 1^{2}$ & $2.106(3)$ \\
\hline Tb1 & $\mathrm{N} 1^{3}$ & $2.106(3)$ \\
\hline P1 & N3 & $1.680(3)$ \\
\hline P1 & $\mathrm{N} 2$ & $1.697(4)$ \\
\hline $\mathrm{P} 1$ & N4 & $1.667(4)$ \\
\hline P1 & N1 & $1.555(3)$ \\
\hline N3 & C7 & $1.482(5)$ \\
\hline N3 & $\mathrm{C} 2$ & $1.456(5)$ \\
\hline N2 & $\mathrm{C} 3$ & $1.493(6)$ \\
\hline N2 & $\mathrm{C} 1$ & $1.469(6)$ \\
\hline N4 & C11 & $1.462(7)$ \\
\hline N4 & $\mathrm{C} 13$ & $1.468(5)$ \\
\hline C11 & $\mathrm{C} 12$ & $1.529(6)$ \\
\hline C3 & $\mathrm{C} 4$ & $1.532(6)$ \\
\hline $\mathrm{C} 3$ & $\mathrm{C} 5$ & $1.538(6)$ \\
\hline $\mathrm{C} 3$ & C6 & $1.523(7)$ \\
\hline C7 & $\mathrm{C} 8$ & $1.548(9)$ \\
\hline C7 & C9 & $1.539(6)$ \\
\hline C7 & $\mathrm{C} 10$ & $1.530(6)$ \\
\hline $\mathrm{C} 1$ & $\mathrm{C} 2$ & $1.517(6)$ \\
\hline C14 & C13 & $1.525(6)$ \\
\hline $\mathrm{C} 1 \mathrm{~S} 1$ & $\mathrm{C} 2 \mathrm{~S} 1$ & $1.518(3)$ \\
\hline $\mathrm{C} 2 \mathrm{~S}^{-} 1$ & $\mathrm{C} 3 \mathrm{~S}^{-} 1$ & $1.519(3)$ \\
\hline $\mathrm{C} 3 \mathrm{~S}_{-}^{-1}$ & C4S_-1 & $1.518(3)$ \\
\hline C4S_1 & C5S_1 & $1.518(3)$ \\
\hline
\end{tabular}


Table S12: Bond Angles in ${ }^{\circ}$ for 4.

\begin{tabular}{|c|c|c|c|}
\hline Atom & Atom & Atom & Angle $/^{\circ}$ \\
\hline N1 & Tb1 & $\mathrm{N} 1^{1}$ & $108.26(7)$ \\
\hline $\mathrm{N} 1^{2}$ & $\mathrm{~Tb} 1$ & $\mathrm{~N} 1^{3}$ & $108.26(7)$ \\
\hline N1 & $\mathrm{Tb} 1$ & $\mathrm{~N} 1^{3}$ & $111.91(14)$ \\
\hline N1 & $\mathrm{Tb} 1$ & $\mathrm{~N}^{2}$ & $108.26(7)$ \\
\hline $\mathrm{N} 1^{1}$ & $\mathrm{~Tb} 1$ & $\mathrm{~N} 1^{2}$ & $111.91(14)$ \\
\hline $\mathrm{N} 1^{1}$ & Tb1 & $\mathrm{N} 1^{3}$ & $108.26(7)$ \\
\hline N3 & P1 & N2 & $91.58(19)$ \\
\hline N4 & $\mathrm{P} 1$ & N3 & $105.60(19)$ \\
\hline N4 & $\mathrm{P} 1$ & $\mathrm{~N} 2$ & $106.0(2)$ \\
\hline N1 & $\mathrm{P} 1$ & N3 & 119.1(3) \\
\hline N1 & $\mathrm{P} 1$ & $\mathrm{~N} 2$ & $120.0(2)$ \\
\hline N1 & $\mathrm{P} 1$ & N4 & $112.04(18)$ \\
\hline $\mathrm{C} 7$ & N3 & $\mathrm{P} 1$ & $127.9(2)$ \\
\hline $\mathrm{C} 2$ & N3 & $\mathrm{P} 1$ & $113.9(3)$ \\
\hline $\mathrm{C} 2$ & N3 & $\mathrm{C} 7$ & $118.2(3)$ \\
\hline C3 & $\mathrm{N} 2$ & $\mathrm{P} 1$ & $124.5(4)$ \\
\hline $\mathrm{C} 1$ & $\mathrm{~N} 2$ & $\mathrm{P} 1$ & $109.1(3)$ \\
\hline $\mathrm{C} 1$ & $\mathrm{~N} 2$ & C3 & $116.1(4)$ \\
\hline $\mathrm{C} 11$ & N4 & $\mathrm{P} 1$ & $122.6(3)$ \\
\hline $\mathrm{C} 11$ & N4 & $\mathrm{C} 13$ & $116.9(3)$ \\
\hline $\mathrm{C} 13$ & N4 & $\mathrm{P} 1$ & $120.4(3)$ \\
\hline N4 & $\mathrm{C} 11$ & $\mathrm{C} 12$ & $113.3(3)$ \\
\hline $\mathrm{N} 2$ & $\mathrm{C} 3$ & $\mathrm{C} 4$ & $110.3(4)$ \\
\hline $\mathrm{N} 2$ & C3 & $\mathrm{C} 5$ & $111.7(4)$ \\
\hline $\mathrm{N} 2$ & C3 & C6 & $108.1(4)$ \\
\hline $\mathrm{C} 4$ & $\mathrm{C} 3$ & $\mathrm{C} 5$ & $108.4(4)$ \\
\hline C6 & C3 & $\mathrm{C} 4$ & $108.3(4)$ \\
\hline C6 & C3 & $\mathrm{C} 5$ & $109.9(4)$ \\
\hline N3 & $\mathrm{C} 7$ & $\mathrm{C} 8$ & $108.8(3)$ \\
\hline N3 & $\mathrm{C} 7$ & C9 & $109.7(3)$ \\
\hline N3 & $\mathrm{C} 7$ & $\mathrm{C} 10$ & $111.5(3)$ \\
\hline $\mathrm{C} 9$ & $\mathrm{C} 7$ & $\mathrm{C} 8$ & $110.8(4)$ \\
\hline $\mathrm{C} 10$ & $\mathrm{C} 7$ & $\mathrm{C} 8$ & $108.1(3)$ \\
\hline $\mathrm{C} 10$ & $\mathrm{C} 7$ & C9 & $108.0(4)$ \\
\hline N2 & $\mathrm{C} 1$ & $\mathrm{C} 2$ & $104.1(4)$ \\
\hline N4 & $\mathrm{C} 13$ & $\mathrm{C} 14$ & $114.9(4)$ \\
\hline P1 & N1 & $\mathrm{Tb} 1$ & $164.7(4)$ \\
\hline N3 & $\mathrm{C} 2$ & $\mathrm{C} 1$ & $105.8(3)$ \\
\hline C1S_1 & C2S_1 & C3S_1 & $113.6(4)$ \\
\hline $\mathrm{C}_{4 \mathrm{~S}_{-}}{ }^{-1}$ & $\mathrm{C} 3 \mathrm{~S}^{-} 1$ & $\mathrm{C}_{2} \mathrm{~S}^{-} 1$ & $113.9(4)$ \\
\hline C5S_1 & C4S_1 & C3S_1 & $113.7(4)$ \\
\hline
\end{tabular}




\section{Electronic Absorption Spectra}

General Considerations. Special measures were taken to obtain the UV-visible/NIR spectroscopy of $\mathbf{4}$ as the compound is very air and water sensitive. Spectra were taken immediately after solution preparation from dilution of stock solution with a $240 \mathrm{~nm} / \mathrm{min}$ scan rate.
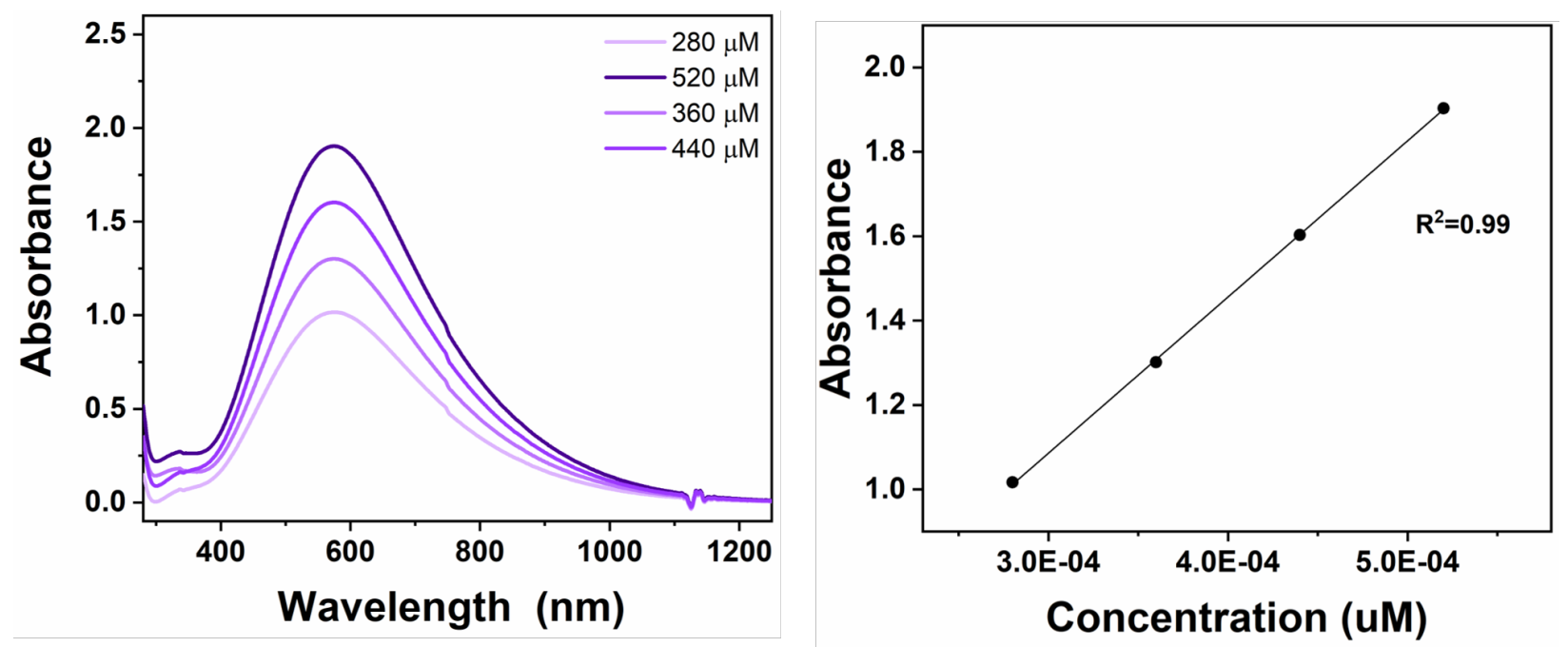

Figure S28. (left) UV-vis/NIR spectra of 4 in benzene. (right) Linear regression of absorbance at $575 \mathrm{~nm}$ maximum where $\varepsilon=3700 \mathrm{~cm}^{-1} \mathrm{M}^{-1}$.

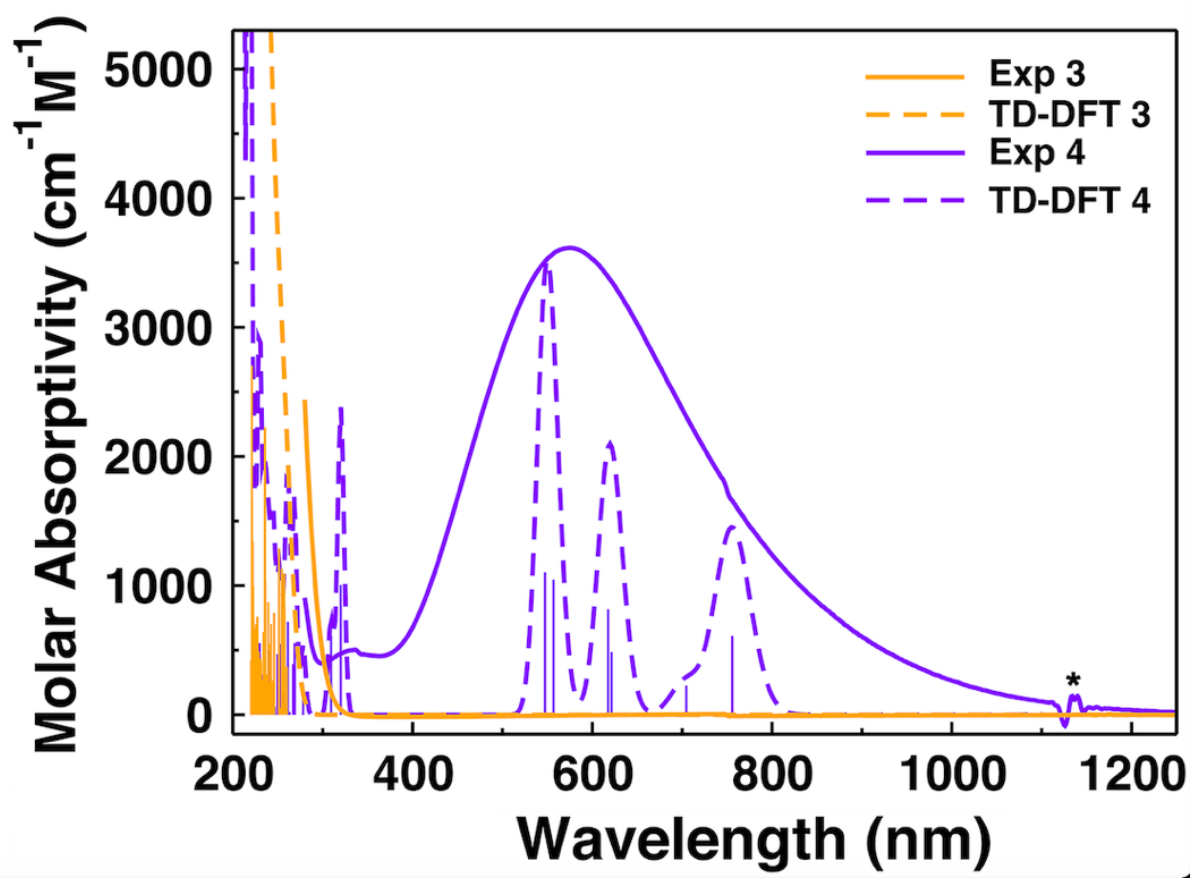

Figure S29. Experimental UV/vis/NIR spectra of $\mathbf{3}$ and $\mathbf{4}$ in benzene (solid lines) and their computed TD-DFT spectra in the UV/vis region (dashed lines). Vertical bars depict theoretical 
oscillator strength of single-electron excitations. A * has been placed on the graphic to denote a grating change for the UV/vis/NIR instrument.

\section{Magnetism}

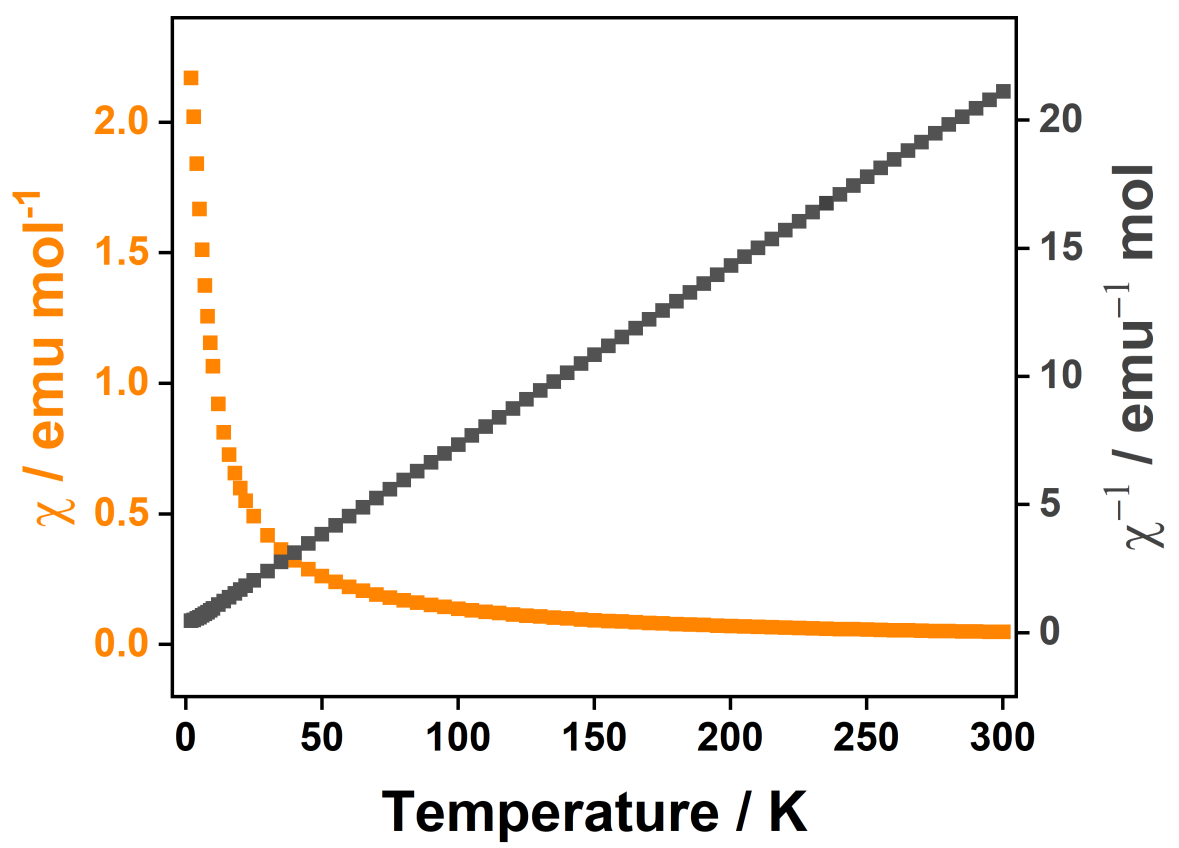

Figure S30. $\chi T$ vs. $T$ (orange, left axis) and $1 / \chi$ vs. $T$ (gray, right axis) for 3.

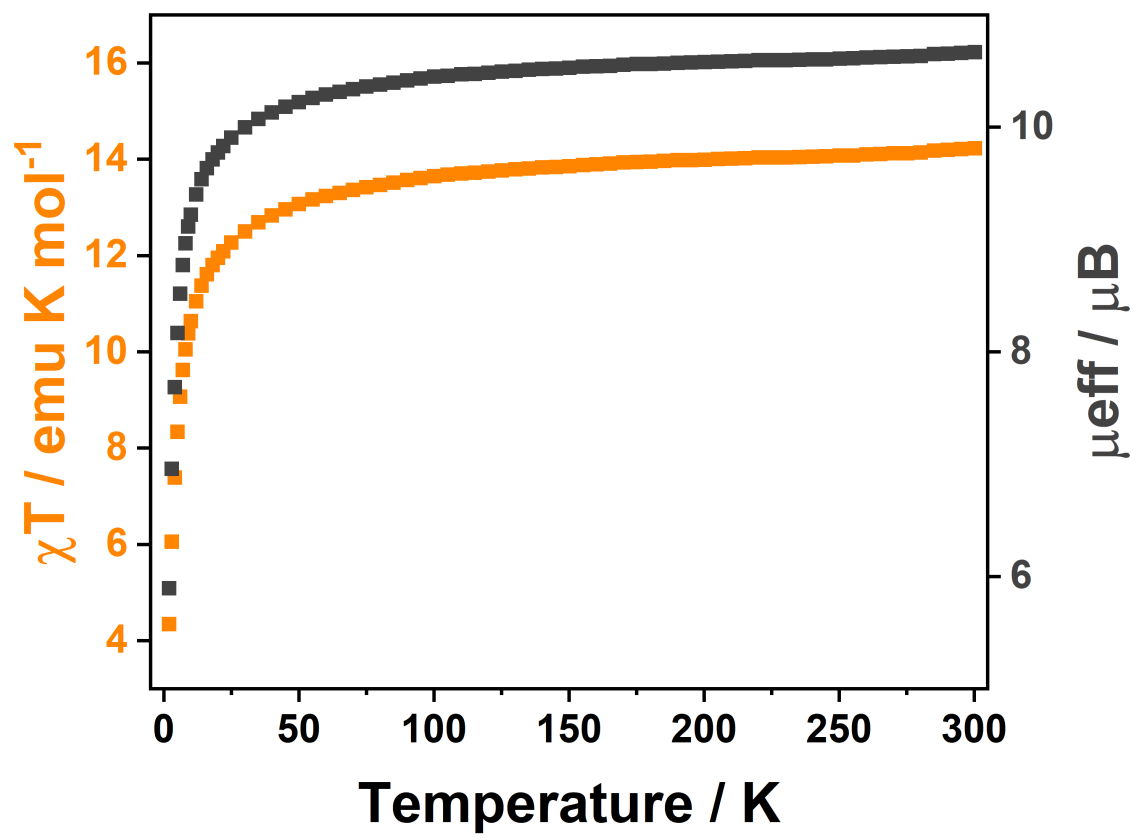

Figure S31. $\chi T$ vs. $T$ (orange, left axis) and $\mu_{\text {eff }}$ vs. $T$ (gray, right axis) plot for 3. 


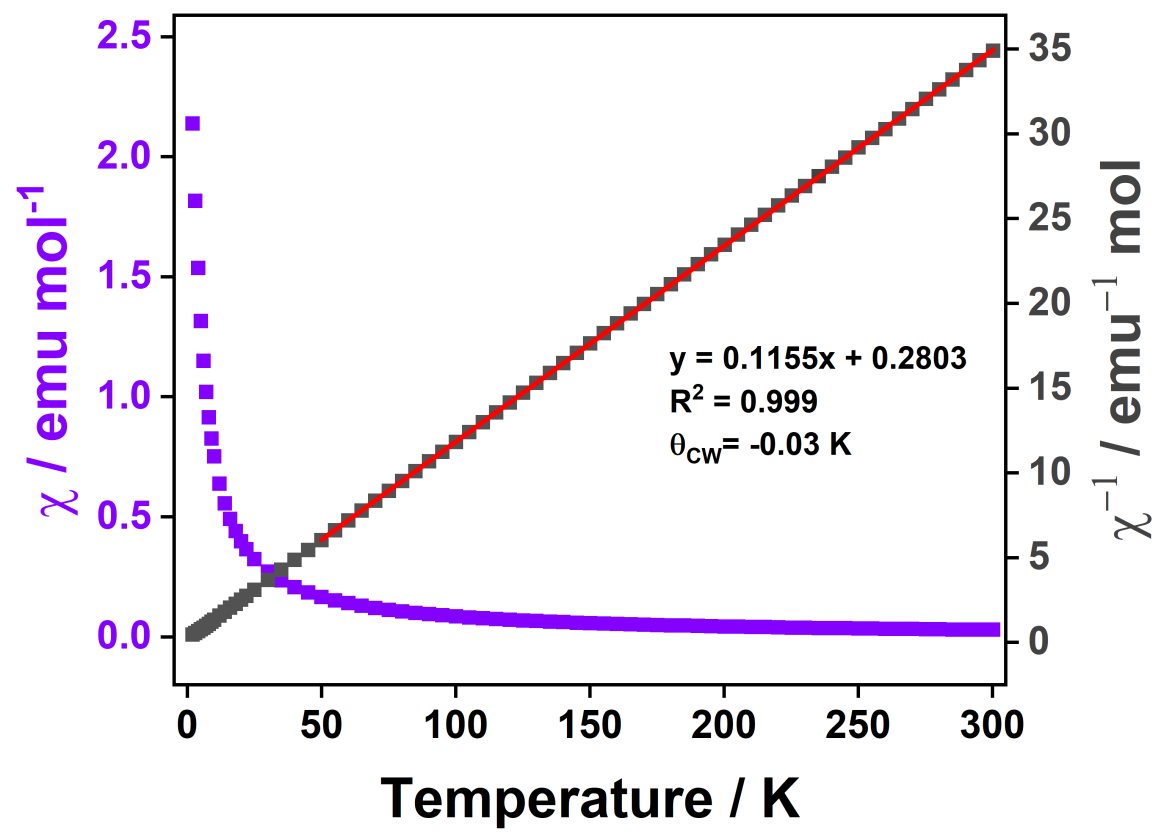

Figure S32. $\chi T$ vs. $T$ (purple, left axis) and $1 / \chi$ vs. $T$ (gray, right axis) for 4.

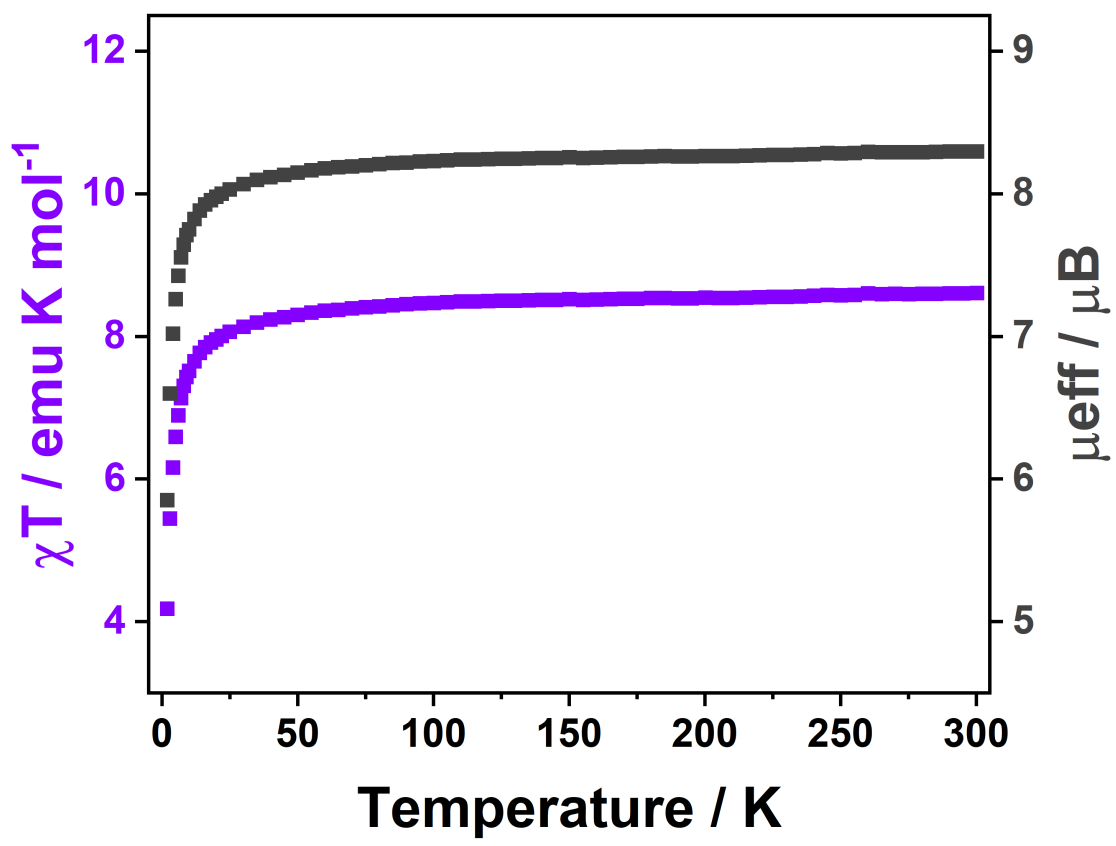

Figure S33. $\chi T$ vs. $T$ (purple, left axis) and $\mu_{\text {eff }}$ vs. $T$ (gray, right axis) plot for 4.

Fitting of Field-Dependent Magnetic Data for 4. A $S=7 / 2$ spin Hamiltonian with only $D$ and isotropic $g$ was employed yielding a satisfactory fit using a previously described approach (the results are consistent with those obtained with PHI). ${ }^{1,2}$ The fit uses all three field datasets, but because at the lower field the high $T$ is less reliable (since $\chi$ is small), only the two higher field (1 $\mathrm{T}, 3 \mathrm{~T})$ were employed for the final fit. The $g$ value is determined from the high $T$ data $(T \geq 100$ 
$\mathrm{K}$ ), but even using the whole $T$ range, the $g$ value changes little, as does $D$. These different constraints are the basis for the error in $D$ of $\pm 0.5 \mathrm{~cm}^{-1}$.

The Hamiltonian used is the sum of the electronic Zeeman (EZ), with only an isotropic $g$ value, and zero-field splitting (ZFS) interactions, with only second order terms.

$\hat{\mathcal{H}}=\hat{\mathcal{H}}_{\mathrm{EZ}}+\hat{\mathcal{H}}_{\mathrm{ZFS}}$

$=g \mu_{\mathrm{B}} \boldsymbol{B} \cdot \hat{\boldsymbol{S}}+\hat{\boldsymbol{S}} \cdot \boldsymbol{D} \cdot \hat{\boldsymbol{S}}$

$=g \mu_{\mathrm{B}} \boldsymbol{B} \cdot \hat{\boldsymbol{S}}+D\left\{\hat{S}_{z}^{2}-\frac{1}{3} S(S+1) \hat{1}\right\}+E\left(\hat{S}_{x}^{2}-\hat{S}_{y}^{2}\right)$

$\mu_{\mathrm{B}}$ is the Bohr magneton, $\hat{1}$ is the identity matrix, and $D$ and $E$ are the axial and rhombic zfs terms, respectively.

Goodness of fit calculated using established procedure. ${ }^{3}$

$1 \mathrm{~T}, 3 \mathrm{~T}, \mathrm{D}=0, g$ varying, but $T>=100 \mathrm{~K}$ only GINSBERG STANDARD ERROR OF ESTIMATE $=0.50274031 \mathrm{E}-01(1 \mathrm{e}-02$ is essentially perfect for any real system)

$1 \mathrm{~T}, 3 \mathrm{~T}, \mathrm{D}>0, g$ varying, all $T$

GINSBERG STANDARD ERROR OF ESTIMATE $=0.13810961 \mathrm{E}+00$

$1 \mathrm{~T}, 3 \mathrm{~T}, \mathrm{D}<0, g$ varying, all $T$

GINSBERG STANDARD ERROR OF ESTIMATE $=0.15132256 \mathrm{E}+00$

$1 \mathrm{~T}, 3 \mathrm{~T}, \mathrm{D}>0, g$ fixed at high $T$ value, all $T$ GINSBERG STANDARD ERROR OF ESTIMATE $=0.14273531 \mathrm{E}+00$ 


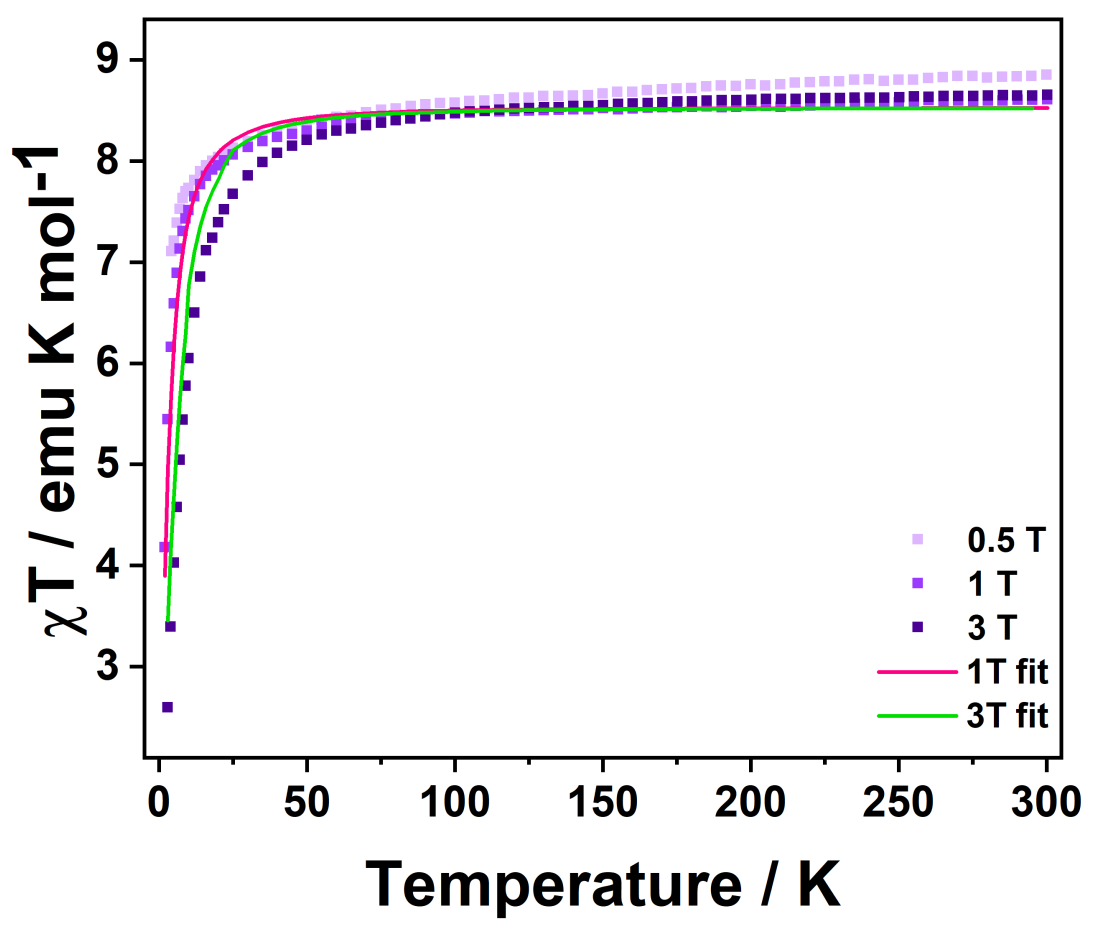

Figure S34. $\chi T$ vs. $T$ plot at $0.5,1$, and $3 \mathrm{~T}$ for 4 and fitting of the 1 and $3 \mathrm{~T}$ data with $\mathrm{S}=7 / 2, D$ $= \pm 5.5 \mathrm{~cm}^{-1}, g=2.085$.

\section{Electron Paramagnetic Resonance}

EPR simulations used the program SPIN (A. Ozarowski, NHMFL). Calculated resonant fields $( \pm 0.5 \mathrm{mT})$ for $v=9.366 \mathrm{GHz}, S=7 / 2, D= \pm 5.5 \mathrm{~cm}^{-1}, E / D=0.11, g=2.085$. The $D$ and $g$ values are based solely on magnetic susceptibility fits, shown in Figure S34.

\begin{tabular}{|l|l|l|l|}
\hline & Orientation & $x$ & $y$ \\
\hline $\begin{array}{l}\left|S=7 / 2, M_{S}\right\rangle \\
\text { multiplet }\end{array}$ & $z$ & 245 & 52 \\
\hline $\pm 1 / 2$ & 542.5 & 131.5 & 133 \\
\hline $\pm 3 / 2$ & 126.5 & --- & --- \\
\hline $\pm 5 / 2$ & 65 & --- & --- \\
\hline $\pm 7 / 2$ & 46 & & \\
\hline
\end{tabular}




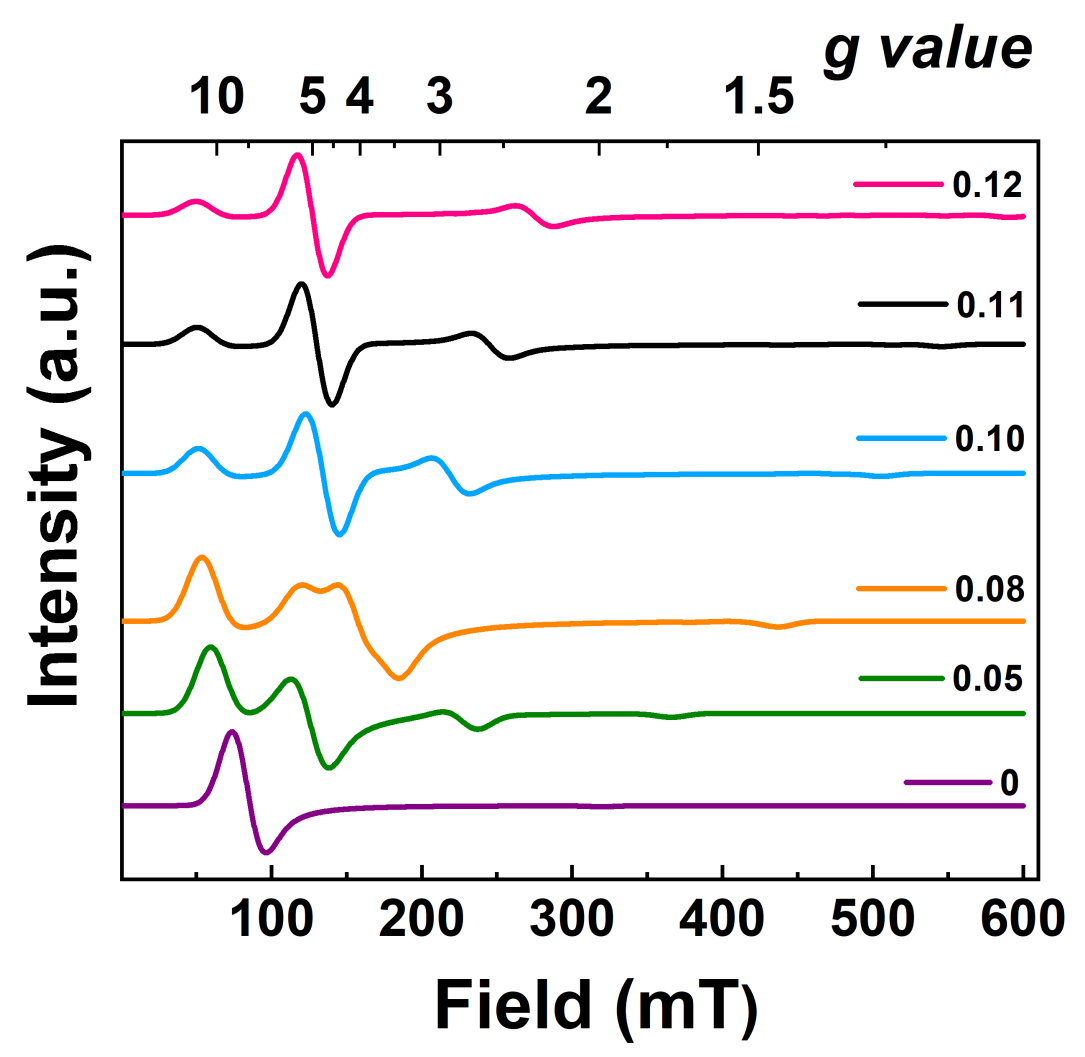

Figure S35. Simulation of EPR spectrum of 4 at $9.366 \mathrm{GHz}$ and $12 \mathrm{~K}$ with changing $|E / D|$ value. The best match between the simulated and experimental features obtains for $|E / D|=$ 0.11 , see main text for comparison with experimental spectrum.

\section{Theoretical Details}

Table S13. Experimental (exp) vs. optimized (opt) Tb-N and N-P bond lengths $(\AA)$ and Tb-N-P valence angles $\left({ }^{\circ}\right)$ of complexes 3 and $\mathbf{4}$. See Figure 1 of the main text for atom labels.

$\begin{array}{ccccc} & \mathbf{3}(\exp ) & \mathbf{3}(\mathrm{opt}) & \mathbf{4}(\exp ) & \mathbf{4} \text { (opt) } \\ \text { Tb1-N1a } & 2.231 & 2.248 & 2.106 & 2.188 \\ \text { Tb1-N1b } & 2.264 & 2.249 & & \\ \text { N1a-P1a } & 1.528 & 1.533 & 1.555 & 1.554 \\ \text { N1b-P1b } & 1.532 & 1.534 & & \\ \text { Tb1-N1a-P1a } & 168.74 & 172.16 & 164.70 & 162.40 \\ \text { Tb1-N1b-P1b } & 167.64 & 173.03 & & \end{array}$




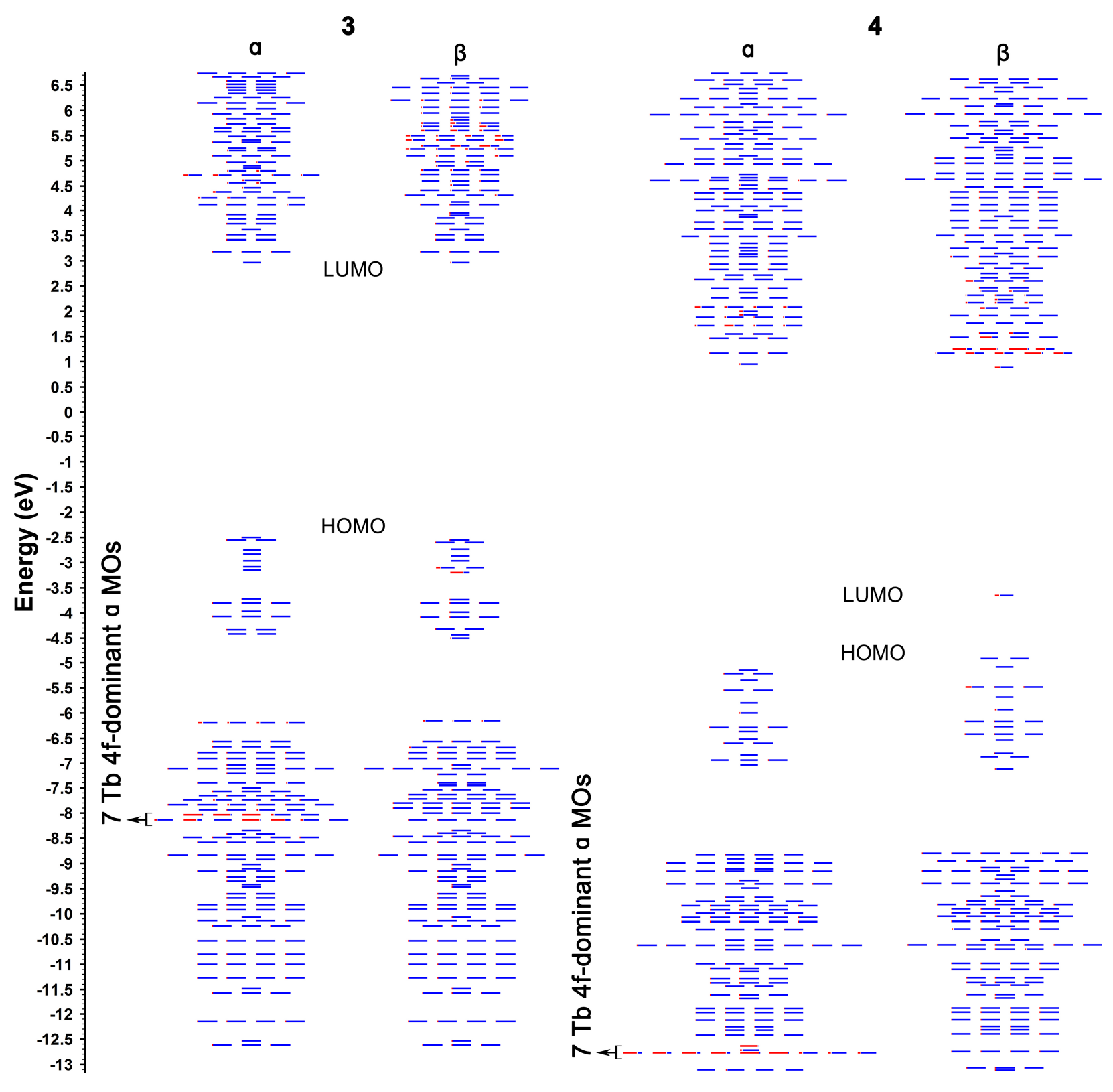

Figure S36. Combined $(\alpha+\beta)$ MO diagrams of 3 and 4. The red fraction of the MO lines represents the percentage of Tb AOs in the MOs, and the blue lines are the ligand fraction. Degeneracy of the $\mathrm{MO}$ energy levels is set to $0.05 \mathrm{eV}$. 


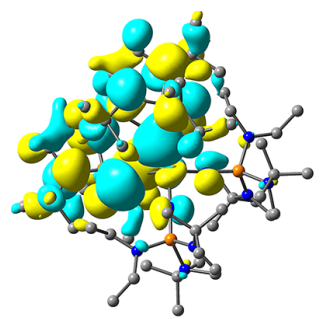

HOMO

$97 \%$ L, $3 \% \mathrm{~Tb}(5 \mathrm{~d})$

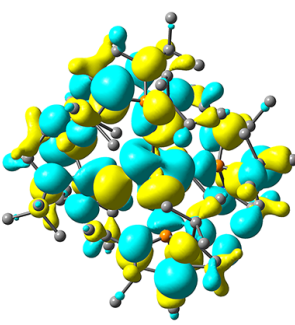

HOMO-5

$65 \% \mathrm{~L}, 35 \% \mathrm{~Tb}(6 \mathrm{~s}, 4 \mathrm{f})$

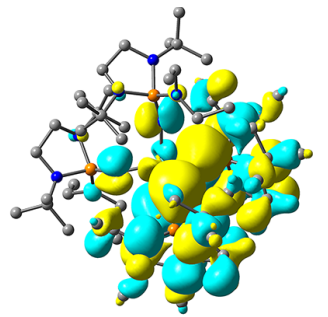

HOMO-1

97\%L, 3\% Tb (5d)

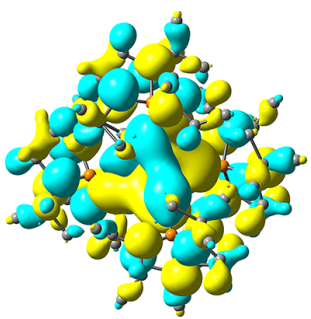

HOMO-2

96\%L, 4\%Tb (5d)

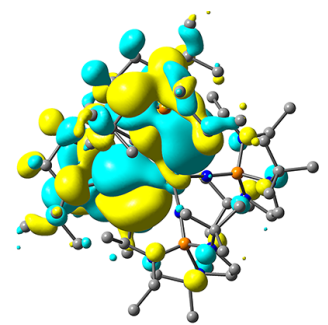

HOMO-3

$98 \%$ L, $2 \% \operatorname{Tb}(5 d)$

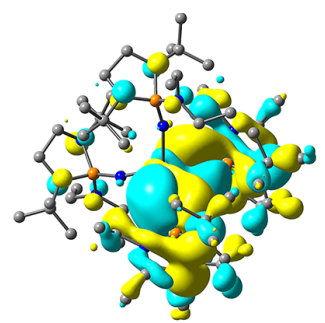

HOMO-4

$98 \%$ L, $2 \%$ Tb (5d)

Figure S37. Ten highest occupied MOs of 4 ( $\beta$ electron density). Fragment composition (\%) of the MOs is shown in colors in Figure 3 of the main text (Tb AOs, red vs. ligands AOs, blue). Dominant AOs of $\mathrm{Tb}$ are shown in parentheses.

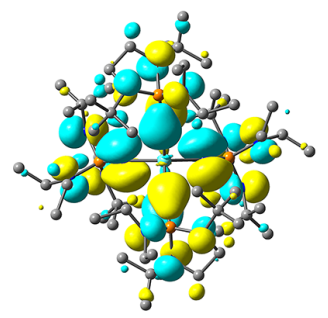

HOMO

$99 \%$ L, $1 \% \mathrm{~Tb}(5 \mathrm{~d})$

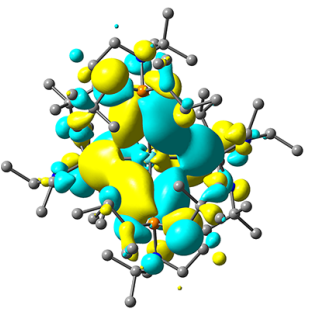

HOMO-5

$97 \% \mathrm{~L}, 3 \% \mathrm{~Tb}(5 \mathrm{~d})$

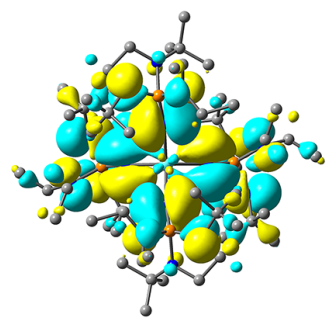

HOMO-1

$99 \%$ L, $1 \%$ Tb $(5 d, 4 f)$

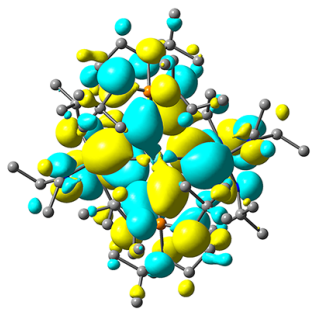

HOMO-2

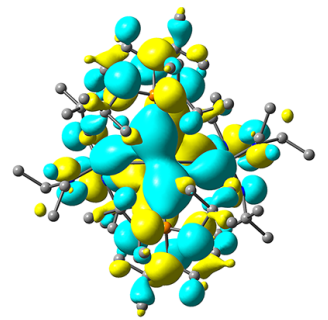

HOMO-3

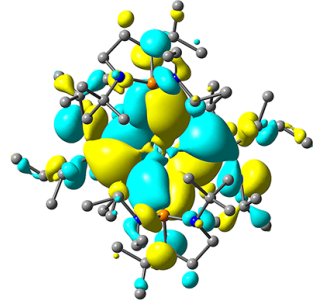

HOMO-4 $98 \% \mathrm{~L}, 2 \% \mathrm{~Tb}(5 \mathrm{~d})$

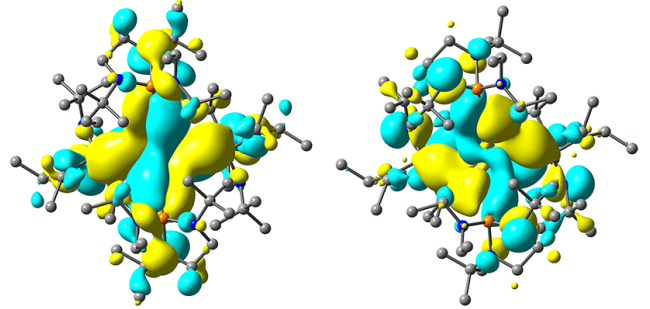

HOMO-6

$96 \%$ L, 4\% Tb (5d)

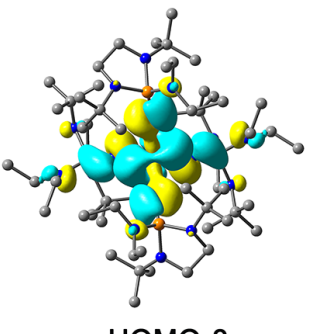

HOMO-8

$75 \%$ L, $25 \% \mathrm{~Tb}(6 \mathrm{~s}, 4 \mathrm{f}) \quad 34 \% \mathrm{~L}, 66 \% \mathrm{~Tb}(6 \mathrm{~s}, 4 \mathrm{f})$

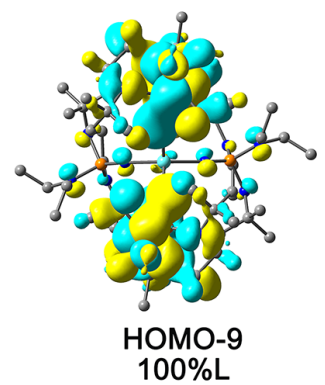

Figure S38. Ten highest occupied MOs of 3 ( $\beta$ electron density). Fragment composition (\%) of the MOs is shown in colors in Figure 3 of the main text (Tb AOs, red vs. ligands AOs, blue). Dominant AOs of $\mathrm{Tb}$ are shown in parentheses. 


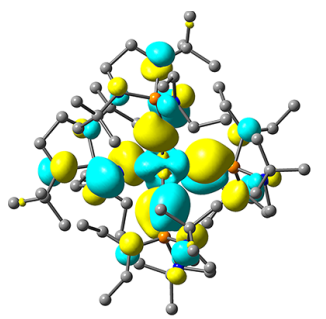

LUMO

$73 \%$ L, $27 \% \operatorname{Tb}(6 s, 4 f)$

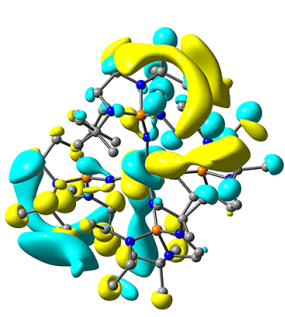

LUMO+5

$54 \% \mathrm{~L}, 46 \% \mathrm{~Tb}(4 \mathrm{f})$

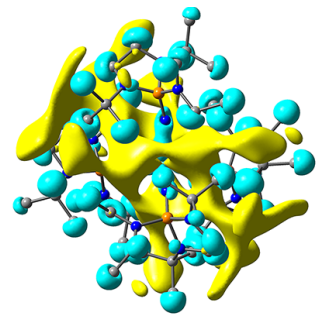

LUMO+1

$68 \% \mathrm{~L}, 32 \% \mathrm{~Tb}(6 s, 4 f)$

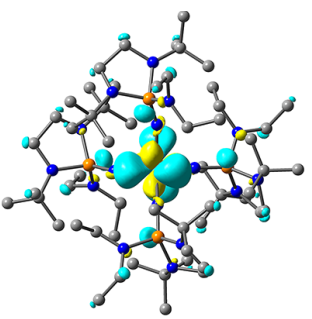

LUMO+2

28\%L, 72\% Tb (4f)

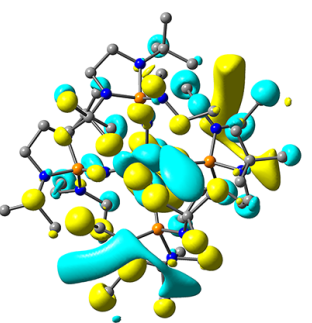

LUMO+7

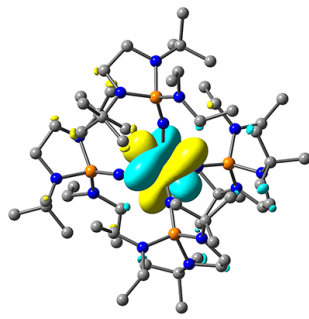

LUMO+6

9\%L, $91 \%$ Tb (4f)

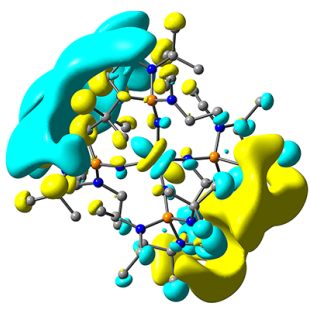

LUMO+3

93\%L, 7\%Tb (4f)

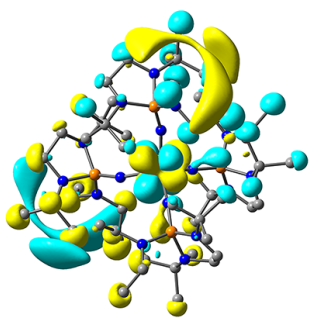

LUMO+8

$46 \%$ L, 54\% Tb (4f)

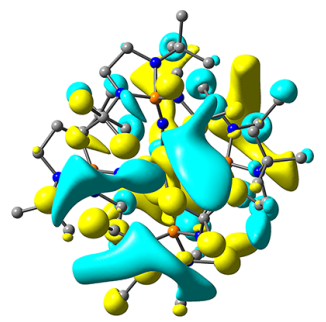

LUMO+4

$54 \% \mathrm{~L}, 46 \% \mathrm{~Tb}(4 \mathrm{f})$

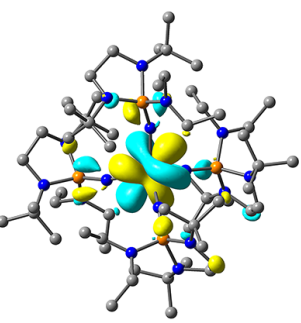

LUMO+9

$8 \%$ L, $92 \%$ Tb (4f)

Figure S39. Ten lowest unoccupied MOs of 4 ( $\beta$ electron density). Fragment composition (\%) of the MOs is shown in colors in Figure 3 of the main text (Tb AOs, red vs. ligands AOs, blue). Dominant AOs of $\mathrm{Tb}$ are shown in parentheses.

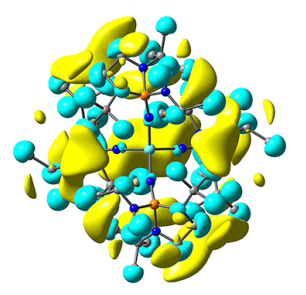

LUMO

$93 \% \mathrm{~L}, 7 \% \mathrm{~Tb}(6 \mathrm{~s})$

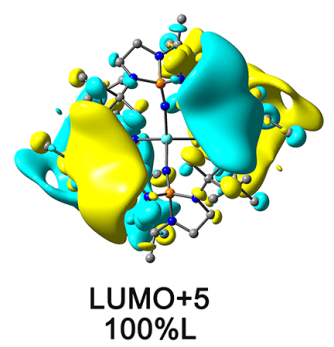

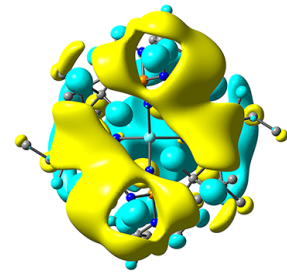

LUMO+1 $100 \% \mathrm{~L}$

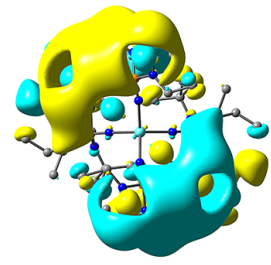

LUMO+6

$99 \% \mathrm{~L}, 1 \% \mathrm{~Tb}(6 \mathrm{p})$

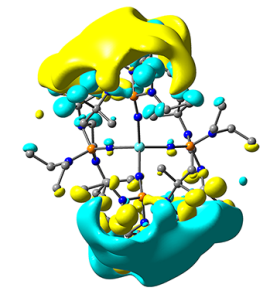

LUMO+2

$100 \% \mathrm{~L}$

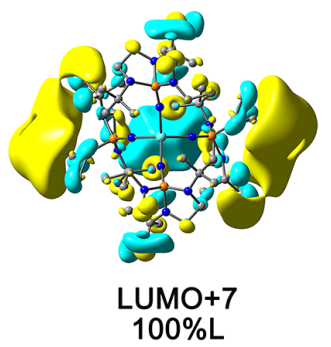

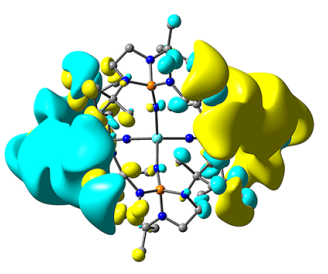

LUMO+3

$100 \% \mathrm{~L}$

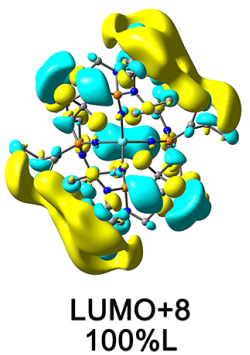

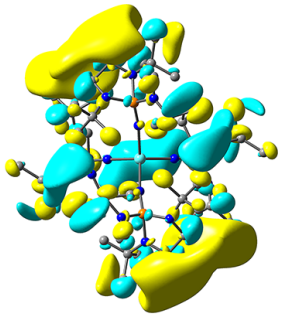

LUMO+4 $100 \% \mathrm{~L}$

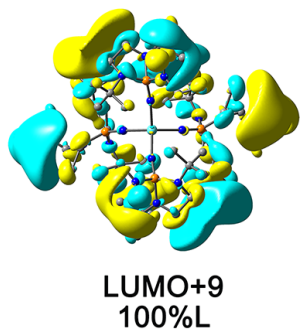

Figure S40. Ten lowest unoccupied MOs of 3 ( $\beta$ electron density). Fragment composition (\%) of the MOs is shown in colors in Figure 3 of the main text (Tb AOs, red vs. ligands AOs, blue). Dominant $\mathrm{AOs}$ of $\mathrm{Tb}$ are shown in parentheses. 
Table S14. Bond polarization (\%) of the NBOs shown in Figure 4 of the main text.

\begin{tabular}{c|c|c|c|c|c|c|c}
\hline \multirow{2}{*}{ Bonds } & \multicolumn{2}{|c|}{ Tb-N $\boldsymbol{\sigma}$ bond } & \multicolumn{2}{c|}{$\mathbf{N}-\mathbf{P} \boldsymbol{\sigma}$ bond } & \multicolumn{3}{c}{ Tb-N-P $\boldsymbol{\pi}$ bonds } \\
\cline { 2 - 8 } & $\mathbf{T b}$ & $\mathbf{N}$ & $\mathbf{N}$ & $\mathbf{P}$ & $\mathbf{T b}$ & $\mathbf{N}$ & $\mathbf{P}$ \\
$\mathbf{3}$ & 5.23 & 94.77 & 70.00 & 30.00 & 2.24 & 95.57 & 2.19 \\
$\mathbf{4}$ & 8.31 & 91.69 & 70.42 & 29.58 & 5.69 & 92.58 & 1.74
\end{tabular}

Table S15. Tb hybrids (\%) of the Tb NBOs.

\begin{tabular}{|c|c|c|c|c|c|c|c|c|}
\hline \multirow[t]{2}{*}{ Bonds } & \multicolumn{4}{|c|}{3} & \multicolumn{4}{|c|}{4} \\
\hline & $\mathbf{S}$ & $\mathbf{p}$ & d & f & $\mathbf{S}$ & $\mathbf{p}$ & d & f \\
\hline $\mathrm{Tb}-\mathrm{N} \sigma$ bond & 15.27 & 0.40 & 73.49 & 10.62 & 18.13 & 0.34 & 75.19 & 6.10 \\
\hline $\mathrm{Tb}-\mathrm{N}-\mathrm{P} \pi$ bonds & 2.83 & 0.99 & 72.12 & 32.23 & 9.71 & 2.11 & 62.11 & 25.44 \\
\hline $1 \mathrm{c}-1 \mathrm{e} \alpha \mathrm{NBO}$ on $\mathrm{Tb}$ & 0.00 & 0.01 & 0.01 & 99.98 & 0.00 & 0.01 & 0.01 & 99.98 \\
\hline $1 \mathrm{c}-1 \mathrm{e} \beta \mathrm{NBO}$ on $\mathrm{Tb}$ & 57.59 & 0.00 & 0.01 & 42.40 & \multicolumn{4}{|c|}{$\mathrm{N} / \mathrm{A}$} \\
\hline
\end{tabular}

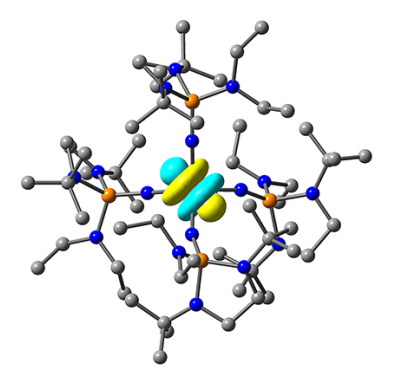

$1 c-1 e a$
$O N=1.00|e|$

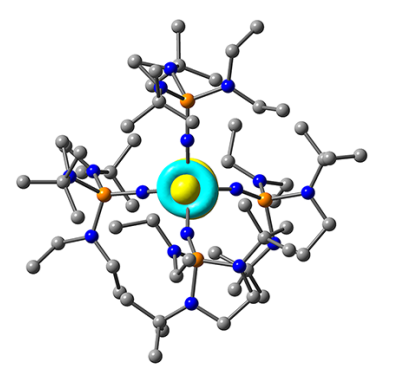

$1 \mathrm{c}-1 \mathrm{e}$ a $\mathrm{ON}=1.00|\mathrm{e}|$

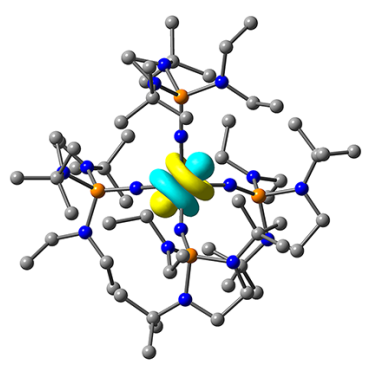

$1 \mathrm{c}-1 \mathrm{e}$ a $\mathrm{ON}=1.00|\mathrm{e}|$

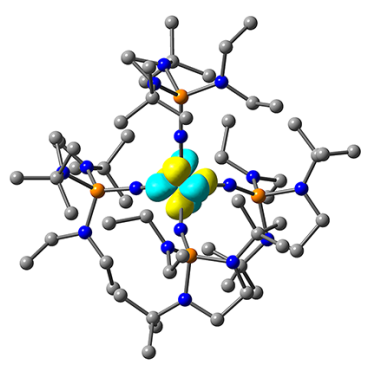

$1 \mathrm{c}-1 \mathrm{e}$ a $\mathrm{ON}=1.00|\mathrm{e}|$

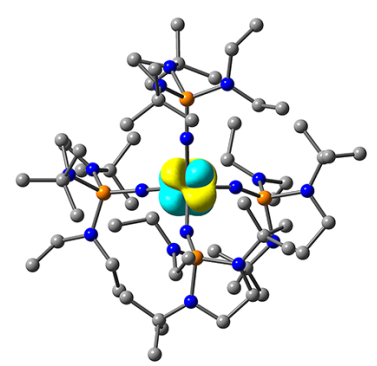

$1 \mathrm{c}-1 \mathrm{e}$ a $\mathrm{ON}=1.00|\mathrm{e}|$

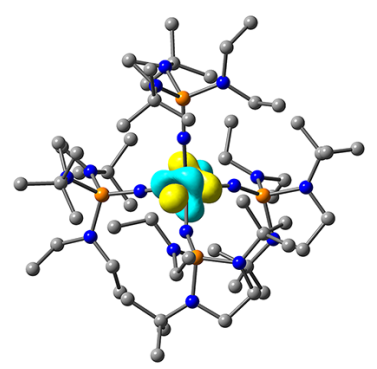

$1 \mathrm{c}-1 \mathrm{e} a$ $\mathrm{ON}=1.00|\mathrm{e}|$

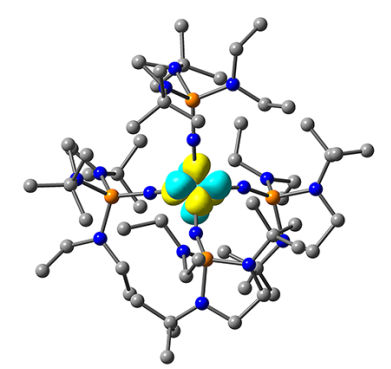

$1 \mathrm{c}-1 \mathrm{e}$ a $\mathrm{ON}=1.00|\mathrm{e}|$

Figure S41. Seven unpaired electrons (1c-1e $\alpha$ NBOs) on Tb in 4. 
A
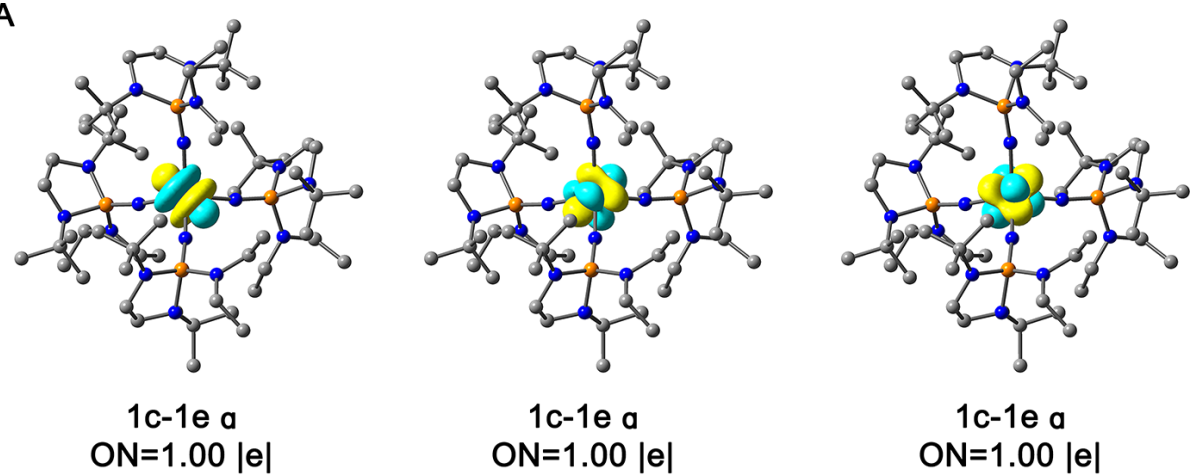

1c-1e a

$\mathrm{ON}=1.00|\mathrm{e}|$
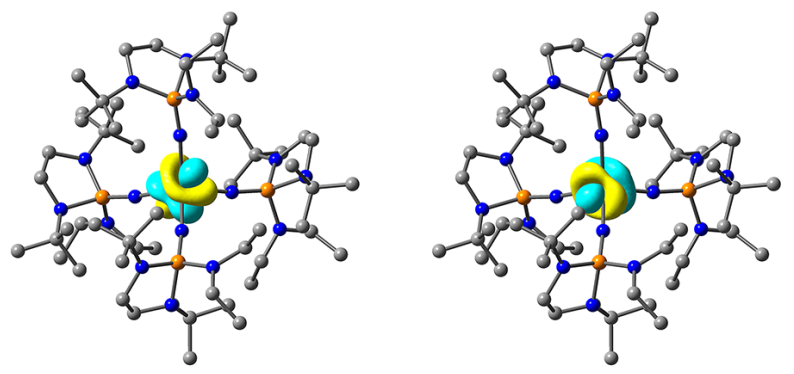

$1 \mathrm{c}-1 \mathrm{e} \mathrm{a}$ $\mathrm{ON}=1.00|\mathrm{e}|$
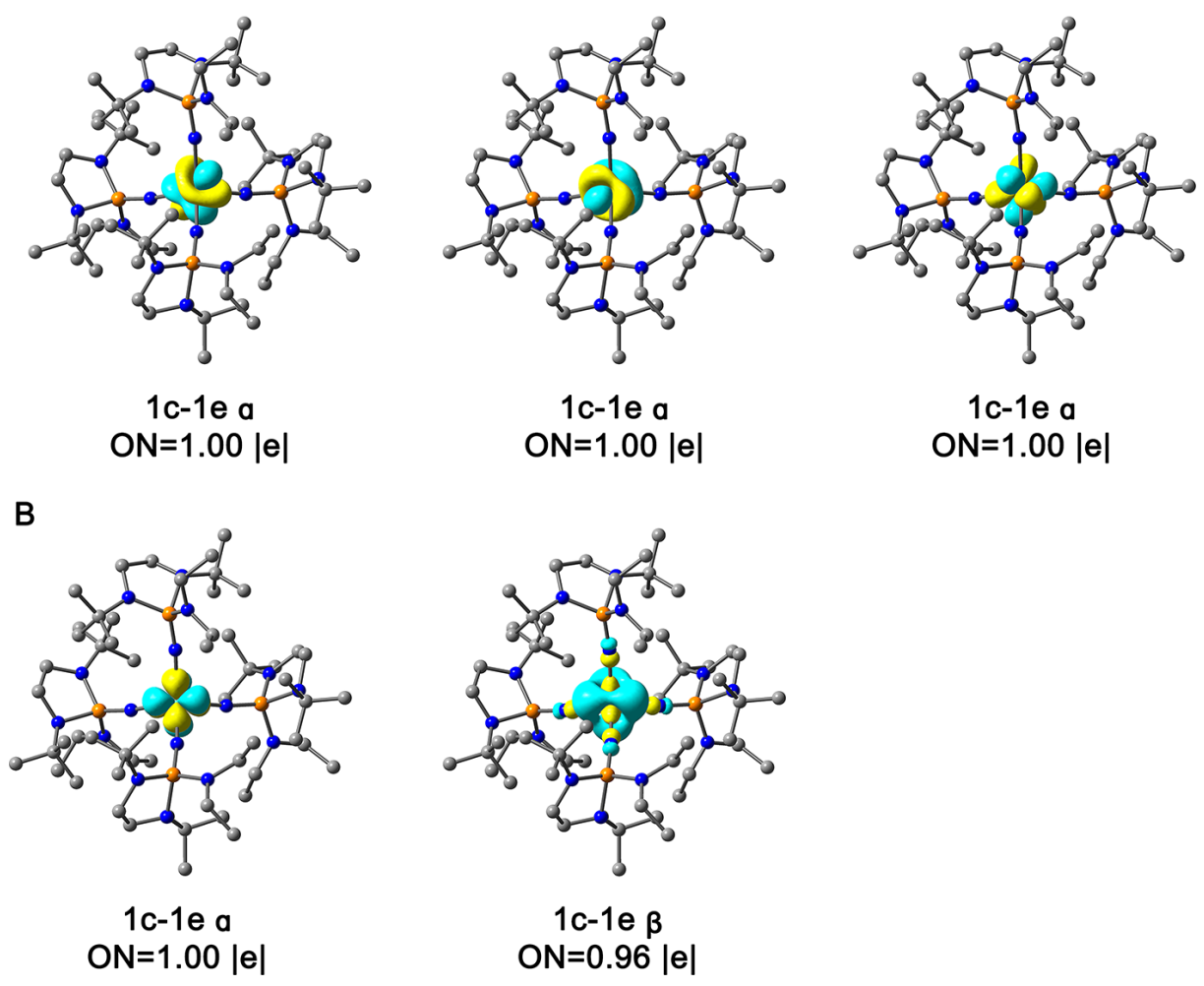

Figure S42. (A) Six unpaired electrons (1c-1e $\alpha$ NBOs) and (B) two electrons $(\alpha+\beta)$ composing a lone pair (1c-2e NBO) on $\mathrm{Tb}$ in 3. 
A

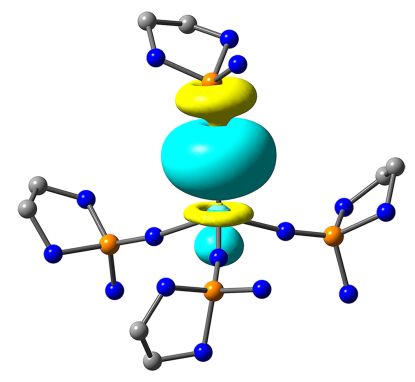

2c-2e Tb-N $\sigma$ bond $\mathrm{ON}=1.96|\mathrm{e}|$

C

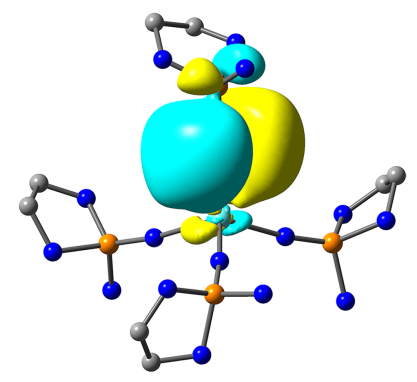

$3 c-2 e$ Tb-N-P $\pi$ bond $\mathrm{ON}=1.93|\mathrm{e}|$
B

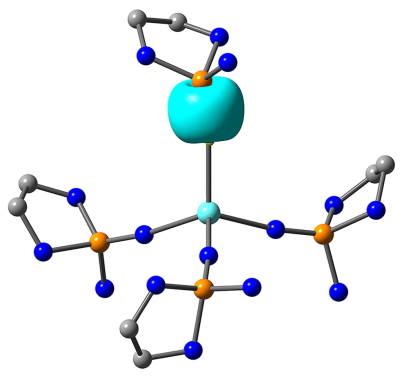

2c-2e P-N $\sigma$ bond $\mathrm{ON}=1.98|\mathrm{e}|$

D

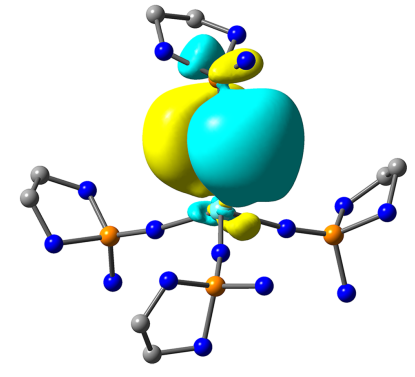

3c-2e Tb-N-P m bond $\mathrm{ON}=1.94|\mathrm{e}|$

Figure S43. Bonding analysis of the $\mathrm{Tb}-\mathrm{N}-\mathrm{P}$ interactions in 3. (A) Two-center two-electron $\mathrm{Tb}-$ $\mathrm{N} \sigma$ bond. (B) Two-center two-electron $\mathrm{P}-\mathrm{N} \sigma$ bond. (C, D) Three-center two-electron Tb-N-P $\pi$ bonds. ON denotes occupation number here and elsewhere. Side groups of the ligands $\left({ }^{t} \mathrm{Bu}_{\mathrm{B}} \mathrm{Et}_{2}\right)$ are omitted for simplicity. An equivalent set of bonds is identified for other three ligands. 
A

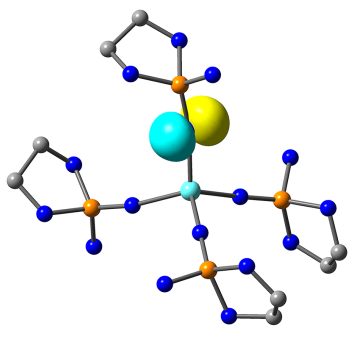

1c-2e p-type LP on N

$\mathrm{ON}=1.58|\mathrm{e}|$

B

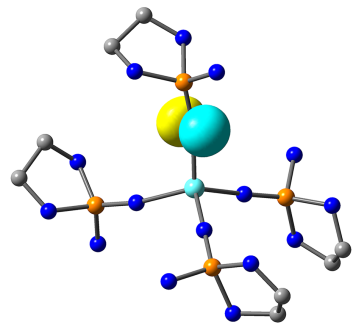

1c-2e p-type LP on $\mathrm{N}$ $\mathrm{ON}=1.71|\mathrm{e}|$

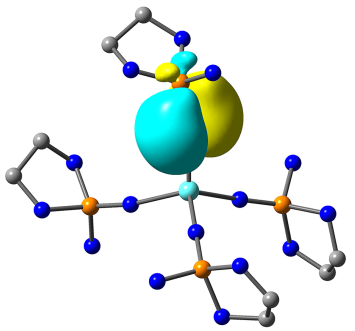

2c-2e N-P $\pi$ bond $\mathrm{ON}=1.76|\mathrm{e}|$

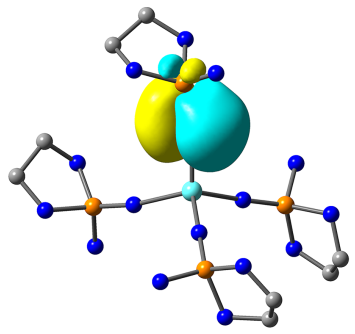

2c-2e N-P $\pi$ bond $\mathrm{ON}=1.87|\mathrm{e}|$

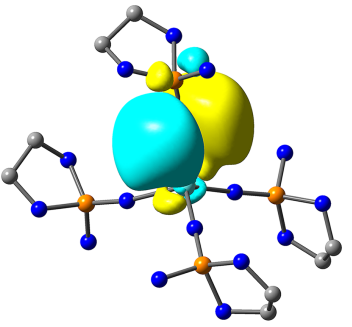

$3 c-2 e$ Tb-N-P $\pi$ bond $\mathrm{ON}=1.94|\mathrm{e}|$

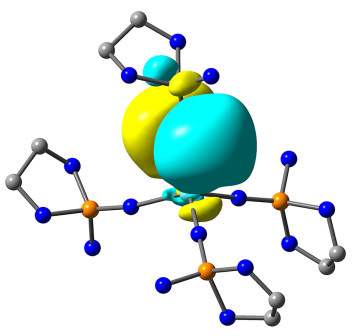

$3 c-2 e$ Tb-N-P $\pi$ bond $\mathrm{ON}=1.95|\mathrm{e}|$

Figure S44. Representations of (A) two orthogonal p-type lone pairs of $N$ as (B) two two-center two-electron $\mathrm{N}-\mathrm{P} \pi$ bonds and $(\mathrm{C})$ two three-center two-electron $\mathrm{Tb}-\mathrm{N}-\mathrm{P} \pi$ bonds in 4 . Side groups of the ligands $\left({ }^{t} \mathrm{Bu}, \mathrm{Et}_{2}\right)$ are omitted for simplicity.

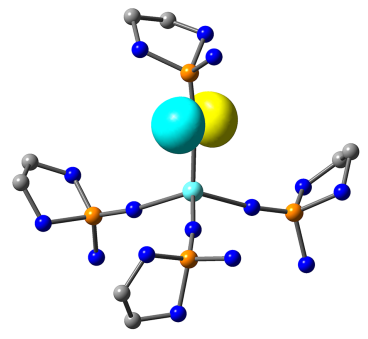

1c-2e p-type LP on N $\mathrm{ON}=1.66|\mathrm{e}|$

B

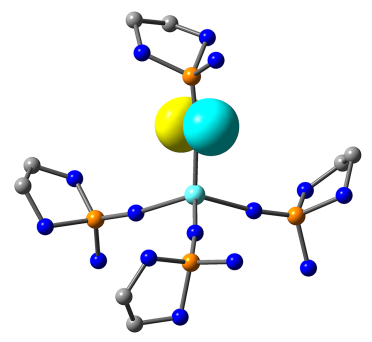

1c-2e p-type LP on N $\mathrm{ON}=1.69|\mathrm{e}|$

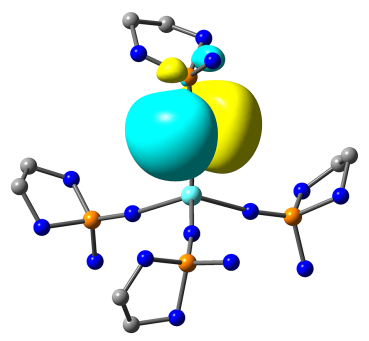

2c-2e N-P $\pi$ bond $\mathrm{ON}=1.88|\mathrm{e}|$

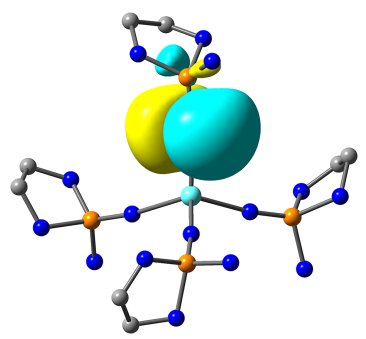

2c-2e N-P $\pi$ bond $\mathrm{ON}=1.89|\mathrm{e}|$

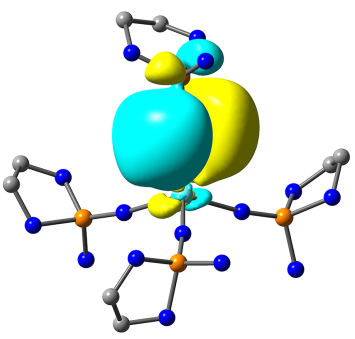

$3 \mathrm{c}-2 \mathrm{e}$ Tb-N-P $\pi$ bond $\mathrm{ON}=1.93|\mathrm{e}|$

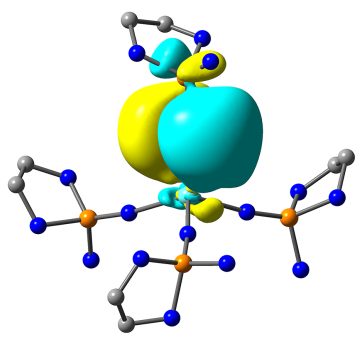

$3 \mathrm{c}-2 \mathrm{e}$ Tb-N-P $\pi$ bond $\mathrm{ON}=1.94|\mathrm{e}|$

Figure S45. Representations of (A) two orthogonal p-type lone pairs of $\mathrm{N}$ as (B) two two-center two-electron $\mathrm{N}-\mathrm{P} \pi$ bonds and $(\mathrm{C})$ two three-center two-electron $\mathrm{Tb}-\mathrm{N}-\mathrm{P} \pi$ bonds in $\mathbf{3}$. Side groups of the ligands $\left({ }^{t} \mathrm{Bu}, \mathrm{Et}_{2}\right)$ are omitted for simplicity. 
Table S16. Cartesian coordinates of optimized complexes 3 and 4.

\begin{tabular}{|c|c|c|c|c|c|c|c|}
\hline \multicolumn{4}{|c|}{3} & \multicolumn{4}{|c|}{4} \\
\hline $\mathrm{Tb}$ & 0.00000000 & 0.00000000 & 0.02806200 & $\mathrm{~Tb}$ & 0.00000000 & 0.00000000 & 0.00000000 \\
\hline $\mathrm{P}$ & -1.47314000 & 2.73846500 & 2.16523000 & $\mathrm{P}$ & 1.03065400 & 2.75097500 & 2.24771600 \\
\hline $\mathrm{N}$ & -0.76173300 & 1.66581600 & 1.33170000 & $\mathrm{~N}$ & 2.40763300 & 2.76500100 & 3.25005800 \\
\hline $\mathrm{N}$ & -0.49328200 & 3.40085400 & 3.39519700 & $\mathrm{~N}$ & 0.00000000 & 2.90592400 & 3.61553600 \\
\hline $\mathrm{N}$ & -2.97747000 & 2.40071700 & 2.98495600 & $\mathrm{~N}$ & 0.99893200 & 4.26924000 & 1.51197900 \\
\hline $\mathrm{N}$ & -2.17947000 & 4.12667700 & 1.43765900 & $\mathrm{C}$ & 0.64529900 & 4.43793300 & 0.11542000 \\
\hline $\mathrm{C}$ & -4.06560200 & 2.91992900 & 2.17829300 & $\mathrm{H}$ & -0.35067900 & 4.89717500 & 0.02722900 \\
\hline $\mathrm{H}$ & -4.98402900 & 3.01787000 & 2.76568700 & $\mathrm{H}$ & 0.55905400 & 3.43493300 & -0.30652800 \\
\hline $\mathrm{H}$ & -4.28452400 & 2.28494800 & 1.30631400 & $\mathrm{C}$ & -1.47276900 & 2.82596900 & 3.56252200 \\
\hline $\mathrm{C}$ & -3.58746000 & 4.27965900 & 1.70966000 & $\mathrm{C}$ & -1.98858400 & 3.28881900 & 2.20287700 \\
\hline $\mathrm{H}$ & -4.14304200 & 4.59135000 & 0.81647300 & $\mathrm{H}$ & -1.67486900 & 4.30946900 & 1.98055300 \\
\hline $\mathrm{H}$ & -3.77660900 & 5.03440000 & 2.49393900 & $\mathrm{H}$ & -3.08101500 & 3.26384100 & 2.20788300 \\
\hline $\mathrm{C}$ & -1.51643600 & 5.12562200 & 0.59263600 & $\mathrm{H}$ & -1.64354100 & 2.64157300 & 1.39351700 \\
\hline $\mathrm{C}$ & -1.87107900 & 6.53854900 & 1.07476300 & $\mathrm{C}$ & 3.81823800 & 2.71122000 & 2.83908000 \\
\hline $\mathrm{H}$ & -2.94121400 & 6.74863700 & 0.98322000 & $\mathrm{C}$ & 0.64943300 & 2.30109600 & 4.76401800 \\
\hline $\mathrm{H}$ & -1.33879500 & 7.28374200 & 0.47507600 & $\mathrm{H}$ & 0.58022700 & 1.20300200 & 4.75184600 \\
\hline $\mathrm{H}$ & -1.58405200 & 6.67703800 & 2.12093900 & $\mathrm{H}$ & 0.20446200 & 2.65557900 & 5.69785000 \\
\hline $\mathrm{C}$ & -1.96737800 & 4.96557800 & -0.86305500 & $\mathrm{C}$ & -1.96887800 & 1.40106800 & 3.82000400 \\
\hline $\mathrm{H}$ & -1.69209400 & 3.97815000 & -1.23480500 & $\mathrm{H}$ & -1.53486700 & 0.69084600 & 3.11053900 \\
\hline $\mathrm{H}$ & -1.49827500 & 5.72289500 & -1.50056900 & $\mathrm{H}$ & -3.05700500 & 1.36061500 & 3.72279700 \\
\hline $\mathrm{H}$ & -3.05089700 & 5.07658700 & -0.96609000 & $\mathrm{H}$ & -1.72536400 & 1.06583400 & 4.83315000 \\
\hline $\mathrm{C}$ & 0.00000000 & 4.96708300 & 0.65396800 & $\mathrm{C}$ & 1.64331600 & 5.26093600 & -0.68730600 \\
\hline $\mathrm{H}$ & 0.38114100 & 5.21043900 & 1.64702200 & $\mathrm{H}$ & 1.72299200 & 6.29129000 & -0.32753700 \\
\hline $\mathrm{H}$ & 0.46268900 & 5.64437300 & -0.06989200 & $\mathrm{H}$ & 1.32497400 & 5.31008600 & -1.73243700 \\
\hline $\mathrm{H}$ & 0.30204300 & 3.94439800 & 0.41806500 & $\mathrm{H}$ & 2.64080500 & 4.81772100 & -0.66036100 \\
\hline $\mathrm{C}$ & -3.21074100 & 1.10464700 & 3.65047300 & $\mathrm{C}$ & -2.04594100 & 3.76294800 & 4.63175100 \\
\hline $\mathrm{C}$ & -3.60798700 & 0.00670100 & 2.65818600 & $\mathrm{H}$ & -1.75342000 & 3.46607800 & 5.64285200 \\
\hline $\mathrm{H}$ & -4.57331200 & 0.21502300 & 2.18789700 & $\mathrm{H}$ & -3.13959000 & 3.75367500 & 4.59708900 \\
\hline $\mathrm{H}$ & -3.69601600 & -0.95950100 & 3.16546200 & $\mathrm{H}$ & -1.70485800 & 4.78828200 & 4.46826800 \\
\hline $\mathrm{H}$ & -2.85563000 & -0.07987200 & 1.87126500 & $\mathrm{C}$ & 0.21001800 & 6.51956000 & 2.28975300 \\
\hline $\mathrm{C}$ & -4.32518700 & 1.28052400 & 4.68809200 & $\mathrm{H}$ & -0.70645200 & 6.13726200 & 2.74455000 \\
\hline $\mathrm{H}$ & -4.07784800 & 2.08662700 & 5.38505800 & $\mathrm{H}$ & -0.03452200 & 6.87303100 & 1.28414900 \\
\hline $\mathrm{H}$ & -4.45341100 & 0.35612000 & 5.26020500 & $\mathrm{H}$ & 0.53509800 & 7.38841100 & 2.87019900 \\
\hline $\mathrm{H}$ & -5.29058300 & 1.51049700 & 4.22803500 & $\mathrm{C}$ & 4.36521300 & 1.29631000 & 3.04279200 \\
\hline $\mathrm{C}$ & -1.94767300 & 0.66960100 & 4.38960900 & $\mathrm{H}$ & 4.28674600 & 0.97834000 & 4.08660000 \\
\hline $\mathrm{H}$ & -1.12810500 & 0.48870900 & 3.69215300 & $\mathrm{H}$ & 5.42167500 & 1.24110400 & 2.76057100 \\
\hline $\mathrm{H}$ & -2.14320400 & -0.26039300 & 4.93068000 & $\mathrm{H}$ & 3.79939100 & 0.59418100 & 2.42960500 \\
\hline $\mathrm{H}$ & -1.62944800 & 1.42430300 & 5.11096600 & $\mathrm{C}$ & 4.63185600 & 3.70464400 & 3.67549100 \\
\hline $\mathrm{C}$ & -1.16475300 & 4.20343400 & 5.67860700 & $\mathrm{H}$ & 4.23000300 & 4.71704100 & 3.57667900 \\
\hline $\mathrm{H}$ & -1.45692400 & 5.11254600 & 6.21582500 & $\mathrm{H}$ & 5.67036500 & 3.71676900 & 3.33243600 \\
\hline $\mathrm{H}$ & -0.25931000 & 3.81709300 & 6.15672100 & $\mathrm{H}$ & 4.64274200 & 3.44270400 & 4.73711000 \\
\hline $\mathrm{H}$ & -1.95864700 & 3.46469300 & 5.80715000 & $\mathrm{C}$ & 1.30515100 & 5.46248600 & 2.28150300 \\
\hline $\mathrm{C}$ & -0.94924500 & 4.51129400 & 4.20160500 & $\mathrm{H}$ & 2.23775400 & 5.91374100 & 1.90950300 \\
\hline $\mathrm{H}$ & -1.88844200 & 4.86727800 & 3.77565500 & $\mathrm{H}$ & 1.50842700 & 5.15313800 & 3.30793400 \\
\hline $\mathrm{H}$ & -0.24105800 & 5.35127900 & 4.11184100 & $\mathrm{C}$ & 3.96743800 & 3.09073800 & 1.36976500 \\
\hline $\mathrm{C}$ & 0.75963600 & 2.77447100 & 3.75510000 & $\mathrm{H}$ & 3.34130400 & 2.46399600 & 0.73184200 \\
\hline $\mathrm{H}$ & 0.96187700 & 2.03340200 & 2.97921300 & $\mathrm{H}$ & 5.00946700 & 2.95792900 & 1.06494100 \\
\hline $\mathrm{H}$ & 0.66488600 & 2.21874300 & 4.70226400 & $\mathrm{H}$ & 3.69591000 & 4.13441400 & 1.20447000 \\
\hline $\mathrm{C}$ & 1.93126600 & 3.74058700 & 3.86554500 & $\mathrm{~N}$ & 0.86796700 & 1.58295300 & 1.23556400 \\
\hline $\mathrm{H}$ & 2.07711500 & 4.29037400 & 2.93268800 & $\mathrm{C}$ & 2.10074800 & 2.72877500 & 4.66423900 \\
\hline $\mathrm{H}$ & 2.85182900 & 3.18685400 & 4.07604800 & $\mathrm{H}$ & 2.23758300 & 3.71893700 & 5.12702500 \\
\hline $\mathrm{H}$ & 1.80191500 & 4.47058700 & 4.67183000 & $\mathrm{H}$ & 2.74461200 & 2.02215400 & 5.19939100 \\
\hline $\mathrm{P}$ & 2.73749500 & 1.28132400 & -2.23582600 & $\mathrm{P}$ & -1.03065400 & -2.75097500 & 2.24771600 \\
\hline $\mathrm{N}$ & 1.69980700 & 0.75925900 & -1.23380900 & $\mathrm{~N}$ & -2.40763300 & -2.76500100 & 3.25005800 \\
\hline $\mathrm{N}$ & 3.70249300 & 2.54857000 & -1.62744400 & $\mathrm{~N}$ & 0.00000000 & -2.90592400 & 3.61553600 \\
\hline $\mathrm{N}$ & 2.27041800 & 1.86821700 & -3.77929000 & $\mathrm{~N}$ & -0.99893200 & -4.26924000 & 1.51197900 \\
\hline $\mathrm{N}$ & 3.90096600 & 0.22039300 & -2.99069300 & $\mathrm{C}$ & -0.64529900 & -4.43793300 & 0.11542000 \\
\hline $\mathrm{C}$ & 2.82389500 & 1.12928300 & -4.88857700 & $\mathrm{H}$ & 0.35067900 & -4.89717500 & 0.02722900 \\
\hline
\end{tabular}




\begin{tabular}{|c|c|c|c|c|c|c|c|}
\hline $\mathrm{H}$ & 3.60825900 & 1.70779900 & -5.40691900 & $\mathrm{H}$ & -0.55905400 & -3.43493300 & -0.30652800 \\
\hline $\mathrm{H}$ & 2.05908200 & 0.87858600 & -5.63415300 & $\mathrm{C}$ & 1.47276900 & -2.82596900 & 3.56252200 \\
\hline $\mathrm{C}$ & 3.41043000 & -0.14523100 & -4.30591600 & $\mathrm{C}$ & 1.98858400 & -3.28881900 & 2.20287700 \\
\hline $\mathrm{H}$ & 2.63461300 & -0.92362700 & -4.26608600 & $\mathrm{H}$ & 1.67486900 & -4.30946900 & 1.98055300 \\
\hline $\mathrm{H}$ & 4.22202100 & -0.51395300 & -4.94116300 & $\mathrm{H}$ & 3.08101500 & -3.26384100 & 2.20788300 \\
\hline $\mathrm{C}$ & 4.57434100 & -0.84349000 & -2.22331600 & $\mathrm{H}$ & 1.64354100 & -2.64157300 & 1.39351700 \\
\hline $\mathrm{C}$ & 5.83266700 & -1.27736700 & -2.98312200 & $\mathrm{C}$ & -3.81823800 & -2.71122000 & 2.83908000 \\
\hline $\mathrm{H}$ & 5.59733600 & -1.78738700 & -3.92175100 & $\mathrm{C}$ & -0.64943300 & -2.30109600 & 4.76401800 \\
\hline $\mathrm{H}$ & 6.41465500 & -1.97648700 & -2.37432100 & $\mathrm{H}$ & -0.58022700 & -1.20300200 & 4.75184600 \\
\hline $\mathrm{H}$ & 6.46205800 & -0.41215500 & -3.21211700 & $\mathrm{H}$ & -0.20446200 & -2.65557900 & 5.69785000 \\
\hline $\mathrm{C}$ & 3.67148900 & -2.05755500 & -1.98935300 & $\mathrm{C}$ & 1.96887800 & -1.40106800 & 3.82000400 \\
\hline $\mathrm{H}$ & 2.78146400 & -1.75945300 & -1.43131600 & $\mathrm{H}$ & 1.53486700 & -0.69084600 & 3.11053900 \\
\hline $\mathrm{H}$ & 4.20129900 & -2.82101000 & -1.41083300 & $\mathrm{H}$ & 3.05700500 & -1.36061500 & 3.72279700 \\
\hline $\mathrm{H}$ & 3.35733500 & -2.52245000 & -2.92899500 & $\mathrm{H}$ & 1.72536400 & -1.06583400 & 4.83315000 \\
\hline $\mathrm{C}$ & 5.00473100 & -0.30073600 & -0.86295300 & $\mathrm{C}$ & -1.64331600 & -5.26093600 & -0.68730600 \\
\hline $\mathrm{H}$ & 5.65213900 & 0.57154900 & -0.96451600 & $\mathrm{H}$ & -1.72299200 & -6.29129000 & -0.32753700 \\
\hline $\mathrm{H}$ & 5.55530700 & -1.07523800 & -0.32007900 & $\mathrm{H}$ & -1.32497400 & -5.31008600 & -1.73243700 \\
\hline $\mathrm{H}$ & 4.13457300 & -0.02322000 & -0.26576900 & $\mathrm{H}$ & -2.64080500 & -4.81772100 & -0.66036100 \\
\hline $\mathrm{C}$ & 1.17734600 & 2.81158000 & -4.03285900 & $\mathrm{C}$ & 2.04594100 & -3.76294800 & 4.63175100 \\
\hline $\mathrm{C}$ & -0.13345000 & 2.05619000 & -4.27332700 & $\mathrm{H}$ & 1.75342000 & -3.46607800 & 5.64285200 \\
\hline $\mathrm{H}$ & -0.05270100 & 1.38263900 & -5.13322900 & $\mathrm{H}$ & 3.13959000 & -3.75367500 & 4.59708900 \\
\hline $\mathrm{H}$ & -0.95637000 & 2.75161600 & -4.47413600 & $\mathrm{H}$ & 1.70485800 & -4.78828200 & 4.46826800 \\
\hline $\mathrm{H}$ & -0.38608000 & 1.45464800 & -3.39687900 & $\mathrm{C}$ & -0.21001800 & -6.51956000 & 2.28975300 \\
\hline $\mathrm{C}$ & 1.51796200 & 3.66621400 & -5.25907000 & $\mathrm{H}$ & 0.70645200 & -6.13726200 & 2.74455000 \\
\hline $\mathrm{H}$ & 2.46697900 & 4.19117500 & -5.11273800 & $\mathrm{H}$ & 0.03452200 & -6.87303100 & 1.28414900 \\
\hline $\mathrm{H}$ & 0.73583800 & 4.41460800 & -5.42008500 & $\mathrm{H}$ & -0.53509800 & -7.38841100 & 2.87019900 \\
\hline $\mathrm{H}$ & 1.59449300 & 3.07106500 & -6.17374700 & $\mathrm{C}$ & -4.36521300 & -1.29631000 & 3.04279200 \\
\hline $\mathrm{C}$ & 0.99851400 & 3.75184700 & -2.84431600 & $\mathrm{H}$ & -4.28674600 & -0.97834000 & 4.08660000 \\
\hline $\mathrm{H}$ & 0.77949700 & 3.19527600 & -1.93143600 & $\mathrm{H}$ & -5.42167500 & -1.24110400 & 2.76057100 \\
\hline $\mathrm{H}$ & 0.16476000 & 4.43168600 & -3.04075400 & $\mathrm{H}$ & -3.79939100 & -0.59418100 & 2.42960500 \\
\hline $\mathrm{H}$ & 1.89934400 & 4.34600400 & -2.67507000 & $\mathrm{C}$ & -4.63185600 & -3.70464400 & 3.67549100 \\
\hline $\mathrm{C}$ & 6.09808800 & 3.04970700 & -2.20611700 & $\mathrm{H}$ & -4.23000300 & -4.71704100 & 3.57667900 \\
\hline $\mathrm{H}$ & 6.35944200 & 3.28153000 & -1.16865500 & $\mathrm{H}$ & -5.67036500 & -3.71676900 & 3.33243600 \\
\hline $\mathrm{H}$ & 6.70585300 & 3.69554700 & -2.84986900 & $\mathrm{H}$ & -4.64274200 & -3.44270400 & 4.73711000 \\
\hline $\mathrm{H}$ & 6.37928800 & 2.01351800 & -2.40478500 & $\mathrm{C}$ & -1.30515100 & -5.46248600 & 2.28150300 \\
\hline $\mathrm{C}$ & 4.61622400 & 3.26929300 & -2.48703900 & $\mathrm{H}$ & -2.23775400 & -5.91374100 & 1.90950300 \\
\hline $\mathrm{H}$ & 4.39835100 & 4.34753200 & -2.43081000 & $\mathrm{H}$ & -1.50842700 & -5.15313800 & 3.30793400 \\
\hline $\mathrm{H}$ & 4.39911800 & 2.97825800 & -3.51604100 & $\mathrm{C}$ & -3.96743800 & -3.09073800 & 1.36976500 \\
\hline $\mathrm{C}$ & 3.73135100 & 2.84336700 & -0.20996900 & $\mathrm{H}$ & -3.34130400 & -2.46399600 & 0.73184200 \\
\hline $\mathrm{H}$ & 4.63401000 & 2.42381000 & 0.26628100 & $\mathrm{H}$ & -5.00946700 & -2.95792900 & 1.06494100 \\
\hline $\mathrm{H}$ & 2.87820900 & 2.32180500 & 0.23006800 & $\mathrm{H}$ & -3.69591000 & -4.13441400 & 1.20447000 \\
\hline $\mathrm{C}$ & 3.65378000 & 4.32858400 & 0.11327000 & $\mathrm{~N}$ & -0.86796700 & -1.58295300 & 1.23556400 \\
\hline $\mathrm{H}$ & 2.76361900 & 4.78284400 & -0.32786700 & $\mathrm{C}$ & -2.10074800 & -2.72877500 & 4.66423900 \\
\hline $\mathrm{H}$ & 4.52974200 & 4.88222700 & -0.24124400 & $\mathrm{H}$ & -2.23758300 & -3.71893700 & 5.12702500 \\
\hline $\mathrm{H}$ & 3.60163700 & 4.46803900 & 1.19675500 & $\mathrm{H}$ & -2.74461200 & -2.02215400 & 5.19939100 \\
\hline $\mathrm{P}$ & -2.73749500 & -1.28132400 & -2.23582600 & $\mathrm{P}$ & -2.75097500 & 1.03065400 & -2.24771600 \\
\hline $\mathrm{N}$ & -1.69980700 & -0.75925900 & -1.23380900 & $\mathrm{~N}$ & -2.76500100 & 2.40763300 & -3.25005800 \\
\hline $\mathrm{N}$ & -3.70249300 & -2.54857000 & -1.62744400 & $\mathrm{~N}$ & -2.90592400 & 0.00000000 & -3.61553600 \\
\hline $\mathrm{N}$ & -2.27041800 & -1.86821700 & -3.77929000 & $\mathrm{~N}$ & -4.26924000 & 0.99893200 & -1.51197900 \\
\hline $\mathrm{N}$ & -3.90096600 & -0.22039300 & -2.99069300 & $\mathrm{C}$ & -4.43793300 & 0.64529900 & -0.11542000 \\
\hline $\mathrm{C}$ & -2.82389500 & -1.12928300 & -4.88857700 & $\mathrm{H}$ & -4.89717500 & -0.35067900 & -0.02722900 \\
\hline $\mathrm{H}$ & -3.60825900 & -1.70779900 & -5.40691900 & $\mathrm{H}$ & -3.43493300 & 0.55905400 & 0.30652800 \\
\hline $\mathrm{H}$ & -2.05908200 & -0.87858600 & -5.63415300 & $\mathrm{C}$ & -2.82596900 & -1.47276900 & -3.56252200 \\
\hline $\mathrm{C}$ & -3.41043000 & 0.14523100 & -4.30591600 & $\mathrm{C}$ & -3.28881900 & -1.98858400 & -2.20287700 \\
\hline $\mathrm{H}$ & -2.63461300 & 0.92362700 & -4.26608600 & $\mathrm{H}$ & -4.30946900 & -1.67486900 & -1.98055300 \\
\hline $\mathrm{H}$ & -4.22202100 & 0.51395300 & -4.94116300 & $\mathrm{H}$ & -3.26384100 & -3.08101500 & -2.20788300 \\
\hline $\mathrm{C}$ & -4.57434100 & 0.84349000 & -2.22331600 & $\mathrm{H}$ & -2.64157300 & -1.64354100 & -1.39351700 \\
\hline $\mathrm{C}$ & -5.83266700 & 1.27736700 & -2.98312200 & $\mathrm{C}$ & -2.71122000 & 3.81823800 & -2.83908000 \\
\hline $\mathrm{H}$ & -5.59733600 & 1.78738700 & -3.92175100 & $\mathrm{C}$ & -2.30109600 & 0.64943300 & -4.76401800 \\
\hline $\mathrm{H}$ & -6.41465500 & 1.97648700 & -2.37432100 & $\mathrm{H}$ & -1.20300200 & 0.58022700 & -4.75184600 \\
\hline $\mathrm{H}$ & -6.46205800 & 0.41215500 & -3.21211700 & $\mathrm{H}$ & -2.65557900 & 0.20446200 & -5.69785000 \\
\hline $\mathrm{C}$ & -3.67148900 & 2.05755500 & -1.98935300 & $\mathrm{C}$ & -1.40106800 & -1.96887800 & -3.82000400 \\
\hline
\end{tabular}




\begin{tabular}{|c|c|c|c|c|c|c|c|}
\hline $\mathrm{H}$ & -2.78146400 & 1.75945300 & -1.43131600 & $\mathrm{H}$ & -0.69084600 & -1.53486700 & -3.11053900 \\
\hline $\mathrm{H}$ & -4.20129900 & 2.82101000 & -1.41083300 & $\mathrm{H}$ & -1.36061500 & -3.05700500 & -3.72279700 \\
\hline $\mathrm{H}$ & -3.35733500 & 2.52245000 & -2.92899500 & $\mathrm{H}$ & -1.06583400 & -1.72536400 & -4.83315000 \\
\hline $\mathrm{C}$ & -5.00473100 & 0.30073600 & -0.86295300 & $\mathrm{C}$ & -5.26093600 & 1.64331600 & 0.68730600 \\
\hline $\mathrm{H}$ & -5.65213900 & -0.57154900 & -0.96451600 & $\mathrm{H}$ & -6.29129000 & 1.72299200 & 0.32753700 \\
\hline $\mathrm{H}$ & -5.55530700 & 1.07523800 & -0.32007900 & $\mathrm{H}$ & -5.31008600 & 1.32497400 & 1.73243700 \\
\hline $\mathrm{H}$ & -4.13457300 & 0.02322000 & -0.26576900 & $\mathrm{H}$ & -4.81772100 & 2.64080500 & 0.66036100 \\
\hline $\mathrm{C}$ & -1.17734600 & -2.81158000 & -4.03285900 & $\mathrm{C}$ & -3.76294800 & -2.04594100 & -4.63175100 \\
\hline $\mathrm{C}$ & 0.13345000 & -2.05619000 & -4.27332700 & $\mathrm{H}$ & -3.46607800 & -1.75342000 & -5.64285200 \\
\hline $\mathrm{H}$ & 0.05270100 & -1.38263900 & -5.13322900 & $\mathrm{H}$ & -3.75367500 & -3.13959000 & -4.59708900 \\
\hline $\mathrm{H}$ & 0.95637000 & -2.75161600 & -4.47413600 & $\mathrm{H}$ & -4.78828200 & -1.70485800 & -4.46826800 \\
\hline $\mathrm{H}$ & 0.38608000 & -1.45464800 & -3.39687900 & $\mathrm{C}$ & -6.51956000 & 0.21001800 & -2.28975300 \\
\hline $\mathrm{C}$ & -1.51796200 & -3.66621400 & -5.25907000 & $\mathrm{H}$ & -6.13726200 & -0.70645200 & -2.74455000 \\
\hline $\mathrm{H}$ & -2.46697900 & -4.19117500 & -5.11273800 & $\mathrm{H}$ & -6.87303100 & -0.03452200 & -1.28414900 \\
\hline $\mathrm{H}$ & -0.73583800 & -4.41460800 & -5.42008500 & $\mathrm{H}$ & -7.38841100 & 0.53509800 & -2.87019900 \\
\hline $\mathrm{H}$ & -1.59449300 & -3.07106500 & -6.17374700 & $\mathrm{C}$ & -1.29631000 & 4.36521300 & -3.04279200 \\
\hline $\mathrm{C}$ & -0.99851400 & -3.75184700 & -2.84431600 & $\mathrm{H}$ & -0.97834000 & 4.28674600 & -4.08660000 \\
\hline $\mathrm{H}$ & -0.77949700 & -3.19527600 & -1.93143600 & $\mathrm{H}$ & -1.24110400 & 5.42167500 & -2.76057100 \\
\hline $\mathrm{H}$ & -0.16476000 & -4.43168600 & -3.04075400 & $\mathrm{H}$ & -0.59418100 & 3.79939100 & -2.42960500 \\
\hline $\mathrm{H}$ & -1.89934400 & -4.34600400 & -2.67507000 & $\mathrm{C}$ & -3.70464400 & 4.63185600 & -3.67549100 \\
\hline $\mathrm{C}$ & -6.09808800 & -3.04970700 & -2.20611700 & $\mathrm{H}$ & -4.71704100 & 4.23000300 & -3.57667900 \\
\hline $\mathrm{H}$ & -6.37928800 & -2.01351800 & -2.40478500 & $\mathrm{H}$ & -3.71676900 & 5.67036500 & -3.33243600 \\
\hline $\mathrm{H}$ & -6.35944200 & -3.28153000 & -1.16865500 & $\mathrm{H}$ & -3.44270400 & 4.64274200 & -4.73711000 \\
\hline $\mathrm{H}$ & -6.70585300 & -3.69554700 & -2.84986900 & $\mathrm{C}$ & -5.46248600 & 1.30515100 & -2.28150300 \\
\hline $\mathrm{C}$ & -4.61622400 & -3.26929300 & -2.48703900 & $\mathrm{H}$ & -5.91374100 & 2.23775400 & -1.90950300 \\
\hline $\mathrm{H}$ & -4.39835100 & -4.34753200 & -2.43081000 & $\mathrm{H}$ & -5.15313800 & 1.50842700 & -3.30793400 \\
\hline $\mathrm{H}$ & -4.39911800 & -2.97825800 & -3.51604100 & $\mathrm{C}$ & -3.09073800 & 3.96743800 & -1.36976500 \\
\hline $\mathrm{C}$ & -3.73135100 & -2.84336700 & -0.20996900 & $\mathrm{H}$ & -2.46399600 & 3.34130400 & -0.73184200 \\
\hline $\mathrm{H}$ & -4.63401000 & -2.42381000 & 0.26628100 & $\mathrm{H}$ & -2.95792900 & 5.00946700 & -1.06494100 \\
\hline $\mathrm{H}$ & -2.87820900 & -2.32180500 & 0.23006800 & $\mathrm{H}$ & -4.13441400 & 3.69591000 & -1.20447000 \\
\hline $\mathrm{C}$ & -3.65378000 & -4.32858400 & 0.11327000 & $\mathrm{~N}$ & -1.58295300 & 0.86796700 & -1.23556400 \\
\hline $\mathrm{H}$ & -4.52974200 & -4.88222700 & -0.24124400 & $\mathrm{C}$ & -2.72877500 & 2.10074800 & -4.66423900 \\
\hline $\mathrm{H}$ & -3.60163700 & -4.46803900 & 1.19675500 & $\mathrm{H}$ & -3.71893700 & 2.23758300 & -5.12702500 \\
\hline $\mathrm{H}$ & -2.76361900 & -4.78284400 & -0.32786700 & $\mathrm{H}$ & -2.02215400 & 2.74461200 & -5.19939100 \\
\hline $\mathrm{P}$ & 1.47314000 & -2.73846500 & 2.16523000 & $\mathrm{P}$ & 2.75097500 & -1.03065400 & -2.24771600 \\
\hline $\mathrm{N}$ & 0.76173300 & -1.66581600 & 1.33170000 & $\mathrm{~N}$ & 2.76500100 & -2.40763300 & -3.25005800 \\
\hline $\mathrm{N}$ & 0.49328200 & -3.40085400 & 3.39519700 & $\mathrm{~N}$ & 2.90592400 & 0.00000000 & -3.61553600 \\
\hline $\mathrm{N}$ & 2.97747000 & -2.40071700 & 2.98495600 & $\mathrm{~N}$ & 4.26924000 & -0.99893200 & -1.51197900 \\
\hline $\mathrm{N}$ & 2.17947000 & -4.12667700 & 1.43765900 & $\mathrm{C}$ & 4.43793300 & -0.64529900 & -0.11542000 \\
\hline $\mathrm{C}$ & 4.06560200 & -2.91992900 & 2.17829300 & $\mathrm{H}$ & 4.89717500 & 0.35067900 & -0.02722900 \\
\hline $\mathrm{H}$ & 4.98402900 & -3.01787000 & 2.76568700 & $\mathrm{H}$ & 3.43493300 & -0.55905400 & 0.30652800 \\
\hline $\mathrm{H}$ & 4.28452400 & -2.28494800 & 1.30631400 & $\mathrm{C}$ & 2.82596900 & 1.47276900 & -3.56252200 \\
\hline $\mathrm{C}$ & 3.58746000 & -4.27965900 & 1.70966000 & $\mathrm{C}$ & 3.28881900 & 1.98858400 & -2.20287700 \\
\hline $\mathrm{H}$ & 4.14304200 & -4.59135000 & 0.81647300 & $\mathrm{H}$ & 4.30946900 & 1.67486900 & -1.98055300 \\
\hline $\mathrm{H}$ & 3.77660900 & -5.03440000 & 2.49393900 & $\mathrm{H}$ & 3.26384100 & 3.08101500 & -2.20788300 \\
\hline $\mathrm{C}$ & 1.51643600 & -5.12562200 & 0.59263600 & $\mathrm{H}$ & 2.64157300 & 1.64354100 & -1.39351700 \\
\hline $\mathrm{C}$ & 1.87107900 & -6.53854900 & 1.07476300 & $\mathrm{C}$ & 2.71122000 & -3.81823800 & -2.83908000 \\
\hline $\mathrm{H}$ & 2.94121400 & -6.74863700 & 0.98322000 & $\mathrm{C}$ & 2.30109600 & -0.64943300 & -4.76401800 \\
\hline $\mathrm{H}$ & 1.33879500 & -7.28374200 & 0.47507600 & $\mathrm{H}$ & 1.20300200 & -0.58022700 & -4.75184600 \\
\hline $\mathrm{H}$ & 1.58405200 & -6.67703800 & 2.12093900 & $\mathrm{H}$ & 2.65557900 & -0.20446200 & -5.69785000 \\
\hline $\mathrm{C}$ & 1.96737800 & -4.96557800 & -0.86305500 & $\mathrm{C}$ & 1.40106800 & 1.96887800 & -3.82000400 \\
\hline $\mathrm{H}$ & 1.69209400 & -3.97815000 & -1.23480500 & $\mathrm{H}$ & 0.69084600 & 1.53486700 & -3.11053900 \\
\hline $\mathrm{H}$ & 1.49827500 & -5.72289500 & -1.50056900 & $\mathrm{H}$ & 1.36061500 & 3.05700500 & -3.72279700 \\
\hline $\mathrm{H}$ & 3.05089700 & -5.07658700 & -0.96609000 & $\mathrm{H}$ & 1.06583400 & 1.72536400 & -4.83315000 \\
\hline $\mathrm{C}$ & 0.00000000 & -4.96708300 & 0.65396800 & $\mathrm{C}$ & 5.26093600 & -1.64331600 & 0.68730600 \\
\hline $\mathrm{H}$ & -0.38114100 & -5.21043900 & 1.64702200 & $\mathrm{H}$ & 6.29129000 & -1.72299200 & 0.32753700 \\
\hline $\mathrm{H}$ & -0.46268900 & -5.64437300 & -0.06989200 & $\mathrm{H}$ & 5.31008600 & -1.32497400 & 1.73243700 \\
\hline $\mathrm{H}$ & -0.30204300 & -3.94439800 & 0.41806500 & $\mathrm{H}$ & 4.81772100 & -2.64080500 & 0.66036100 \\
\hline $\mathrm{C}$ & 3.21074100 & -1.10464700 & 3.65047300 & $\mathrm{C}$ & 3.76294800 & 2.04594100 & -4.63175100 \\
\hline $\mathrm{C}$ & 3.60798700 & -0.00670100 & 2.65818600 & $\mathrm{H}$ & 3.46607800 & 1.75342000 & -5.64285200 \\
\hline $\mathrm{H}$ & 4.57331200 & -0.21502300 & 2.18789700 & $\mathrm{H}$ & 3.75367500 & 3.13959000 & -4.59708900 \\
\hline $\mathrm{H}$ & 3.69601600 & 0.95950100 & 3.16546200 & $\mathrm{H}$ & 4.78828200 & 1.70485800 & -4.46826800 \\
\hline
\end{tabular}




\begin{tabular}{|c|c|c|c|c|c|c|c|}
\hline $\mathrm{H}$ & 2.85563000 & 0.07987200 & 1.87126500 & $\mathrm{C}$ & 6.51956000 & -0.21001800 & -2.28975300 \\
\hline $\mathrm{C}$ & 4.32518700 & -1.28052400 & 4.68809200 & $\mathrm{H}$ & 6.13726200 & 0.70645200 & -2.74455000 \\
\hline $\mathrm{H}$ & 4.07784800 & -2.08662700 & 5.38505800 & $\mathrm{H}$ & 6.87303100 & 0.03452200 & -1.28414900 \\
\hline $\mathrm{H}$ & 4.45341100 & -0.35612000 & 5.26020500 & $\mathrm{H}$ & 7.38841100 & -0.53509800 & -2.87019900 \\
\hline $\mathrm{H}$ & 5.29058300 & -1.51049700 & 4.22803500 & $\mathrm{C}$ & 1.29631000 & -4.36521300 & -3.04279200 \\
\hline $\mathrm{C}$ & 1.94767300 & -0.66960100 & 4.38960900 & $\mathrm{H}$ & 0.97834000 & -4.28674600 & -4.08660000 \\
\hline $\mathrm{H}$ & 1.12810500 & -0.48870900 & 3.69215300 & $\mathrm{H}$ & 1.24110400 & -5.42167500 & -2.76057100 \\
\hline $\mathrm{H}$ & 2.14320400 & 0.26039300 & 4.93068000 & $\mathrm{H}$ & 0.59418100 & -3.79939100 & -2.42960500 \\
\hline $\mathrm{H}$ & 1.62944800 & -1.42430300 & 5.11096600 & $\mathrm{C}$ & 3.70464400 & -4.63185600 & -3.67549100 \\
\hline $\mathrm{C}$ & 1.16475300 & -4.20343400 & 5.67860700 & $\mathrm{H}$ & 4.71704100 & -4.23000300 & -3.57667900 \\
\hline $\mathrm{H}$ & 0.25931000 & -3.81709300 & 6.15672100 & $\mathrm{H}$ & 3.71676900 & -5.67036500 & -3.33243600 \\
\hline $\mathrm{H}$ & 1.95864700 & -3.46469300 & 5.80715000 & $\mathrm{H}$ & 3.44270400 & -4.64274200 & -4.73711000 \\
\hline $\mathrm{H}$ & 1.45692400 & -5.11254600 & 6.21582500 & $\mathrm{C}$ & 5.46248600 & -1.30515100 & -2.28150300 \\
\hline $\mathrm{C}$ & 0.94924500 & -4.51129400 & 4.20160500 & $\mathrm{H}$ & 5.91374100 & -2.23775400 & -1.90950300 \\
\hline $\mathrm{H}$ & 1.88844200 & -4.86727800 & 3.77565500 & $\mathrm{H}$ & 5.15313800 & -1.50842700 & -3.30793400 \\
\hline $\mathrm{H}$ & 0.24105800 & -5.35127900 & 4.11184100 & $\mathrm{C}$ & 3.09073800 & -3.96743800 & -1.36976500 \\
\hline $\mathrm{C}$ & -0.75963600 & -2.77447100 & 3.75510000 & $\mathrm{H}$ & 2.46399600 & -3.34130400 & -0.73184200 \\
\hline $\mathrm{H}$ & -0.96187700 & -2.03340200 & 2.97921300 & $\mathrm{H}$ & 2.95792900 & -5.00946700 & -1.06494100 \\
\hline $\mathrm{H}$ & -0.66488600 & -2.21874300 & 4.70226400 & $\mathrm{H}$ & 4.13441400 & -3.69591000 & -1.20447000 \\
\hline $\mathrm{C}$ & -1.93126600 & -3.74058700 & 3.86554500 & $\mathrm{~N}$ & 1.58295300 & -0.86796700 & -1.23556400 \\
\hline $\mathrm{H}$ & -2.85182900 & -3.18685400 & 4.07604800 & $\mathrm{C}$ & 2.72877500 & -2.10074800 & -4.66423900 \\
\hline $\mathrm{H}$ & -1.80191500 & -4.47058700 & 4.67183000 & $\mathrm{H}$ & 3.71893700 & -2.23758300 & -5.12702500 \\
\hline $\mathrm{H}$ & -2.07711500 & -4.29037400 & 2.93268800 & $\mathrm{H}$ & 2.02215400 & -2.74461200 & -5.19939100 \\
\hline
\end{tabular}

\section{Tb L L-edge XANES}

Table S17. Summary of fit parameters for Tb $\mathrm{L}_{3}$-edge XANES of 3 and 4.

\begin{tabular}{lllllcl} 
Complex & Peak 1 & \multicolumn{3}{c}{ Peak 2 } & \multicolumn{3}{c}{ Peak 3 } & \\
& Intensity & $\begin{array}{l}\text { Energy } \\
(\mathrm{eV})\end{array}$ & Intensity & $\begin{array}{l}\text { Energy } \\
(\mathrm{eV})\end{array}$ & Intensity & $\begin{array}{l}\text { Energy } \\
(\mathrm{eV})\end{array}$ \\
$\mathbf{3}$ & $4.34(4)$ & $7519.20(4)$ & $\mathrm{NA}$ & $\mathrm{NA}$ & $\mathrm{NA}$ & $\mathrm{NA}$ \\
$\mathbf{4}$ & $1.84(3)$ & $7512.0(2)$ & $3.48(3)$ & $7520.30(3)$ & $4.25(5)$ & $7528.50(3)$
\end{tabular}




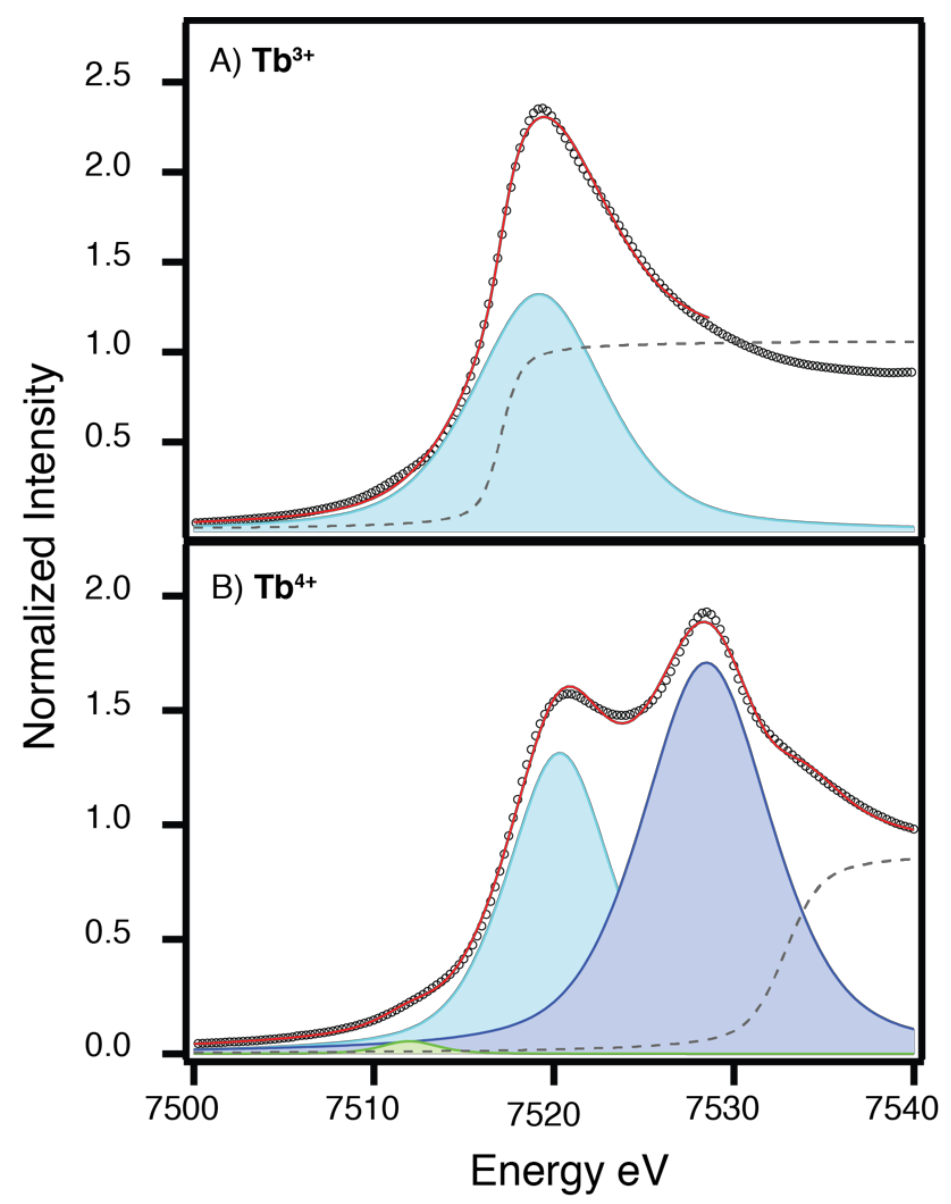

Figure S46. Tb $\mathrm{L}_{3}$-edge XAS experimental data (black) obtained for 3(A) and 4 (B) and the pseudo-Voigt [blue (p2) and purple (p3)] and steplike functions (gray dashed line), which sum to generate the curve fit (red). The shoulder feature observed near the edge onset has little intensity in $\mathbf{4}$ and is modeled with a single function [green (p1)] that is barely visible in the baseline of the spectrum.

Table S18. Normalized and Background Subtracted $\mathrm{L}_{3}$-edge XAS of 3 and 4.

$\begin{array}{rrrr}\text { 3_TbL3_eV } & \text { 3_TbL3_Int } & \text { 4_TbL3_eV } & \text { 3_TbL3_Int } \\ 6881.881 & 8.99 \mathrm{E}-03 & 6881.74 & 8.25 \mathrm{E}-03 \\ 6891.8785 & 8.03 \mathrm{E}-03 & 6891.7375 & 7.77 \mathrm{E}-03 \\ 6901.882 & 7.67 \mathrm{E}-03 & 6901.741 & 7.26 \mathrm{E}-03 \\ 6911.8815 & 7.37 \mathrm{E}-03 & 6911.7405 & 6.54 \mathrm{E}-03 \\ 6921.878 & 6.50 \mathrm{E}-03 & 6921.737 & 6.50 \mathrm{E}-03 \\ 6931.8795 & 6.22 \mathrm{E}-03 & 6931.7385 & 6.81 \mathrm{E}-03 \\ 6941.882 & 5.29 \mathrm{E}-03 & 6941.741 & 4.98 \mathrm{E}-03 \\ 6951.88 & 5.73 \mathrm{E}-03 & 6951.739 & 5.36 \mathrm{E}-03 \\ 6961.8785 & 6.23 \mathrm{E}-03 & 6961.7375 & 5.94 \mathrm{E}-03 \\ 6971.882 & 4.87 \mathrm{E}-03 & 6971.741 & 4.40 \mathrm{E}-03 \\ 6981.881 & 5.14 \mathrm{E}-03 & 6981.74 & 6.52 \mathrm{E}-03\end{array}$




\begin{tabular}{|c|c|c|c|}
\hline 6991.8795 & $5.39 \mathrm{E}-03$ & 6991.7385 & 7.08E-03 \\
\hline 7001.8824 & $5.10 \mathrm{E}-03$ & 7001.7414 & $7.04 \mathrm{E}-03$ \\
\hline 7011.88 & $5.19 \mathrm{E}-03$ & 7011.739 & 7.38E-03 \\
\hline 7021.8815 & 4.40E-03 & 7021.7405 & $8.10 \mathrm{E}-03$ \\
\hline 7031.8776 & 4.12E-03 & 7031.7366 & $8.05 \mathrm{E}-03$ \\
\hline 7041.8776 & $3.92 \mathrm{E}-03$ & 7041.7366 & $7.82 \mathrm{E}-03$ \\
\hline 7051.881 & 3.99E-03 & 7051.74 & $7.45 \mathrm{E}-03$ \\
\hline 7061.8785 & 4.14E-03 & 7061.7375 & 7.43E-03 \\
\hline 7071.879 & 4.54E-03 & 7071.738 & 7.54E-03 \\
\hline 7081.878 & $3.69 \mathrm{E}-03$ & 7081.737 & 7.04E-03 \\
\hline 7091.88 & $3.56 \mathrm{E}-03$ & 7091.739 & $7.41 \mathrm{E}-03$ \\
\hline 7092.132 & 3.99E-03 & 7091.991 & 7.64E-03 \\
\hline 7092.3785 & 4.44E-03 & 7092.2375 & $8.15 \mathrm{E}-03$ \\
\hline 7092.6305 & 4.07E-03 & 7092.4895 & $7.81 \mathrm{E}-03$ \\
\hline 7092.8776 & 4.24E-03 & 7092.7366 & $7.75 \mathrm{E}-03$ \\
\hline 7093.1295 & 4.39E-03 & 7092.9885 & $7.68 \mathrm{E}-03$ \\
\hline 7093.3815 & 4.35E-03 & 7093.2405 & $7.80 \mathrm{E}-03$ \\
\hline 7093.6285 & 4.31E-03 & 7093.4875 & 7.91E-03 \\
\hline 7093.8805 & 4.32E-03 & 7093.7395 & 7.90E-03 \\
\hline 7094.1324 & $4.08 \mathrm{E}-03$ & 7093.9914 & $8.11 \mathrm{E}-03$ \\
\hline 7094.3795 & $4.21 \mathrm{E}-03$ & 7094.2385 & $8.00 \mathrm{E}-03$ \\
\hline 7094.6315 & 4.20E-03 & 7094.4905 & 8.00E-03 \\
\hline 7094.8785 & 4.21E-03 & 7094.7375 & 7.93E-03 \\
\hline 7095.1305 & 4.24E-03 & 7094.9895 & 7.87E-03 \\
\hline 7095.378 & $4.40 \mathrm{E}-03$ & 7095.237 & 8.32E-03 \\
\hline 7095.63 & 4.32E-03 & 7095.489 & $8.06 \mathrm{E}-03$ \\
\hline 7095.882 & $4.53 \mathrm{E}-03$ & 7095.741 & 8.01E-03 \\
\hline 7096.129 & 4.18E-03 & 7095.988 & $8.18 \mathrm{E}-03$ \\
\hline 7096.3815 & 4.30E-03 & 7096.2405 & $8.07 \mathrm{E}-03$ \\
\hline 7096.6285 & $4.55 \mathrm{E}-03$ & 7096.4875 & 8.08E-03 \\
\hline 7096.881 & 4.41E-03 & 7096.74 & $7.80 \mathrm{E}-03$ \\
\hline 7097.128 & 4.32E-03 & 7096.987 & 8.09E-03 \\
\hline 7097.3805 & 4.77E-03 & 7097.2395 & 7.38E-03 \\
\hline 7097.6276 & 4.59E-03 & 7097.4866 & $7.22 \mathrm{E}-03$ \\
\hline 7097.88 & 4.10E-03 & 7097.739 & $6.93 \mathrm{E}-03$ \\
\hline 7098.1324 & $3.86 \mathrm{E}-03$ & 7097.9914 & $7.14 \mathrm{E}-03$ \\
\hline 7098.3795 & 3.94E-03 & 7098.2385 & $7.42 \mathrm{E}-03$ \\
\hline 7098.632 & $3.96 \mathrm{E}-03$ & 7098.491 & 7.33E-03 \\
\hline 7098.8795 & 4.09E-03 & 7098.7385 & $7.40 \mathrm{E}-03$ \\
\hline 7099.132 & 4.30E-03 & 7098.991 & $6.90 \mathrm{E}-03$ \\
\hline
\end{tabular}




$\begin{array}{rrrr}7099.3795 & 4.08 \mathrm{E}-03 & 7099.2385 & 6.33 \mathrm{E}-03 \\ 7099.632 & 4.11 \mathrm{E}-03 & 7099.491 & 6.32 \mathrm{E}-03 \\ 7099.8795 & 4.27 \mathrm{E}-03 & 7099.7385 & 6.21 \mathrm{E}-03 \\ 7100.132 & 4.55 \mathrm{E}-03 & 7099.991 & 6.50 \mathrm{E}-03 \\ 7100.3795 & 3.67 \mathrm{E}-03 & 7100.2385 & 6.57 \mathrm{E}-03 \\ 7100.632 & 3.18 \mathrm{E}-03 & 7100.491 & 6.69 \mathrm{E}-03 \\ 7100.8795 & 3.75 \mathrm{E}-03 & 7100.7385 & 6.37 \mathrm{E}-03 \\ 7101.132 & 3.83 \mathrm{E}-03 & 7100.991 & 6.87 \mathrm{E}-03 \\ 7101.3795 & 3.69 \mathrm{E}-03 & 7101.2385 & 7.53 \mathrm{E}-03 \\ 7101.6324 & 3.74 \mathrm{E}-03 & 7101.4914 & 7.77 \mathrm{E}-03 \\ 7101.88 & 3.81 \mathrm{E}-03 & 7101.739 & 8.16 \mathrm{E}-03 \\ 7102.1276 & 5.05 \mathrm{E}-03 & 7101.9866 & 7.90 \mathrm{E}-03 \\ 7102.3805 & 5.13 \mathrm{E}-03 & 7102.2395 & 7.50 \mathrm{E}-03 \\ 7102.628 & 5.01 \mathrm{E}-03 & 7102.487 & 6.77 \mathrm{E}-03 \\ 7102.8805 & 5.07 \mathrm{E}-03 & 7102.7395 & 6.75 \mathrm{E}-03 \\ 7103.1285 & 5.07 \mathrm{E}-03 & 7102.9875 & 6.50 \mathrm{E}-03 \\ 7103.381 & 5.02 \mathrm{E}-03 & 7103.24 & 6.60 \mathrm{E}-03 \\ 7103.629 & 4.76 \mathrm{E}-03 & 7103.488 & 6.61 \mathrm{E}-03 \\ 7103.882 & 4.05 \mathrm{E}-03 & 7103.741 & 6.53 \mathrm{E}-03 \\ 7104.1295 & 4.44 \mathrm{E}-03 & 7103.9885 & 6.36 \mathrm{E}-03 \\ 7104.3776 & 4.55 \mathrm{E}-03 & 7104.2366 & 6.45 \mathrm{E}-03 \\ 7104.6305 & 4.83 \mathrm{E}-03 & 7104.4895 & 5.68 \mathrm{E}-03 \\ 7104.8785 & 4.82 \mathrm{E}-03 & 7104.7375 & 5.64 \mathrm{E}-03 \\ 7105.131 & 4.77 \mathrm{E}-03 & 7104.99 & 5.79 \mathrm{E}-03 \\ 7105.379 & 4.79 \mathrm{E}-03 & 7105.238 & 5.62 \mathrm{E}-03 \\ 7105.632 & 4.67 \mathrm{E}-03 & 7105.491 & 5.24 \mathrm{E}-03 \\ 7105.88 & 4.58 \mathrm{E}-03 & 7105.739 & 5.57 \mathrm{E}-03 \\ 7106.128 & 4.75 \mathrm{E}-03 & 7105.987 & 5.65 \mathrm{E}-03 \\ 7106.381 & 4.62 \mathrm{E}-03 & 7106.24 & 4.86 \mathrm{E}-03 \\ 7106.629 & 4.62 \mathrm{E}-03 & 7106.488 & 4.37 \mathrm{E}-03 \\ 7106.882 & 4.67 \mathrm{E}-03 & 7106.741 & 4.38 \mathrm{E}-03 \\ 7107.1305 & 4.40 \mathrm{E}-03 & 7106.9895 & 4.94 \mathrm{E}-03 \\ 7107.3785 & 3.89 \mathrm{E}-03 & 7107.2375 & 4.60 \mathrm{E}-03 \\ 7107.6315 & 3.90 \mathrm{E}-03 & 7107.4905 & 4.31 \mathrm{E}-03 \\ 7107.8795 & 3.95 \mathrm{E}-03 & 7107.7385 & 4.62 \mathrm{E}-03 \\ 7108.128 & 3.85 \mathrm{E}-03 & 7107.987 & 4.52 \mathrm{E}-03 \\ 7108.381 & 3.81 \mathrm{E}-03 & 7108.24 & 4.96 \mathrm{E}-03 \\ 7108.629 & 3.85 \mathrm{E}-03 & 7108.488 & 4.36 \mathrm{E}-03 \\ 7.39 \mathrm{E}-03 & 7108.7414 & 4.44 \mathrm{E}-03 \\ 7.305 & 5.44 \mathrm{E}-03 & 7108.9895 & 3.99 \mathrm{E}-03\end{array}$




$\begin{array}{rrrr}7109.379 & 5.88 \mathrm{E}-03 & 7109.238 & 4.08 \mathrm{E}-03 \\ 7109.632 & 5.74 \mathrm{E}-03 & 7109.491 & 4.23 \mathrm{E}-03 \\ 7109.8805 & 5.73 \mathrm{E}-03 & 7109.7395 & 4.06 \mathrm{E}-03 \\ 7110.129 & 5.76 \mathrm{E}-03 & 7109.988 & 4.11 \mathrm{E}-03 \\ 7110.382 & 5.76 \mathrm{E}-03 & 7110.241 & 4.61 \mathrm{E}-03 \\ 7110.6305 & 5.40 \mathrm{E}-03 & 7110.4895 & 4.35 \mathrm{E}-03 \\ 7110.879 & 5.18 \mathrm{E}-03 & 7110.738 & 4.56 \mathrm{E}-03 \\ 7111.1324 & 5.31 \mathrm{E}-03 & 7110.9914 & 4.02 \mathrm{E}-03 \\ 7111.3805 & 5.31 \mathrm{E}-03 & 7111.2395 & 3.84 \mathrm{E}-03 \\ 7111.629 & 6.20 \mathrm{E}-03 & 7111.488 & 4.13 \mathrm{E}-03 \\ 7111.8776 & 6.47 \mathrm{E}-03 & 7111.7366 & 4.52 \mathrm{E}-03 \\ 7112.131 & 6.50 \mathrm{E}-03 & 7111.99 & 4.79 \mathrm{E}-03 \\ 7112.3795 & 6.47 \mathrm{E}-03 & 7112.2385 & 4.97 \mathrm{E}-03 \\ 7112.628 & 5.67 \mathrm{E}-03 & 7112.487 & 5.42 \mathrm{E}-03 \\ 7112.8815 & 5.73 \mathrm{E}-03 & 7112.7405 & 5.35 \mathrm{E}-03 \\ 7113.1305 & 5.84 \mathrm{E}-03 & 7112.9895 & 5.73 \mathrm{E}-03 \\ 7113.379 & 6.15 \mathrm{E}-03 & 7113.238 & 5.58 \mathrm{E}-03 \\ 7113.6324 & 6.15 \mathrm{E}-03 & 7113.4914 & 4.67 \mathrm{E}-03 \\ 7113.881 & 6.79 \mathrm{E}-03 & 7113.74 & 5.22 \mathrm{E}-03 \\ 7114.1295 & 6.96 \mathrm{E}-03 & 7113.9885 & 4.30 \mathrm{E}-03 \\ 7114.3785 & 6.98 \mathrm{E}-03 & 7114.2375 & 4.39 \mathrm{E}-03 \\ 7114.632 & 6.76 \mathrm{E}-03 & 7114.491 & 5.44 \mathrm{E}-03 \\ 7114.881 & 5.92 \mathrm{E}-03 & 7114.74 & 5.01 \mathrm{E}-03 \\ 7115.1295 & 6.08 \mathrm{E}-03 & 7114.9885 & 4.76 \mathrm{E}-03 \\ 7115.3785 & 5.86 \mathrm{E}-03 & 7115.2375 & 6.18 \mathrm{E}-03 \\ 7115.632 & 6.20 \mathrm{E}-03 & 7115.491 & 6.38 \mathrm{E}-03 \\ 7115.881 & 6.09 \mathrm{E}-03 & 7115.74 & 6.52 \mathrm{E}-03 \\ 7116.1295 & 6.22 \mathrm{E}-03 & 7115.9885 & 7.55 \mathrm{E}-03 \\ 7116.3785 & 6.43 \mathrm{E}-03 & 7116.2375 & 8.14 \mathrm{E}-03 \\ 7116.6324 & 6.13 \mathrm{E}-03 & 7116.4914 & 8.47 \mathrm{E}-03 \\ 7116.881 & 5.36 \mathrm{E}-03 & 7116.74 & 8.77 \mathrm{E}-03 \\ 7117.13 & 5.54 \mathrm{E}-03 & 7116.989 & 8.74 \mathrm{E}-03 \\ 7117.379 & 6.23 \mathrm{E}-03 & 7117.238 & 7.78 \mathrm{E}-03 \\ 7117.628 & 6.30 \mathrm{E}-03 & 7117.487 & 7.97 \mathrm{E}-03 \\ 7117.882 & 6.33 \mathrm{E}-03 & 7117.741 & 7.84 \mathrm{E}-03 \\ 7118.131 & 6.29 \mathrm{E}-03 & 7117.99 & 8.19 \mathrm{E}-03 \\ 7118.38 & 6.43 \mathrm{E}-03 & 7118.239 & 8.19 \mathrm{E}-03 \\ 7118.629 & 6.60 \mathrm{E}-03 & 7118.488 & 7.93 \mathrm{E}-03 \\ 7118.878 & 6.60 \mathrm{E}-03 & 7118.737 & 7.45 \mathrm{E}-03 \\ 7.132 & 6.45 \mathrm{E}-03 & 7118.991 & 7.25 \mathrm{E}-03\end{array}$




$\begin{array}{rrrr}7119.381 & 6.45 \mathrm{E}-03 & 7119.24 & 7.75 \mathrm{E}-03 \\ 7119.63 & 6.53 \mathrm{E}-03 & 7119.489 & 6.59 \mathrm{E}-03 \\ 7119.8795 & 6.74 \mathrm{E}-03 & 7119.7385 & 6.44 \mathrm{E}-03 \\ 7120.1285 & 6.88 \mathrm{E}-03 & 7119.9875 & 7.33 \mathrm{E}-03 \\ 7120.3776 & 7.00 \mathrm{E}-03 & 7120.2366 & 7.75 \mathrm{E}-03 \\ 7120.632 & 6.67 \mathrm{E}-03 & 7120.491 & 8.26 \mathrm{E}-03 \\ 7120.881 & 6.28 \mathrm{E}-03 & 7120.74 & 8.52 \mathrm{E}-03 \\ 7121.13 & 6.88 \mathrm{E}-03 & 7120.989 & 8.23 \mathrm{E}-03 \\ 7121.3795 & 6.58 \mathrm{E}-03 & 7121.2385 & 8.15 \mathrm{E}-03 \\ 7121.6285 & 6.53 \mathrm{E}-03 & 7121.4875 & 8.10 \mathrm{E}-03 \\ 7121.878 & 6.54 \mathrm{E}-03 & 7121.737 & 8.13 \mathrm{E}-03 \\ 7122.132 & 6.63 \mathrm{E}-03 & 7121.991 & 8.16 \mathrm{E}-03 \\ 7122.3815 & 6.48 \mathrm{E}-03 & 7122.2405 & 8.39 \mathrm{E}-03 \\ 7122.631 & 6.20 \mathrm{E}-03 & 7122.49 & 8.66 \mathrm{E}-03 \\ 7122.8805 & 6.37 \mathrm{E}-03 & 7122.7395 & 8.69 \mathrm{E}-03 \\ 7123.1295 & 6.50 \mathrm{E}-03 & 7122.9885 & 8.74 \mathrm{E}-03 \\ 7123.379 & 6.78 \mathrm{E}-03 & 7123.238 & 8.69 \mathrm{E}-03 \\ 7123.6285 & 6.78 \mathrm{E}-03 & 7123.4875 & 8.72 \mathrm{E}-03 \\ 7123.878 & 6.95 \mathrm{E}-03 & 7123.737 & 9.89 \mathrm{E}-03 \\ 7124.1324 & 7.05 \mathrm{E}-03 & 7123.9914 & 9.88 \mathrm{E}-03 \\ 7124.382 & 7.07 \mathrm{E}-03 & 7124.241 & 1.01 \mathrm{E}-02 \\ 7124.6315 & 7.11 \mathrm{E}-03 & 7124.4905 & 1.03 \mathrm{E}-02 \\ 7124.881 & 7.80 \mathrm{E}-03 & 7124.74 & 9.90 \mathrm{E}-03 \\ 7125.1305 & 7.71 \mathrm{E}-03 & 7124.9895 & 9.72 \mathrm{E}-03 \\ 7125.38 & 8.25 \mathrm{E}-03 & 7125.239 & 9.71 \mathrm{E}-03 \\ 7125.6295 & 8.84 \mathrm{E}-03 & 7125.4885 & 9.82 \mathrm{E}-03 \\ 7125.879 & 8.82 \mathrm{E}-03 & 7125.738 & 9.90 \mathrm{E}-03 \\ 7126.129 & 8.99 \mathrm{E}-03 & 7125.988 & 1.01 \mathrm{E}-02 \\ 7126.3785 & 8.84 \mathrm{E}-03 & 7126.2375 & 1.12 \mathrm{E}-02 \\ 7126.628 & 8.87 \mathrm{E}-03 & 7126.487 & 1.17 \mathrm{E}-02 \\ 7126.878 & 8.99 \mathrm{E}-03 & 7126.737 & 1.14 \mathrm{E}-02 \\ 7127.1324 & 9.25 \mathrm{E}-03 & 7126.9914 & 1.16 \mathrm{E}-02 \\ 7127.382 & 9.22 \mathrm{E}-03 & 7127.241 & 1.22 \mathrm{E}-02 \\ 7127.632 & 9.36 \mathrm{E}-03 & 7127.491 & 1.14 \mathrm{E}-02 \\ 7127.8815 & 9.35 \mathrm{E}-03 & 7127.7405 & 1.23 \mathrm{E}-02 \\ 7128.1315 & 9.29 \mathrm{E}-03 & 7127.9905 & 1.23 \mathrm{E}-02 \\ 7128.3815 & 9.46 \mathrm{E}-03 & 7128.2405 & 1.26 \mathrm{E}-02 \\ 7128.631 & 9.72 \mathrm{E}-03 & 7128.49 & 1.27 \mathrm{E}-02 \\ 7128.881 & 9.77 \mathrm{E}-03 & 7128.74 & 1.26 \mathrm{E}-02 \\ 7129.131 & 1.03 \mathrm{E}-02 & 7128.99 & 1.25 \mathrm{E}-02\end{array}$




$\begin{array}{rrrr}7129.3805 & 1.04 \mathrm{E}-02 & 7129.2395 & 1.27 \mathrm{E}-02 \\ 7129.6305 & 1.05 \mathrm{E}-02 & 7129.4895 & 1.26 \mathrm{E}-02 \\ 7129.8805 & 1.12 \mathrm{E}-02 & 7129.7395 & 1.29 \mathrm{E}-02 \\ 7130.1305 & 1.10 \mathrm{E}-02 & 7129.9895 & 1.41 \mathrm{E}-02 \\ 7130.3805 & 1.11 \mathrm{E}-02 & 7130.2395 & 1.38 \mathrm{E}-02 \\ 7130.6305 & 1.11 \mathrm{E}-02 & 7130.4895 & 1.39 \mathrm{E}-02 \\ 7130.8805 & 1.10 \mathrm{E}-02 & 7130.7395 & 1.37 \mathrm{E}-02 \\ 7131.1305 & 1.06 \mathrm{E}-02 & 7130.9895 & 1.36 \mathrm{E}-02 \\ 7131.3805 & 1.12 \mathrm{E}-02 & 7131.2395 & 1.35 \mathrm{E}-02 \\ 7131.6305 & 1.11 \mathrm{E}-02 & 7131.4895 & 1.38 \mathrm{E}-02 \\ 7131.8805 & 1.12 \mathrm{E}-02 & 7131.7395 & 1.42 \mathrm{E}-02 \\ 7132.131 & 1.08 \mathrm{E}-02 & 7131.99 & 1.37 \mathrm{E}-02 \\ 7132.381 & 1.08 \mathrm{E}-02 & 7132.24 & 1.37 \mathrm{E}-02 \\ 7132.631 & 1.10 \mathrm{E}-02 & 7132.49 & 1.40 \mathrm{E}-02 \\ 7132.8815 & 1.07 \mathrm{E}-02 & 7132.7405 & 1.39 \mathrm{E}-02 \\ 7133.1315 & 1.08 \mathrm{E}-02 & 7132.9905 & 1.40 \mathrm{E}-02 \\ 7133.3815 & 1.07 \mathrm{E}-02 & 7133.2405 & 1.39 \mathrm{E}-02 \\ 7133.632 & 1.04 \mathrm{E}-02 & 7133.491 & 1.41 \mathrm{E}-02 \\ 7133.882 & 1.01 \mathrm{E}-02 & 7133.741 & 1.42 \mathrm{E}-02 \\ 7134.1324 & 9.93 \mathrm{E}-03 & 7133.9914 & 1.39 \mathrm{E}-02 \\ 7134.3776 & 9.99 \mathrm{E}-03 & 7134.2366 & 1.42 \mathrm{E}-02 \\ 7134.628 & 9.73 \mathrm{E}-03 & 7134.487 & 1.42 \mathrm{E}-02 \\ 7134.8785 & 9.65 \mathrm{E}-03 & 7134.7375 & 1.59 \mathrm{E}-02 \\ 7135.1285 & 9.66 \mathrm{E}-03 & 7134.9875 & 1.63 \mathrm{E}-02 \\ 7135.379 & 1.06 \mathrm{E}-02 & 7135.238 & 1.64 \mathrm{E}-02 \\ 7135.6295 & 1.10 \mathrm{E}-02 & 7135.4885 & 1.60 \mathrm{E}-02 \\ 7135.88 & 1.07 \mathrm{E}-02 & 7135.739 & 1.53 \mathrm{E}-02 \\ 7138.8305 & 1.03 \mathrm{E}-02 & 7138.4895 & 1.55 \mathrm{E}-02 \\ 7139.132 & 1.04 \mathrm{E}-02 & 7138.991 & 1.53 \mathrm{E}-02\end{array}$




$\begin{array}{rrrr}7139.3776 & 1.07 \mathrm{E}-02 & 7139.2366 & 1.56 \mathrm{E}-02 \\ 7139.6285 & 1.07 \mathrm{E}-02 & 7139.4875 & 1.53 \mathrm{E}-02 \\ 7139.879 & 1.07 \mathrm{E}-02 & 7139.738 & 1.54 \mathrm{E}-02 \\ 7140.13 & 1.06 \mathrm{E}-02 & 7139.989 & 1.54 \mathrm{E}-02 \\ 7140.3805 & 1.07 \mathrm{E}-02 & 7140.2395 & 1.52 \mathrm{E}-02 \\ 7140.6315 & 1.06 \mathrm{E}-02 & 7140.4905 & 1.52 \mathrm{E}-02 \\ 7140.8824 & 1.05 \mathrm{E}-02 & 7140.7414 & 1.52 \mathrm{E}-02 \\ 7141.128 & 9.60 \mathrm{E}-03 & 7140.987 & 1.47 \mathrm{E}-02 \\ 7141.379 & 9.13 \mathrm{E}-03 & 7141.238 & 1.51 \mathrm{E}-02 \\ 7141.63 & 8.86 \mathrm{E}-03 & 7141.489 & 1.46 \mathrm{E}-02 \\ 7141.8805 & 8.75 \mathrm{E}-03 & 7141.7395 & 1.52 \mathrm{E}-02 \\ 7144.878 & 8.71 \mathrm{E}-03 & 7144.737 & 1.43 \mathrm{E}-02 \\ 7147.878 & 8.43 \mathrm{E}-03 & 7147.737 & 1.39 \mathrm{E}-02 \\ 7150.881 & 7.85 \mathrm{E}-03 & 7150.74 & 1.37 \mathrm{E}-02 \\ 7153.882 & 7.47 \mathrm{E}-03 & 7153.741 & 1.33 \mathrm{E}-02 \\ 7156.881 & 8.01 \mathrm{E}-03 & 7156.74 & 1.40 \mathrm{E}-02 \\ 7159.8776 & 7.81 \mathrm{E}-03 & 7159.7366 & 1.36 \mathrm{E}-02 \\ 7162.882 & 7.51 \mathrm{E}-03 & 7162.741 & 1.37 \mathrm{E}-02 \\ 7165.8795 & 7.63 \mathrm{E}-03 & 7165.7385 & 1.36 \mathrm{E}-02 \\ 7168.8795 & 7.27 \mathrm{E}-03 & 7168.7385 & 1.36 \mathrm{E}-02 \\ 7171.878 & 7.13 \mathrm{E}-03 & 7171.737 & 1.34 \mathrm{E}-02 \\ 7174.879 & 7.06 \mathrm{E}-03 & 7174.738 & 1.34 \mathrm{E}-02 \\ 7177.8776 & 7.37 \mathrm{E}-03 & 7177.7366 & 1.32 \mathrm{E}-02 \\ 7180.879 & 6.88 \mathrm{E}-03 & 7180.738 & 1.24 \mathrm{E}-02 \\ 7183.8785 & 6.97 \mathrm{E}-03 & 7183.7375 & 1.26 \mathrm{E}-02 \\ 7186.881 & 6.91 \mathrm{E}-03 & 7186.74 & 1.14 \mathrm{E}-02 \\ 7189.881 & 6.62 \mathrm{E}-03 & 7189.74 & 1.20 \mathrm{E}-02 \\ 7192.879 & 6.72 \mathrm{E}-03 & 7192.738 & 1.19 \mathrm{E}-02 \\ 7195.88 & 6.50 \mathrm{E}-03 & 7195.739 & 1.18 \mathrm{E}-02 \\ 7198.8785 & 6.14 \mathrm{E}-03 & 7198.7375 & 1.15 \mathrm{E}-02 \\ 7201.88 & 5.96 \mathrm{E}-03 & 7201.739 & 1.07 \mathrm{E}-02 \\ 7204.879 & 5.57 \mathrm{E}-03 & 7204.738 & 1.01 \mathrm{E}-02 \\ 7207.881 & 5.50 \mathrm{E}-03 & 7207.74 & 1.08 \mathrm{E}-02 \\ 7210.881 & 5.22 \mathrm{E}-03 & 7210.74 & 1.04 \mathrm{E}-02 \\ 7213.8785 & 5.16 \mathrm{E}-03 & 7213.7375 & 1.06 \mathrm{E}-02 \\ 7216.8785 & 5.15 \mathrm{E}-03 & 7216.7375 & 1.12 \mathrm{E}-02 \\ 728.878 & 5.67 \mathrm{E}-03 & 7219.741 & 1.13 \mathrm{E}-02 \\ 7.35 \mathrm{E}-03 & 7222.737 & 1.04 \mathrm{E}-02 \\ 7.8315 & 4.91 \mathrm{E}-03 & 7225.7405 & 1.18 \mathrm{E}-02 \\ 7.78 \mathrm{E}-03 & 7228.737 & 1.19 \mathrm{E}-02\end{array}$




$\begin{array}{rrrr}7231.8824 & 4.35 \mathrm{E}-03 & 7231.7414 & 1.10 \mathrm{E}-02 \\ 7234.8795 & 3.94 \mathrm{E}-03 & 7234.7385 & 1.09 \mathrm{E}-02 \\ 7237.879 & 4.04 \mathrm{E}-03 & 7237.738 & 1.10 \mathrm{E}-02 \\ 7240.882 & 3.82 \mathrm{E}-03 & 7240.741 & 1.04 \mathrm{E}-02 \\ 7243.882 & 3.16 \mathrm{E}-03 & 7243.741 & 1.04 \mathrm{E}-02 \\ 7246.88 & 2.60 \mathrm{E}-03 & 7246.739 & 1.02 \mathrm{E}-02 \\ 7249.881 & 2.35 \mathrm{E}-03 & 7249.74 & 1.04 \mathrm{E}-02 \\ 7252.8795 & 2.27 \mathrm{E}-03 & 7252.7385 & 9.07 \mathrm{E}-03 \\ 7255.881 & 2.13 \mathrm{E}-03 & 7255.74 & 9.81 \mathrm{E}-03 \\ 7258.8795 & 1.28 \mathrm{E}-03 & 7258.7385 & 9.52 \mathrm{E}-03 \\ 7261.8815 & 1.59 \mathrm{E}-03 & 7261.7405 & 9.83 \mathrm{E}-03 \\ 7264.881 & 6.64 \mathrm{E}-04 & 7264.74 & 9.62 \mathrm{E}-03 \\ 7267.878 & 1.23 \mathrm{E}-05 & 7267.737 & 9.58 \mathrm{E}-03 \\ 7270.878 & -4.08 \mathrm{E}-04 & 7270.737 & 9.02 \mathrm{E}-03 \\ 7273.8805 & -6.83 \mathrm{E}-04 & 7273.7395 & 8.89 \mathrm{E}-03 \\ 7276.881 & -2.38 \mathrm{E}-04 & 7276.74 & 9.69 \mathrm{E}-03 \\ 7279.879 & -6.92 \mathrm{E}-04 & 7279.738 & 1.00 \mathrm{E}-02 \\ 7282.88 & -1.44 \mathrm{E}-04 & 7282.739 & 1.01 \mathrm{E}-02 \\ 7285.878 & -1.46 \mathrm{E}-04 & 7285.737 & 9.78 \mathrm{E}-03 \\ 7288.8795 & -5.94 \mathrm{E}-04 & 7288.7385 & 9.62 \mathrm{E}-03 \\ 7291.878 & -1.76 \mathrm{E}-03 & 7291.737 & 9.38 \mathrm{E}-03 \\ 7294.8795 & -2.14 \mathrm{E}-03 & 7294.7385 & 8.45 \mathrm{E}-03 \\ 7297.879 & -3.42 \mathrm{E}-03 & 7297.738 & 8.97 \mathrm{E}-03 \\ 7300.881 & -4.39 \mathrm{E}-03 & 7300.74 & 8.63 \mathrm{E}-03 \\ 7303.8805 & -4.33 \mathrm{E}-03 & 7303.7395 & 8.42 \mathrm{E}-03 \\ 7306.8824 & -4.40 \mathrm{E}-03 & 7306.7414 & 7.99 \mathrm{E}-03 \\ 7309.8824 & -4.48 \mathrm{E}-03 & 7309.7414 & 7.73 \mathrm{E}-03 \\ 7312.88 & -3.93 \mathrm{E}-03 & 7312.739 & 8.00 \mathrm{E}-03 \\ 7315.88 & -4.10 \mathrm{E}-03 & 7315.739 & 7.75 \mathrm{E}-03 \\ 7318.878 & -4.66 \mathrm{E}-03 & 7318.737 & 7.01 \mathrm{E}-03 \\ 7321.8785 & -4.68 \mathrm{E}-03 & 7321.7375 & 6.28 \mathrm{E}-03 \\ 7324.8815 & -3.69 \mathrm{E}-03 & 7324.7405 & 6.07 \mathrm{E}-03 \\ 7327.8824 & -3.70 \mathrm{E}-03 & 7327.7414 & 6.13 \mathrm{E}-03 \\ 7330.881 & -3.65 \mathrm{E}-03 & 7330.74 & 4.50 \mathrm{E}-03 \\ 7333.882 & -3.04 \mathrm{E}-03 & 7333.741 & 4.52 \mathrm{E}-03 \\ 7336.8805 & -3.14 \mathrm{E}-03 & 7336.7395 & 4.21 \mathrm{E}-03 \\ 7339.882 & -3.39 \mathrm{E}-03 & 7339.741 & 4.03 \mathrm{E}-03 \\ 7348.881 & -3.61 \mathrm{E}-03 & 7342.74 & 4.18 \mathrm{E}-03 \\ 7382 & -2.88 \mathrm{E}-03 & 7348.741 & 3.68 \mathrm{E}-03\end{array}$




$\begin{array}{rrrr}7351.8785 & -3.20 \mathrm{E}-03 & 7351.7375 & 3.77 \mathrm{E}-03 \\ 7354.878 & -3.30 \mathrm{E}-03 & 7354.737 & 3.40 \mathrm{E}-03 \\ 7357.88 & -3.48 \mathrm{E}-03 & 7357.739 & 3.15 \mathrm{E}-03 \\ 7360.8795 & -3.59 \mathrm{E}-03 & 7360.7385 & 3.26 \mathrm{E}-03 \\ 7363.882 & -3.42 \mathrm{E}-03 & 7363.741 & 3.16 \mathrm{E}-03 \\ 7366.882 & -3.68 \mathrm{E}-03 & 7366.741 & 1.77 \mathrm{E}-03 \\ 7369.879 & -4.13 \mathrm{E}-03 & 7369.738 & 1.45 \mathrm{E}-03 \\ 7372.879 & -4.48 \mathrm{E}-03 & 7372.738 & 1.27 \mathrm{E}-03 \\ 7375.882 & -3.94 \mathrm{E}-03 & 7375.741 & 1.14 \mathrm{E}-03 \\ 7378.882 & -4.52 \mathrm{E}-03 & 7378.741 & 9.10 \mathrm{E}-04 \\ 7381.8795 & -4.70 \mathrm{E}-03 & 7381.7385 & 5.43 \mathrm{E}-04 \\ 7384.88 & -5.08 \mathrm{E}-03 & 7384.739 & 5.77 \mathrm{E}-04 \\ 7387.8776 & -5.12 \mathrm{E}-03 & 7387.7366 & 3.57 \mathrm{E}-04 \\ 7390.878 & -5.42 \mathrm{E}-03 & 7390.737 & -3.49 \mathrm{E}-04 \\ 7393.8815 & -5.39 \mathrm{E}-03 & 7393.7405 & -6.08 \mathrm{E}-04 \\ 7396.882 & -5.35 \mathrm{E}-03 & 7396.741 & -5.80 \mathrm{E}-04 \\ 7399.88 & -5.10 \mathrm{E}-03 & 7399.739 & 3.14 \mathrm{E}-04 \\ 7402.8805 & -5.23 \mathrm{E}-03 & 7402.7395 & 3.17 \mathrm{E}-04 \\ 7405.879 & -5.18 \mathrm{E}-03 & 7405.738 & 9.56 \mathrm{E}-04 \\ 7408.8795 & -5.40 \mathrm{E}-03 & 7408.7385 & 3.75 \mathrm{E}-04 \\ 7411.878 & -5.56 \mathrm{E}-03 & 7411.737 & -7.32 \mathrm{E}-04 \\ 7414.8785 & -5.67 \mathrm{E}-03 & 7414.7375 & -6.42 \mathrm{E}-04 \\ 7417.8824 & -5.58 \mathrm{E}-03 & 7417.7414 & -4.53 \mathrm{E}-04 \\ 7420.878 & -5.33 \mathrm{E}-03 & 7420.737 & -6.09 \mathrm{E}-04 \\ 7423.8815 & -4.91 \mathrm{E}-03 & 7423.7405 & 3.41 \mathrm{E}-04 \\ 7426.8776 & -4.81 \mathrm{E}-03 & 7426.7366 & 6.76 \mathrm{E}-04 \\ 7429.881 & -5.03 \mathrm{E}-03 & 7429.74 & 3.73 \mathrm{E}-05 \\ 7432.882 & -5.20 \mathrm{E}-03 & 7432.741 & -1.10 \mathrm{E}-04 \\ 7435.8805 & -5.39 \mathrm{E}-03 & 7435.7395 & 1.12 \mathrm{E}-03 \\ 7438.8815 & -5.20 \mathrm{E}-03 & 7438.7405 & 1.17 \mathrm{E}-03 \\ 7441.88 & -4.66 \mathrm{E}-03 & 7441.739 & 1.43 \mathrm{E}-03 \\ 7444.881 & -4.21 \mathrm{E}-03 & 7444.74 & 1.69 \mathrm{E}-03 \\ 7450.8805 & -3.33 \mathrm{E}-03 & 7450.7395 & 2.25 \mathrm{E}-03 \\ 7453.879 & -3.22 \mathrm{E}-03 & 7453.738 & 2.83 \mathrm{E}-03 \\ 7456.88 & -3.14 \mathrm{E}-03 & 7456.739 & 3.78 \mathrm{E}-03 \\ 7468.8795 & -2.34 \mathrm{E}-03 & 7459.7375 & 4.12 \mathrm{E}-03 \\ 7495 & -1.78 \mathrm{E}-03 & 7462.7385 & 4.92 \mathrm{E}-03 \\ 74.03 & -7.61 \mathrm{E}-04 & 7465.737 & 5.61 \mathrm{E}-03 \\ 7.07 \mathrm{E}-05 & 7468.738 & 6.37 \mathrm{E}-03\end{array}$




$\begin{array}{rrrr}7471.8824 & 1.16 \mathrm{E}-03 & 7471.7414 & 7.19 \mathrm{E}-03 \\ 7474.878 & 2.64 \mathrm{E}-03 & 7474.737 & 8.43 \mathrm{E}-03 \\ 7477.8815 & 4.03 \mathrm{E}-03 & 7477.7405 & 1.06 \mathrm{E}-02 \\ 7480.8824 & 6.23 \mathrm{E}-03 & 7480.7414 & 1.25 \mathrm{E}-02 \\ 7483.8805 & 8.81 \mathrm{E}-03 & 7483.7395 & 1.46 \mathrm{E}-02 \\ 7484.132 & 8.24 \mathrm{E}-03 & 7483.991 & 1.47 \mathrm{E}-02 \\ 7484.3776 & 7.91 \mathrm{E}-03 & 7484.2366 & 1.50 \mathrm{E}-02 \\ 7484.629 & 8.25 \mathrm{E}-03 & 7484.488 & 1.48 \mathrm{E}-02 \\ 7484.8805 & 8.67 \mathrm{E}-03 & 7484.7395 & 1.51 \mathrm{E}-02 \\ 7485.132 & 9.01 \mathrm{E}-03 & 7484.991 & 1.58 \mathrm{E}-02 \\ 7485.3776 & 9.18 \mathrm{E}-03 & 7485.2366 & 1.59 \mathrm{E}-02 \\ 7485.629 & 9.64 \mathrm{E}-03 & 7485.488 & 1.62 \mathrm{E}-02 \\ 7485.8805 & 9.75 \mathrm{E}-03 & 7485.7395 & 1.65 \mathrm{E}-02 \\ 7486.132 & 1.03 \mathrm{E}-02 & 7485.991 & 1.65 \mathrm{E}-02 \\ 7486.378 & 1.05 \mathrm{E}-02 & 7486.237 & 1.69 \mathrm{E}-02 \\ 7486.6295 & 1.06 \mathrm{E}-02 & 7486.4885 & 1.68 \mathrm{E}-02 \\ 7486.881 & 1.07 \mathrm{E}-02 & 7486.74 & 1.76 \mathrm{E}-02 \\ 7487.1271 & 1.14 \mathrm{E}-02 & 7486.9861 & 1.78 \mathrm{E}-02 \\ 7487.379 & 1.16 \mathrm{E}-02 & 7487.238 & 1.80 \mathrm{E}-02 \\ 7487.6305 & 1.20 \mathrm{E}-02 & 7487.4895 & 1.85 \mathrm{E}-02 \\ 7487.882 & 1.23 \mathrm{E}-02 & 7487.741 & 1.87 \mathrm{E}-02 \\ 7488.128 & 1.27 \mathrm{E}-02 & 7487.987 & 1.87 \mathrm{E}-02 \\ 7488.38 & 1.32 \mathrm{E}-02 & 7488.239 & 1.89 \mathrm{E}-02 \\ 7488.6315 & 1.37 \mathrm{E}-02 & 7488.4905 & 1.91 \mathrm{E}-02 \\ 7488.8776 & 1.40 \mathrm{E}-02 & 7488.7366 & 1.93 \mathrm{E}-02 \\ 7489.1295 & 1.41 \mathrm{E}-02 & 7488.9885 & 1.94 \mathrm{E}-02 \\ 7489.381 & 1.48 \mathrm{E}-02 & 7489.24 & 2.02 \mathrm{E}-02 \\ 7489.6276 & 1.49 \mathrm{E}-02 & 7489.4866 & 2.05 \mathrm{E}-02 \\ 7489.879 & 1.53 \mathrm{E}-02 & 7489.738 & 2.07 \mathrm{E}-02 \\ 7490.131 & 1.53 \mathrm{E}-02 & 7489.99 & 2.06 \mathrm{E}-02 \\ 7490.3829 & 1.55 \mathrm{E}-02 & 7490.2419 & 2.14 \mathrm{E}-02 \\ 7490.629 & 1.62 \mathrm{E}-02 & 7490.488 & 2.16 \mathrm{E}-02 \\ 7490.881 & 1.55 \mathrm{E}-02 & 7490.74 & 2.21 \mathrm{E}-02 \\ 7491.1271 & 1.61 \mathrm{E}-02 & 7490.9861 & 2.25 \mathrm{E}-02 \\ 7491.379 & 1.66 \mathrm{E}-02 & 7491.238 & 2.29 \mathrm{E}-02 \\ 7491.631 & 1.70 \mathrm{E}-02 & 7491.49 & 2.32 \mathrm{E}-02 \\ 7491.8776 & 1.78 \mathrm{E}-02 & 7491.7366 & 2.41 \mathrm{E}-02 \\ 7492.3815 & 1.87 \mathrm{E}-02 & 7492.2405 & 2.48 \mathrm{E}-02 \\ 7.928 & 1.91 \mathrm{E}-02 & 7492.487 & 2.52 \mathrm{E}-02\end{array}$




$\begin{array}{rrrr}7492.88 & 1.95 \mathrm{E}-02 & 7492.739 & 2.56 \mathrm{E}-02 \\ 7493.132 & 2.05 \mathrm{E}-02 & 7492.991 & 2.65 \mathrm{E}-02 \\ 7493.3785 & 2.12 \mathrm{E}-02 & 7493.2375 & 2.68 \mathrm{E}-02 \\ 7493.6305 & 2.18 \mathrm{E}-02 & 7493.4895 & 2.68 \mathrm{E}-02 \\ 7493.8824 & 2.27 \mathrm{E}-02 & 7493.7414 & 2.67 \mathrm{E}-02 \\ 7494.129 & 2.32 \mathrm{E}-02 & 7493.988 & 2.77 \mathrm{E}-02 \\ 7494.381 & 2.35 \mathrm{E}-02 & 7494.24 & 2.99 \mathrm{E}-02 \\ 7494.6276 & 2.42 \mathrm{E}-02 & 7494.4866 & 2.95 \mathrm{E}-02 \\ 7494.88 & 2.50 \mathrm{E}-02 & 7494.739 & 2.96 \mathrm{E}-02 \\ 7495.132 & 2.57 \mathrm{E}-02 & 7494.991 & 3.03 \mathrm{E}-02 \\ 7495.3785 & 2.66 \mathrm{E}-02 & 7495.2375 & 3.13 \mathrm{E}-02 \\ 7495.631 & 2.71 \mathrm{E}-02 & 7495.49 & 3.22 \mathrm{E}-02 \\ 7495.8776 & 2.76 \mathrm{E}-02 & 7495.7366 & 3.23 \mathrm{E}-02 \\ 7496.13 & 2.94 \mathrm{E}-02 & 7495.989 & 3.25 \mathrm{E}-02 \\ 7496.382 & 3.02 \mathrm{E}-02 & 7496.241 & 3.20 \mathrm{E}-02 \\ 7496.629 & 3.10 \mathrm{E}-02 & 7496.488 & 3.31 \mathrm{E}-02 \\ 7496.881 & 3.20 \mathrm{E}-02 & 7496.74 & 3.52 \mathrm{E}-02 \\ 7497.128 & 3.39 \mathrm{E}-02 & 7496.987 & 3.56 \mathrm{E}-02 \\ 7497.3805 & 3.55 \mathrm{E}-02 & 7497.2395 & 3.62 \mathrm{E}-02 \\ 7497.6324 & 3.70 \mathrm{E}-02 & 7497.4914 & 3.70 \mathrm{E}-02 \\ 7497.8795 & 3.80 \mathrm{E}-02 & 7497.7385 & 3.77 \mathrm{E}-02 \\ 7498.132 & 3.91 \mathrm{E}-02 & 7497.991 & 3.85 \mathrm{E}-02 \\ 7498.3785 & 4.10 \mathrm{E}-02 & 7498.2375 & 3.90 \mathrm{E}-02 \\ 7498.631 & 4.19 \mathrm{E}-02 & 7498.49 & 3.96 \mathrm{E}-02 \\ 7498.878 & 4.32 \mathrm{E}-02 & 7498.737 & 4.04 \mathrm{E}-02 \\ 7499.1305 & 4.46 \mathrm{E}-02 & 7498.9895 & 4.07 \mathrm{E}-02 \\ 7499.3776 & 4.61 \mathrm{E}-02 & 7499.2366 & 4.15 \mathrm{E}-02 \\ 7499.63 & 4.73 \mathrm{E}-02 & 7499.489 & 4.24 \mathrm{E}-02 \\ 7499.8824 & 4.87 \mathrm{E}-02 & 7499.7414 & 4.33 \mathrm{E}-02 \\ 7500.1295 & 5.03 \mathrm{E}-02 & 7499.9885 & 4.41 \mathrm{E}-02 \\ 7500.382 & 5.18 \mathrm{E}-02 & 7500.241 & 4.50 \mathrm{E}-02 \\ 7500.629 & 5.33 \mathrm{E}-02 & 7500.488 & 4.60 \mathrm{E}-02 \\ 7500.882 & 5.46 \mathrm{E}-02 & 7500.741 & 4.67 \mathrm{E}-02 \\ 7501.129 & 5.64 \mathrm{E}-02 & 7500.988 & 4.74 \mathrm{E}-02 \\ 7501.3815 & 5.78 \mathrm{E}-02 & 7501.2405 & 4.86 \mathrm{E}-02 \\ 7501.6285 & 5.95 \mathrm{E}-02 & 7501.4875 & 4.93 \mathrm{E}-02 \\ 7501.8815 & 6.15 \mathrm{E}-02 & 7501.7405 & 5.07 \mathrm{E}-02 \\ 7502.1285 & 6.34 \mathrm{E}-02 & 7501.9875 & 5.22 \mathrm{E}-02 \\ 7502.381 & 6.55 \mathrm{E}-02 & 7502.24 & 5.22 \mathrm{E}-02 \\ 7502.6285 & 6.74 \mathrm{E}-02 & 7502.4875 & 5.32 \mathrm{E}-02\end{array}$




$\begin{array}{rrrr}7502.881 & 6.95 \mathrm{E}-02 & 7502.74 & 5.44 \mathrm{E}-02 \\ 7503.1285 & 7.15 \mathrm{E}-02 & 7502.9875 & 5.57 \mathrm{E}-02 \\ 7503.381 & 7.40 \mathrm{E}-02 & 7503.24 & 5.71 \mathrm{E}-02 \\ 7503.6285 & 7.61 \mathrm{E}-02 & 7503.4875 & 5.79 \mathrm{E}-02 \\ 7503.881 & 7.87 \mathrm{E}-02 & 7503.74 & 5.97 \mathrm{E}-02 \\ 7504.1285 & 8.15 \mathrm{E}-02 & 7503.9875 & 6.16 \mathrm{E}-02 \\ 7504.3815 & 8.42 \mathrm{E}-02 & 7504.2405 & 6.33 \mathrm{E}-02 \\ 7504.6285 & 8.70 \mathrm{E}-02 & 7504.4875 & 6.47 \mathrm{E}-02 \\ 7504.8815 & 8.99 \mathrm{E}-02 & 7504.7405 & 6.71 \mathrm{E}-02 \\ 7505.129 & 9.31 \mathrm{E}-02 & 7504.988 & 6.92 \mathrm{E}-02 \\ 7505.382 & 9.66 \mathrm{E}-02 & 7505.241 & 7.10 \mathrm{E}-02 \\ 7505.6295 & 0.10016511 & 7505.4885 & 7.32 \mathrm{E}-02 \\ 7505.8824 & 0.10385003 & 7505.7414 & 7.76 \mathrm{E}-02 \\ 7506.13 & 0.10792794 & 7505.989 & 8.00 \mathrm{E}-02 \\ 7506.3771 & 0.1120441 & 7506.2361 & 8.22 \mathrm{E}-02 \\ 7506.6305 & 0.11677193 & 7506.4895 & 8.45 \mathrm{E}-02 \\ 7506.878 & 0.12188348 & 7506.737 & 8.71 \mathrm{E}-02 \\ 7507.131 & 0.12690383 & 7506.99 & 9.03 \mathrm{E}-02 \\ 7507.3785 & 0.13172564 & 7507.2375 & 9.30 \mathrm{E}-02 \\ 7507.6315 & 0.13778136 & 7507.4905 & 9.66 \mathrm{E}-02 \\ 7507.879 & 0.14389869 & 7507.738 & 9.98 \mathrm{E}-02 \\ 7508.1324 & 0.1504027 & 7507.9914 & 0.1041356 \\ 7508.38 & 0.15725092 & 7508.239 & 0.10854797 \\ 7508.6276 & 0.16464096 & 7508.4866 & 0.11280535 \\ 7508.881 & 0.17373648 & 7508.74 & 0.11741849 \\ 7509.1285 & 0.18329261 & 7508.9875 & 0.12260055 \\ 7509.3815 & 0.19372481 & 7509.2405 & 0.1282532 \\ 7509.6295 & 0.20477011 & 7509.4885 & 0.13399226 \\ 7509.8824 & 0.2169477 & 7509.7414 & 0.14061587 \\ 7510.1305 & 0.2301936 & 7509.9895 & 0.14732811 \\ 7510.378 & 0.24398367 & 7510.237 & 0.1547655 \\ 7510.6315 & 0.25899366 & 7510.4905 & 0.16363626 \\ 7510.8795 & 0.27442305 & 7510.7385 & 0.17249556 \\ 7511.1329 & 0.29049903 & 7510.9919 & 0.18154182 \\ 7511.3805 & 0.30648521 & 7511.2395 & 0.19170033 \\ 7511.6285 & 0.32242349 & 7511.4875 & 0.2024197 \\ 7511.882 & 0.3391277 & 7511.741 & 0.21347595 \\ 7512.631 & 0.39217906 & 7512.49 & 0.24762862\end{array}$




$\begin{array}{rrrr}7512.879 & 0.4127516 & 7512.738 & 0.25972517 \\ 7513.1324 & 0.43703399 & 7512.9914 & 0.27276108 \\ 7513.3805 & 0.46298442 & 7513.2395 & 0.2869753 \\ 7513.6285 & 0.49264853 & 7513.4875 & 0.30055441 \\ 7513.8824 & 0.52837159 & 7513.7414 & 0.31526914 \\ 7514.1305 & 0.567739 & 7513.9895 & 0.330348 \\ 7514.3785 & 0.61150436 & 7514.2375 & 0.3480076 \\ 7514.632 & 0.66443964 & 7514.491 & 0.36791968 \\ 7514.88 & 0.72236581 & 7514.739 & 0.38947341 \\ 7515.128 & 0.78851523 & 7514.987 & 0.41484755 \\ 7515.382 & 0.8657957 & 7515.241 & 0.44303948 \\ 7515.63 & 0.95016461 & 7515.489 & 0.47589151 \\ 7515.878 & 1.0442672 & 7515.737 & 0.51447021 \\ 7516.132 & 1.152439 & 7515.991 & 0.55850932 \\ 7516.38 & 1.2672395 & 7516.239 & 0.6091655 \\ 7516.6285 & 1.3898238 & 7516.4875 & 0.66585983 \\ 7516.8824 & 1.5217756 & 7516.7414 & 0.72947445 \\ 7517.1305 & 1.6538792 & 7516.9895 & 0.79785008 \\ 7517.379 & 1.78632 & 7517.238 & 0.87223466 \\ 7517.6324 & 1.9154792 & 7517.4914 & 0.95163801 \\ 7517.881 & 2.0308221 & 7517.74 & 1.0314846 \\ 7518.1295 & 2.1341045 & 7517.9885 & 1.111066 \\ 7518.3776 & 2.2184694 & 7518.2366 & 1.1887743 \\ 7518.6315 & 2.285376 & 7518.4905 & 1.2629806 \\ 7518.88 & 2.3288549 & 7518.739 & 1.329522 \\ 7519.1285 & 2.3501961 & 7518.9875 & 1.3890638 \\ 7519.3824 & 2.3549279 & 7519.2414 & 1.4412091 \\ 7519.631 & 2.3393706 & 7519.49 & 1.48258 \\ 7519.8795 & 2.3123253 & 7519.7385 & 1.5147959 \\ 7520.128 & 2.2758619 & 7519.987 & 1.5382799 \\ 7520.382 & 2.2333428 & 7520.241 & 1.5562866 \\ 7520.6305 & 2.1887194 & 7520.4895 & 1.5673747 \\ 7520.879 & 2.1435499 & 7520.738 & 1.5720363 \\ 7521.1276 & 2.1006304 & 7520.9866 & 1.5710504 \\ 7521.382 & 2.0582416 & 7521.241 & 1.5643735 \\ 7521.6305 & 2.017911 & 7521.4895 & 1.5547881 \\ 7521.879 & 1.97905 & 7521.738 & 1.5428819 \\ 7522.1276 & 1.940704 & 7521.9866 & 1.5310066 \\ 7522.382 & 1.9015424 & 7522.241 & 1.5182631 \\ 7522.6305 & 1.8620236 & 7522.4895 & 1.5081118\end{array}$




$\begin{array}{rrrr}7522.879 & 1.8220044 & 7522.738 & 1.4981079 \\ 7523.128 & 1.7836277 & 7522.987 & 1.4906687 \\ 7523.3824 & 1.744184 & 7523.2414 & 1.4837692 \\ 7523.631 & 1.7032747 & 7523.49 & 1.4793667 \\ 7523.88 & 1.6629709 & 7523.739 & 1.478018 \\ 7524.1285 & 1.6233613 & 7523.9875 & 1.478475 \\ 7524.3776 & 1.5845661 & 7524.2366 & 1.4831657 \\ 7524.632 & 1.5460828 & 7524.491 & 1.4902322 \\ 7524.8805 & 1.510463 & 7524.7395 & 1.4986708 \\ 7525.1295 & 1.4750324 & 7524.9885 & 1.5088418 \\ 7525.3785 & 1.4436111 & 7525.2375 & 1.5264337 \\ 7525.6271 & 1.4119165 & 7525.4861 & 1.5442117 \\ 7525.882 & 1.3816971 & 7525.741 & 1.5689516 \\ 7526.1305 & 1.3543147 & 7525.9895 & 1.5975397 \\ 7526.3795 & 1.3283151 & 7526.2385 & 1.6314046 \\ 7526.6285 & 1.3037113 & 7526.4875 & 1.6702004 \\ 7526.8776 & 1.2790105 & 7526.7366 & 1.7119291 \\ 7527.132 & 1.2570327 & 7526.991 & 1.7559676 \\ 7527.381 & 1.236728 & 7527.24 & 1.7996295 \\ 7527.6305 & 1.2169782 & 7527.4895 & 1.8424082 \\ 7527.8795 & 1.197893 & 7527.7385 & 1.8792241 \\ 7528.1285 & 1.179987 & 7527.9875 & 1.9067655 \\ 7528.3776 & 1.1629217 & 7528.2366 & 1.9247427 \\ 7528.6324 & 1.1468984 & 7528.4914 & 1.929716 \\ 7528.8815 & 1.1304773 & 7528.7405 & 1.918385 \\ 7529.1305 & 1.1150176 & 7528.9895 & 1.8930509 \\ 7529.3795 & 1.1003308 & 7529.2385 & 1.8536428 \\ 7529.629 & 1.0863778 & 7529.488 & 1.8052595 \\ 7529.878 & 1.0736138 & 7529.737 & 1.7498789 \\ 7530.1276 & 1.0612633 & 7529.9866 & 1.694442 \\ 7530.382 & 1.0484457 & 7530.241 & 1.6376631 \\ 7530.6315 & 1.0371055 & 7530.4905 & 1.5870466 \\ 7530.881 & 1.026414 & 7530.74 & 1.5441202 \\ 7531.13 & 1.016016 & 7530.989 & 1.5033825 \\ 7531.3795 & 1.0060919 & 7531.2385 & 1.4706738 \\ 7531.878 & 0.98679443 & 7531.737 & 1.4169097 \\ 752.632 & 0.96308831 & 7532.491 & 1.3568296\end{array}$




$\begin{array}{rrrr}7532.8815 & 0.95616081 & 7532.7405 & 1.3396902 \\ 7533.131 & 0.95048532 & 7532.99 & 1.3232153 \\ 7533.38 & 0.94445787 & 7533.239 & 1.3054698 \\ 7533.6295 & 0.9388672 & 7533.4885 & 1.2886098 \\ 7533.879 & 0.93386543 & 7533.738 & 1.2719593 \\ 7534.129 & 0.92905178 & 7533.988 & 1.2558838 \\ 7534.3785 & 0.92487674 & 7534.2375 & 1.2410842 \\ 7534.628 & 0.92168483 & 7534.487 & 1.2252947 \\ 7534.8776 & 0.91817525 & 7534.7366 & 1.2098889 \\ 7535.1324 & 0.91463408 & 7534.9914 & 1.1934409 \\ 7535.3824 & 0.91176194 & 7535.2414 & 1.1788221 \\ 7535.632 & 0.90872727 & 7535.491 & 1.1643961 \\ 7535.8815 & 0.90573249 & 7535.7405 & 1.1508937 \\ 7536.1315 & 0.90299991 & 7535.9905 & 1.138393 \\ 7536.381 & 0.9006713 & 7536.24 & 1.1241691 \\ 7536.6305 & 0.89855916 & 7536.4895 & 1.1110538 \\ 7536.8805 & 0.89643309 & 7536.7395 & 1.0990948 \\ 7537.13 & 0.89401955 & 7536.989 & 1.0858838 \\ 7537.38 & 0.89198966 & 7537.239 & 1.0743854 \\ 7537.6295 & 0.89062247 & 7537.4885 & 1.0636911 \\ 7537.8795 & 0.888784 & 7537.7385 & 1.0527602 \\ 7538.1295 & 0.88735158 & 7537.9885 & 1.0429445 \\ 7538.379 & 0.88647644 & 7538.238 & 1.0342546 \\ 7538.629 & 0.88520419 & 7538.488 & 1.0240654 \\ 7538.879 & 0.88534879 & 7538.738 & 1.0156745 \\ 7539.129 & 0.88645951 & 7538.988 & 1.0073917 \\ 7539.379 & 0.88680986 & 7539.238 & 0.99987066 \\ 7539.6285 & 0.88765218 & 7539.4875 & 0.994004 \\ 7539.8785 & 0.88803133 & 7539.7375 & 0.98845479 \\ 7540.1285 & 0.88801937 & 7539.9875 & 0.98164605 \\ 7540.3785 & 0.8892012 & 7540.2375 & 0.9768121 \\ 7540.6285 & 0.89082246 & 7540.4875 & 0.97262984 \\ 7540.8785 & 0.89303067 & 7540.7375 & 0.96838803 \\ 7541.1285 & 0.89541996 & 7540.9875 & 0.963341 \\ 7541.379 & 0.89815992 & 7541.238 & 0.96057032 \\ 7541.629 & 0.90134226 & 7541.488 & 0.95558558 \\ 7541.879 & 0.90450358 & 7541.738 & 0.95139536 \\ 7542.129 & 0.9081021 & 7541.988 & 0.94784121 \\ 7542.3795 & 0.91124096 & 7542.2385 & 0.9455671 \\ 7542.6295 & 0.91444369 & 7542.4885 & 0.94311949\end{array}$




$\begin{array}{rrrr}7542.8795 & 0.9181262 & 7542.7385 & 0.94032311 \\ 7543.13 & 0.92194317 & 7542.989 & 0.93786816 \\ 7543.38 & 0.92642126 & 7543.239 & 0.93535338 \\ 7543.6305 & 0.92978001 & 7543.4895 & 0.93253843 \\ 7543.8805 & 0.93237197 & 7543.7395 & 0.93099297 \\ 7544.1808 & 0.93705057 & 7544.0398 & 0.92990824 \\ 7544.4811 & 0.94107415 & 7544.3401 & 0.92905139 \\ 7544.7814 & 0.9461022 & 7544.6404 & 0.92722155 \\ 7545.0821 & 0.95024127 & 7544.9411 & 0.92608236 \\ 7545.3824 & 0.9542154 & 7545.2414 & 0.92521713 \\ 7545.6774 & 0.95764427 & 7545.5364 & 0.92486139 \\ 7545.9777 & 0.96165504 & 7545.8367 & 0.92305798 \\ 7546.2784 & 0.96475819 & 7546.1374 & 0.922885 \\ 7546.5787 & 0.96699856 & 7546.4377 & 0.92402598 \\ 7546.8795 & 0.96892111 & 7546.7385 & 0.92384781 \\ 7547.1798 & 0.97115613 & 7547.0388 & 0.92493173 \\ 7547.4806 & 0.9722775 & 7547.3396 & 0.92461435 \\ 7547.7814 & 0.97388368 & 7547.6404 & 0.92484193 \\ 7548.0821 & 0.97488081 & 7547.9411 & 0.92623044 \\ 7548.3829 & 0.97647871 & 7548.2419 & 0.92599016 \\ 7548.6779 & 0.97685065 & 7548.5369 & 0.92708795 \\ 7548.9786 & 0.97633292 & 7548.8376 & 0.9300079 \\ 7549.2794 & 0.97712991 & 7549.1384 & 0.93111433 \\ 7549.5802 & 0.97789728 & 7549.4392 & 0.93359755 \\ 7549.881 & 0.97881399 & 7549.74 & 0.93488061 \\ 7550.1818 & 0.97964777 & 7550.0408 & 0.9371038 \\ 7550.4825 & 0.98002694 & 7550.3415 & 0.93817548 \\ 7550.7779 & 0.98159517 & 7550.6369 & 0.9405312 \\ 7551.0792 & 0.9822663 & 7550.9382 & 0.9421978 \\ 7551.38 & 0.98331319 & 7551.239 & 0.94348397 \\ 7551.6808 & 0.98507751 & 7551.5398 & 0.94512611 \\ 7551.9821 & 0.9861175 & 7551.8411 & 0.94618292 \\ 7552.2775 & 0.98698039 & 7552.1365 & 0.94917082 \\ 7552.5787 & 0.9893145 & 7552.4377 & 0.9508087 \\ 7552.8795 & 0.99146103 & 7552.7385 & 0.95142794 \\ 7553.1808 & 0.9933864 & 7553.0398 & 0.95452356 \\ 7553.4821 & 0.99476999 & 7553.3411 & 0.95572988 \\ 7553.7775 & 0.99699573 & 7553.6365 & 0.95761809 \\ 7554.0787 & 0.99875058 & 7553.9377 & 0.95941306 \\ 7554.38 & 1.0009464 & 7554.239 & 0.96148904\end{array}$




$\begin{array}{rrrr}7554.6813 & 1.0026158 & 7554.5403 & 0.9624912 \\ 7554.9825 & 1.0048647 & 7554.8415 & 0.96354208 \\ 7555.2784 & 1.0066089 & 7555.1374 & 0.96534373 \\ 7555.5797 & 1.0084886 & 7555.4387 & 0.96693469 \\ 7555.881 & 1.0096849 & 7555.74 & 0.9693086 \\ 7556.1822 & 1.0113803 & 7556.0412 & 0.97113661 \\ 7556.4781 & 1.0134336 & 7556.3371 & 0.97243784 \\ 7556.7794 & 1.0153083 & 7556.6384 & 0.97528296 \\ 7557.0812 & 1.0175404 & 7556.9402 & 0.97663227 \\ 7557.3824 & 1.019635 & 7557.2414 & 0.9792617 \\ 7557.6783 & 1.0215356 & 7557.5373 & 0.98084955 \\ 7557.9801 & 1.0239416 & 7557.8391 & 0.9827834 \\ 7558.2819 & 1.0257927 & 7558.1409 & 0.98414326 \\ 7558.5778 & 1.0270363 & 7558.4368 & 0.98512331 \\ 7558.8795 & 1.0281078 & 7558.7385 & 0.98608063 \\ 7559.1808 & 1.0300295 & 7559.0398 & 0.98769313 \\ 7559.4825 & 1.0314756 & 7559.3415 & 0.98808973 \\ 7559.7789 & 1.032576 & 7559.6379 & 0.9888516 \\ 7560.0807 & 1.0340248 & 7559.9397 & 0.99033828 \\ 7560.3824 & 1.0346707 & 7560.2414 & 0.98998085 \\ 7560.6783 & 1.0358041 & 7560.5373 & 0.98938985 \\ 7560.9801 & 1.0369476 & 7560.8391 & 0.99095351 \\ 7561.2823 & 1.0381016 & 7561.1413 & 0.99110088 \\ 7561.5782 & 1.0394429 & 7561.4372 & 0.99035398 \\ 7561.8805 & 1.0397018 & 7561.7395 & 0.9906272 \\ 7562.1822 & 1.0397269 & 7562.0412 & 0.99114572 \\ 7562.4786 & 1.040295 & 7562.3376 & 0.99052546 \\ 7562.7804 & 1.0406457 & 7562.6394 & 0.99042713 \\ 7563.0826 & 1.0420767 & 7562.9416 & 0.99022591 \\ 7563.379 & 1.0428919 & 7563.238 & 0.98957441 \\ 7563.6813 & 1.0431153 & 7563.5403 & 0.9895484 \\ 7563.9777 & 1.0434652 & 7563.8367 & 0.98902359 \\ 7564.2799 & 1.0434708 & 7564.1389 & 0.98744892 \\ 7564.5817 & 1.0437476 & 7564.4407 & 0.98812896 \\ 7564.8785 & 1.0432396 & 7564.7375 & 0.98713276 \\ 7565.1808 & 1.044787 & 7565.0398 & 0.98753723 \\ 7565.483 & 1.0440526 & 7565.342 & 0.98672312 \\ 7565.7794 & 1.0439785 & 7565.6384 & 0.98646077 \\ 7566.0817 & 1.0436282 & 7565.9407 & 0.98711098 \\ 7566.3785 & 1.0442149 & 7566.2375 & 0.98704066\end{array}$




$\begin{array}{rrrr}7566.6808 & 1.044107 & 7566.5398 & 0.98745184 \\ 7566.9777 & 1.0442861 & 7566.8367 & 0.98771624 \\ 7567.2799 & 1.0440211 & 7567.1389 & 0.98893119 \\ 7567.5821 & 1.0439036 & 7567.4411 & 0.99031754 \\ 7567.879 & 1.0436274 & 7567.738 & 0.99087724 \\ 7568.1818 & 1.0438998 & 7568.0408 & 0.99108927 \\ 7568.4786 & 1.0439253 & 7568.3376 & 0.99195244 \\ 7568.7809 & 1.0429651 & 7568.6399 & 0.99184489 \\ 7569.0778 & 1.0435849 & 7568.9368 & 0.9923616 \\ 7569.3805 & 1.0439428 & 7569.2395 & 0.99435712 \\ 7569.6774 & 1.0420764 & 7569.5364 & 0.99517398 \\ 7569.9801 & 1.0418393 & 7569.8391 & 0.99598201 \\ 7570.2828 & 1.0411768 & 7570.1418 & 0.99584712 \\ 7570.5797 & 1.0403084 & 7570.4387 & 0.99783279 \\ 7570.8824 & 1.0394068 & 7570.7414 & 0.99826545 \\ 7571.1798 & 1.0381429 & 7571.0388 & 0.9992906 \\ 7571.4825 & 1.0366734 & 7571.3415 & 1.0000697 \\ 7571.7794 & 1.0358043 & 7571.6384 & 1.0011861 \\ 7572.0821 & 1.0344424 & 7571.9411 & 1.0013443 \\ 7572.3795 & 1.0333023 & 7572.2385 & 1.0031119 \\ 7572.6822 & 1.0323749 & 7572.5412 & 1.0041975 \\ 7572.9796 & 1.0302042 & 7572.8386 & 1.0065304 \\ 7573.2823 & 1.028535 & 7573.1413 & 1.0091908 \\ 7573.5797 & 1.0272471 & 7573.4387 & 1.0111048 \\ 7573.8771 & 1.0257616 & 7573.7361 & 1.0127488 \\ 7574.1803 & 1.024556 & 7574.0393 & 1.0142938 \\ 7574.4777 & 1.0224639 & 7574.3367 & 1.0156735 \\ 7574.7809 & 1.0206998 & 7574.6399 & 1.0172937 \\ 7575.0782 & 1.0184977 & 7574.9372 & 1.0191977 \\ 7575.381 & 1.0162536 & 7575.24 & 1.0198867 \\ 7575.6788 & 1.0148073 & 7575.5378 & 1.0225209 \\ 7575.9821 & 1.0123015 & 7575.8411 & 1.0233094 \\ 7576.2794 & 1.0103891 & 7576.1384 & 1.0246991 \\ 7576.5826 & 1.008262 & 7576.4416 & 1.0257009 \\ 7576.8805 & 1.0062556 & 7576.7395 & 1.0261979 \\ 7577.1779 & 1.003578 & 7577.0369 & 1.028132 \\ 7577.4811 & 1.0009007 & 7577.3401 & 1.0283839 \\ 7577.7789 & 0.99858655 & 7577.6379 & 1.0295651 \\ 7578.38 & 0.99565839 & 7578.239 & 1.0304641\end{array}$




$\begin{array}{rrrr}7578.6779 & 0.99353144 & 7578.5369 & 1.031576 \\ 7578.9811 & 0.99084385 & 7578.8401 & 1.0322799 \\ 7579.2789 & 0.98981138 & 7579.1379 & 1.0316237 \\ 7579.5826 & 0.98778912 & 7579.4416 & 1.0321234 \\ 7579.8805 & 0.98627235 & 7579.7395 & 1.0320824 \\ 7580.1783 & 0.98425426 & 7580.0373 & 1.0334135 \\ 7580.4816 & 0.98192402 & 7580.3406 & 1.0343963 \\ 7580.7799 & 0.98007454 & 7580.6389 & 1.0344531 \\ 7581.0778 & 0.9790635 & 7580.9368 & 1.0330554 \\ 7581.3815 & 0.9777126 & 7581.2405 & 1.0328999 \\ 7581.6793 & 0.97556058 & 7581.5383 & 1.0324836 \\ 7581.9777 & 0.9739666 & 7581.8367 & 1.0323362 \\ 7582.2814 & 0.97258473 & 7582.1404 & 1.0328708 \\ 7582.5792 & 0.97042002 & 7582.4382 & 1.0311388 \\ 7582.8776 & 0.9686069 & 7582.7366 & 1.0306695 \\ 7583.1813 & 0.96647464 & 7583.0403 & 1.0297246 \\ 7583.4791 & 0.96432214 & 7583.3381 & 1.0286314 \\ 7583.7775 & 0.96295962 & 7583.6365 & 1.0268879 \\ 7583.879 & 0.96220752 & 7583.738 & 1.0251051 \\ 7586.8795 & 0.94929252 & 7586.7385 & 1.0142837 \\ 7589.8824 & 0.9430213 & 7589.7414 & 1.0005576 \\ 7592.8771 & 0.94377819 & 7592.7361 & 0.98599938 \\ 7595.88 & 0.95316537 & 7595.739 & 0.97468168 \\ 7598.88 & 0.96995796 & 7598.739 & 0.9695277 \\ 7601.8824 & 0.99043035 & 7601.7414 & 0.96732475 \\ 7604.882 & 1.0088139 & 7604.741 & 0.96936374 \\ 7607.878 & 1.0207365 & 7607.737 & 0.97449185 \\ 7610.8771 & 1.0248177 & 7610.7361 & 0.97976657 \\ 7613.879 & 1.0229164 & 7613.738 & 0.98533739 \\ 7616.8776 & 1.0169858 & 7616.7366 & 0.99093219 \\ 7619.8785 & 1.0093078 & 7619.7375 & 0.99637947 \\ 7622.8824 & 1.0042466 & 7622.7414 & 0.99896115 \\ 7625.8776 & 0.99959328 & 7625.7366 & 0.9993623 \\ 7628.881 & 0.99865943 & 7628.74 & 0.99879948 \\ 7631.8815 & 0.99993057 & 7631.7405 & 0.99784632 \\ 7634.8785 & 1.0009963 & 7634.7375 & 0.9963892 \\ 7637.8785 & 1.0029607 & 7637.7375 & 0.99409793 \\ 7640.881 & 1.0052532 & 7640.74 & 0.99420305 \\ 7643.88 & 1.0055443 & 7643.739 & 0.99639264 \\ 7646.882 & 1.0040485 & 7646.741 & 0.99948428\end{array}$




$\begin{array}{rrrr}7649.881 & 1.0008905 & 7649.74 & 1.0023974 \\ 7652.8824 & 0.99571656 & 7652.7414 & 1.0042431 \\ 7655.8805 & 0.99220426 & 7655.7395 & 1.0070493 \\ 7658.8815 & 0.98607904 & 7658.7405 & 1.0088895 \\ 7661.8795 & 0.98251586 & 7661.7385 & 1.0093054 \\ 7664.8795 & 0.97906421 & 7664.7385 & 1.0089872 \\ 7667.8829 & 0.97943997 & 7667.7419 & 1.008506 \\ 7670.8824 & 0.9810659 & 7670.7414 & 1.0048496 \\ 7673.879 & 0.9831566 & 7673.738 & 1.0023478 \\ 7676.8785 & 0.9871666 & 7676.7375 & 0.9986941 \\ 7679.88 & 0.99136303 & 7679.739 & 0.99398361 \\ 7682.8785 & 0.9969684 & 7682.7375 & 0.98862868 \\ 7685.88 & 1.0042949 & 7685.739 & 0.98455648 \\ 7688.878 & 1.0096051 & 7688.737 & 0.98322102 \\ 7691.8785 & 1.0139013 & 7691.7375 & 0.98377264 \\ 7694.882 & 1.0164343 & 7694.741 & 0.98562834 \\ 7697.882 & 1.0169004 & 7697.741 & 0.98836796 \\ 7700.8785 & 1.0156125 & 7700.7375 & 0.99216435 \\ 7703.878 & 1.012999 & 7703.737 & 0.99805353 \\ 7706.88 & 1.0098877 & 7706.739 & 1.0021028 \\ 7709.8785 & 1.0050824 & 7709.7375 & 1.0059935 \\ 7712.8795 & 1.0017944 & 7712.7385 & 1.0076026 \\ 7715.8776 & 0.99916699 & 7715.7366 & 1.0081792 \\ 7718.878 & 0.99681931 & 7718.737 & 1.0072188 \\ 7721.8815 & 0.99577727 & 7721.7405 & 1.0060874 \\ 7724.8815 & 0.99693938 & 7724.7405 & 1.0055544 \\ 7727.878 & 0.99801872 & 7727.737 & 1.0035093 \\ 7730.8829 & 1.0001259 & 7730.7419 & 1.0020947 \\ 7733.8785 & 1.0017632 & 7733.7375 & 1.0015461 \\ 7736.8771 & 1.0033517 & 7736.7361 & 1.0007281 \\ 7739.878 & 1.0048569 & 7739.737 & 0.99940022 \\ 7742.882 & 1.0060323 & 7742.741 & 1.0004599 \\ 7745.882 & 1.0063572 & 7745.741 & 1.0012938 \\ 7748.879 & 1.005461 & 7748.738 & 1.0020457 \\ 7754.8805 & 1.0025569 & 7754.7395 & 1.0037886 \\ 7757.879 & 1.000308 & 7757.738 & 1.0056596 \\ 7766.879 & 0.99622857 & 7766.738 & 1.0078874\end{array}$




$\begin{array}{rrrr}7769.882 & 0.99569474 & 7769.741 & 1.0082497 \\ 7772.8815 & 0.9952441 & 7772.7405 & 1.0071455 \\ 7775.878 & 0.99549392 & 7775.737 & 1.0039343 \\ 7778.8824 & 0.99544367 & 7778.7414 & 1.0026313 \\ 7781.878 & 0.99561124 & 7781.737 & 0.99926025 \\ 7784.882 & 0.99746538 & 7784.741 & 0.99785774 \\ 7787.8824 & 0.9998162 & 7787.7414 & 0.9958213 \\ 7790.8795 & 1.0015968 & 7790.7385 & 0.99477349 \\ 7793.879 & 1.0036103 & 7793.738 & 0.99345683 \\ 7796.8815 & 1.0055887 & 7796.7405 & 0.99222738 \\ 7799.88 & 1.0066781 & 7799.739 & 0.99140566 \\ 7802.8815 & 1.0080855 & 7802.7405 & 0.9909589 \\ 7805.8795 & 1.0077103 & 7805.7385 & 0.99221035 \\ 7808.88 & 1.0074915 & 7808.739 & 0.99277038 \\ 7811.8829 & 1.0057753 & 7811.7419 & 0.9929021 \\ 7814.8824 & 1.0047209 & 7814.7414 & 0.99440182 \\ 7817.8785 & 1.0023955 & 7817.7375 & 0.99612765 \\ 7820.8829 & 0.99985395 & 7820.7419 & 0.99408239 \\ 7823.878 & 0.99832155 & 7823.737 & 0.99665632 \\ 7826.8815 & 0.99647537 & 7826.7405 & 0.99775597 \\ 7829.8815 & 0.9946095 & 7829.7405 & 0.99982917 \\ 7832.8785 & 0.99268542 & 7832.7375 & 0.99935692 \\ 7835.8776 & 0.99066683 & 7835.7366 & 0.99925242 \\ 7838.879 & 0.99011772 & 7838.738 & 0.99954224 \\ 7841.8771 & 0.99167916 & 7841.7361 & 0.99809218 \\ 7844.878 & 0.99004968 & 7844.737 & 0.99644937 \\ 7847.8815 & 0.99077638 & 7847.7405 & 0.99420839 \\ 7850.881 & 0.99102906 & 7850.74 & 0.99429148 \\ 7853.8771 & 0.99079883 & 7853.7361 & 0.99231181 \\ 7856.882 & 0.99338686 & 7856.741 & 0.99160576 \\ 7859.8829 & 0.99279659 & 7859.7419 & 0.98899501 \\ 7862.8805 & 0.99346395 & 7862.7395 & 0.98944143 \\ 7865.8805 & 0.99400957 & 7865.7395 & 0.98923994\end{array}$




\section{References}

1. Krzystek, J.; Fiedler, A. T.; Sokol, J. J.; Ozarowski, A.; Zvyagin, S. A.; Brunold, T. C.; Long, J. R.; Brunel, L.-C.; Telser, J., Inorganic Chemistry 2004, 43(18), 5645.

2. Chilton, N. F.; Anderson, R. P.; Turner, L. D.; Soncini, A.; Murray, K. S., Journal of Computational Chemistry 2013, 34(13), 1164.

3. Ginsberg, A. P.; Martin, R. L.; Brookes, R. W.; Sherwood, R. C., Magnetic exchange in transition metal complexes. IX. Dimeric nickel(II)-ethylenediamine complexes.

Inorganic Chemistry 1972, 11 (12), 2884-2889. 